PARAMETRIC PROPERTIES OF SEMI-NONPARAMETRIC DISTRIBUTIONS. WITH APPLICATIONS TO OPTION VALUATION

Angel león, Javier Mencia and Enrique Sentana.

Documentos de Trabajo N. 0707

\title{
BATCOD ESPANA
}

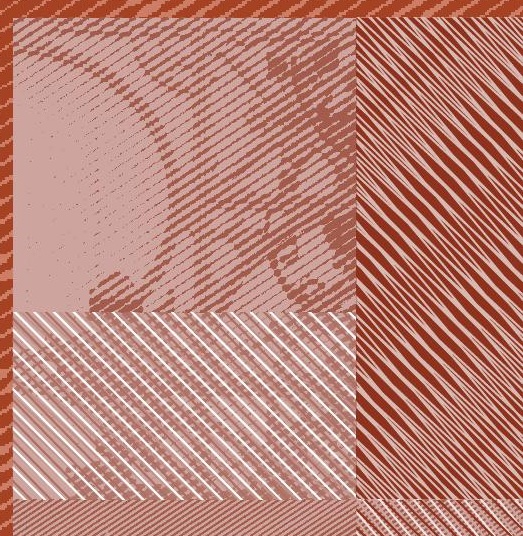


PARAMETRIC PROPERTIES OF SEMI-NONPARAMETRIC DISTRIBUTIONS,

WITH APPLICATIONS TO OPTION VALUATION 
PARAMETRIC PROPERTIES OF SEMI-NONPARAMETRIC

DISTRIBUTIONS, WITH APPLICATIONS TO OPTION VALUATION $\left.{ }^{*}\right)$

\author{
Ángel León \\ UNIVERSIDAD DE ALICANTE \\ Javier Mencía \\ BANCO DE ESPAÑA \\ Enrique Sentana \\ CEMFI
}

${ }^{*}$ ) We are grateful to Peter Carr, Eva Ferreira, René Garcia, Vance Martin, Nour Meddahi, Antonio Mele, Michael Rockinger, George Tauchen and Fernando Zapatero, as well as seminar audiences at CREST, EFMA (Madrid, 2006), LSE FMG, the CIRANO-CIREQ Financial Econometrics Conference (Montreal, 2006), the Econometric Society World Congress (London, 2005), the Finance Forum (Barcelona, 2004), and the Symposium on Economic Analysis (Pamplona, 2004). We are also extremely thankful to Bernard Dumas for allowing us to use his database in the empirical application. Of course, the usual caveat applies. The views expressed in this paper are those of the authors, and do not reflect the views of the Bank of Spain. Financial support from Fundación Ramón Areces (Mencía) and the Spanish Ministry of Education and Science through the grants SEJ 2005-09372 (León) and SEJ 2005-08880 (Sentana) is gratefully acknowledged. Address for correspondence: Casado del Alisal 5, 28014 Madrid, Spain, tel.: +34 914290551 , fax: +34 914291056 . 
The Working Paper Series seeks to disseminate original research in economics and finance. All papers have been anonymously refereed. By publishing these papers, the Banco de España aims to contribute to economic analysis and, in particular, to knowledge of the Spanish economy and its international environment.

The opinions and analyses in the Working Paper Series are the responsibility of the authors and, therefore, do not necessarily coincide with those of the Banco de España or the Eurosystem.

The Banco de España disseminates its main reports and most of its publications via the INTERNET at the following website: http://www.bde.es.

Reproduction for educational and non-commercial purposes is permitted provided that the source is acknowledged.

\section{(C) BANCO DE ESPAÑA, Madrid, 2007}

ISSN: 0213-2710 (print)

ISSN: 1579-8666 (on line)

Depósito legal: M.16177-2007

Imprenta del Banco de España 


\section{Abstract}

We derive the statistical properties of the SNP densities of Gallant and Nychka (1987). We show that these densities, which are always positive, are more flexible than truncated GramCharlier expansions with positivity restrictions. We use the SNP densities for financial derivatives valuation. We relate real and risk-neutral measures, obtain closed-form prices for European options, and analyse the semiparametric properties of our pricing model. In an empirical application to S\&P500 index options, we compare our model to the standard and Practitioner's Black-Scholes formulas, truncated expansions, and the Generalised Beta and Variance Gamma models.

Keywords: Kurtosis, Density Expansions, Gram-Charlier, Skewness, S\&P index options.

JEL: G13, C16. 


\section{Introduction}

In recent years, many studies have attempted to overcome the limitations of the popular normality assumption on the returns of stocks and other financial assets, which is often rejected in the empirical finance literature even after controlling for volatility clustering effects. Although this assumption may still be reasonable if the interest focuses on the first two conditional moments (see Bollerslev and Wooldridge, 1992), in many financial applications the features under study involve higher order moments such as skewness and kurtosis. An important example is option pricing theory, where the Black and Scholes (1973) pricing formula, which remains the benchmark model because of its analytical tractability, also relies on the normality of returns. Unfortunately, normality is too restrictive to approximate the complex shape of the distribution of most underlying asset returns, and more flexible distributions may help to explain the puzzles unresolved by the Black and Scholes (1973) framework, including smiles and smirks.

However, any successful generalisation of the Gaussian assumption must satisfy two crucial requirements: modelling flexibility and analytical tractability. Both needs are satisfied by Gram-Charlier expansions, which were introduced in option pricing theory by Jarrow and Rudd (1982), and have been used more recently by Corrado and Su (1996, 1997), Capelle-Blanchard, Jurczenko, and Maillet (2001), and Jurczenko, Maillet, and Negrea (2002a). As is well known, many density functions can be expressed as a possibly infinite expansion of the Gaussian density. In practice, however, the expansion is usually truncated after the fourth power. Unfortunately, such truncated expansions often imply negative densities over some interval of their domain of variation, as Jondeau and Rockinger (2001) emphasize. This feature is particularly worrying in option pricing applications because it allows some arbitrage opportunities. For instance, the price of a butterfly spread with positive payoff over an interval of negative density would necessarily be negative in those circumstances. As a solution to this problem, Jondeau and Rockinger (2001) propose to restrict the parameters of the expansion so that the density always remains positive. Unfortunately, their approach is difficult to implement even when the truncation order is low.

In this context, we propose the use of semi-nonparametric distributions (SNP), which were introduced by Gallant and Nychka (1987) for nonparametric estimation purposes, as 
an equally flexible and analytically tractable solution. The SNP density can be regarded as an alternative expansion of the Gaussian density function, which is always positive by construction.

The properties of the SNP density from the nonparametric estimation point of view have been studied in depth by Fenton and Gallant (1996) and Gallant and Tauchen (1999) for density estimation and in the implementation of the Efficient Method of Moments, respectively. However, this density has not been treated from a purely parametric point of view, that is, taking the SNP distribution as if it reflected the actual data generating process instead of an approximating kernel. In this sense, we assume that under the real measure asset returns follow a SNP distribution conditional on the information available at each point in time. In this framework, we will study first the statistical properties of this distribution, including moments, standardised versions, distribution of linear combinations, as well as its relationship to the Gram-Charlier densities. Then, we will combine it with an exponentially affine assumption on the stochastic discount factor, which will enable us to transform the real measure into the risk neutral measure required for the valuation of derivative assets. We will obtain closed-form expressions for European option prices by exploiting the analytical tractability of the SNP distribution. We will also compare the SNP with two other popular distributions in the option pricing literature: the Generalised Beta (GB), proposed by Bookstaber and McDonald (1987), and applied to option pricing by Liu et al. (2006) among others; and the Variance Gamma (VG) model of Madan and Milne (1991) and Madan, Carr, and Chang (1998). In addition, we will use the Marron and Wand (1992) test suite to assess the semiparametric properties of our option pricing model when the true model is not SNP. We also assess the ability of our model to fit the low frequency smiles generated by a high frequency SNP process with stochastic volatility. Furthermore, we will carry out an empirical application to the S\&P 500 options data of Dumas, Fleming, and Whaley (1998), in which we will evaluate the performance of our pricing formulas. Finally, we provide a generalised version of the SNP distribution that nests the ordinary SNP and the positive Gram-Charlier expansions considered by Jondeau and Rockinger (2001) as particular cases.

The paper is structured as follows. In the next section, we study the statistical properties of SNP densities, and compare them with those of Gram-Charlier expansions. 
In section 3, we first relate the real and risk neutral measures, and then focus on pricing European options. Section 4 studies the semiparametric properties of our methodology, while section 5 presents the empirical application. Finally, in section 6 we present our generalised SNP density, followed by our conclusions in section 7. Proofs and auxiliary results can be found in appendices.

\section{Density definition}

We want to analyse the statistical properties of the affine transformation $z=a+b x$, when the density of $x$ belongs to the semi-nonparametric class introduced by Gallant and Nychka (1987). Specifically,

$$
f(x ; \boldsymbol{\nu})=\frac{\phi(x)}{\boldsymbol{\nu}^{\prime} \boldsymbol{\nu}}\left(\sum_{i=0}^{m} \nu_{i} H_{i}(x)\right)^{2}
$$

where $\boldsymbol{\nu}=\left(\nu_{0}, \nu_{1}, \cdots, \nu_{m}\right)^{\prime} \in \mathbb{R}^{m+1}, \phi(\cdot)$ denotes the probability density function (pdf) of a standard normal random variable, and $H_{i}(x)$ is the normalised Hermite polynomial of order $i$. These polynomials can be defined recursively for $i \geq 2$ as

$$
H_{i}(x)=\frac{x H_{i-1}(x)-\sqrt{i-1} H_{i-2}(x)}{\sqrt{i}},
$$

with initial conditions $H_{0}(x)=1$ and $H_{1}(x)=x$. Importantly, $\left\{H_{i}(x)\right\}_{i \in \mathbb{N}}$ constitutes an orthonormal basis with respect to the weighting function $\phi(x)$, as illustrated by the following condition:

$$
\int_{-\infty}^{+\infty} H_{i}(x) H_{j}(x) \phi(x) d x=\mathbf{1}(i=j)
$$

where $\mathbf{1}(\cdot)$ is the usual indicator function. The change of variable formula implies that the density function of $z$ will be

$$
g(z ; \boldsymbol{\nu}, a, b)=\frac{1}{b} \frac{1}{\boldsymbol{\nu}^{\prime} \boldsymbol{\nu}} \phi\left(\frac{z-a}{b}\right)\left[\sum_{i=0}^{m} \nu_{i} H_{i}\left(\frac{z-a}{b}\right)\right]^{2}
$$

where we could interpret $a$ as a location parameter and $b$ as a scale parameter. Note that both (1) and (3) are homogeneous of degree zero in $\boldsymbol{\nu}$, which implies that there is a scale indeterminacy that we must solve by imposing a single normalising restriction on these parameters, such as $\nu_{0}=1$, or preferably $\boldsymbol{\nu}^{\prime} \boldsymbol{\nu}=1$, which we can ensure by working with hyperspherical coordinates. ${ }^{1}$

\footnotetext{
${ }^{1}$ That is, $\nu_{0}=\cos \alpha_{1} ; \nu_{i}=\left(\prod_{k=1}^{i} \sin \alpha_{k}\right) \cos \alpha_{i+1}$ for $\quad 0<i \leq m-1 ;$ and $\nu_{m}=\prod_{k=1}^{m} \sin \alpha_{k}$, where $\alpha_{k} \in[0, \pi)$, for $1<k \leq m-1$, and $\alpha_{m} \in[0,2 \pi)$. 
If we expand the squared expression in (1), we can obtain the following result:

Proposition 1 Let $x$ be a SNP random variable with density $f(x ; \boldsymbol{\nu})$ given by (1). Then:

$$
f(x ; \boldsymbol{\nu})=\phi(x) \sum_{k=0}^{2 m} \gamma_{k}(\boldsymbol{\nu}) H_{k}(x)
$$

where $\gamma_{0}(\boldsymbol{\nu})=1$,

$$
\gamma_{k}(\boldsymbol{\nu})=\frac{\boldsymbol{\nu}^{\prime} A_{k} \boldsymbol{\nu}}{\boldsymbol{\nu}^{\prime} \boldsymbol{\nu}}, \quad k \geq 1
$$

and $A_{k}$ is a $(m+1) \times(m+1)$ symmetric matrix whose typical element is

$$
a_{i j, k}=\frac{(i ! j ! k !)^{1 / 2}}{\left(\frac{i+j-k}{2}\right) !\left(\frac{i+k-j}{2}\right) !\left(\frac{k+j-i}{2}\right) !}
$$

if $k \in \Gamma$ and zero otherwise, with

$$
\Gamma=\left\{k \in \mathbb{N}:|i-j| \leq k \leq i+j ; \quad \frac{i-j+k}{2} \in \mathbb{N}\right\}
$$

For instance, the values of $\gamma_{k}(\boldsymbol{\nu})$ when $m=2$ are:

$$
\begin{array}{cc}
\gamma_{1}(\boldsymbol{\nu})=2 \nu_{1}\left(\nu_{0}+\sqrt{2} \nu_{2}\right) / \boldsymbol{\nu}^{\prime} \boldsymbol{\nu}, & \gamma_{2}(\boldsymbol{\nu})=\sqrt{2}\left(\nu_{1}^{2}+2 \nu_{2}^{2}+\sqrt{2} \nu_{0} \nu_{2}\right) / \boldsymbol{\nu}^{\prime} \boldsymbol{\nu} \\
\gamma_{3}(\boldsymbol{\nu})=2 \sqrt{3} \nu_{1} \nu_{2} / \boldsymbol{\nu}^{\prime} \boldsymbol{\nu}, & \gamma_{4}(\boldsymbol{\nu})=\sqrt{6} \nu_{2}^{2} / \boldsymbol{\nu}^{\prime} \boldsymbol{\nu} .
\end{array}
$$

\section{$2.1 \quad$ Moments of $x$ and $z$}

The first four non-central moments of $x, \mu_{x}^{\prime}(k)$, can be obtained by using the relationship between the powers of $x$ and the Hermite polynomials:

$$
\begin{aligned}
& \mu_{x}^{\prime}(1) \equiv E_{f}(x)=E_{f}\left[H_{1}(x)\right] \\
& \mu_{x}^{\prime}(2) \equiv E_{f}\left(x^{2}\right)=\sqrt{2} E_{f}\left[H_{2}(x)\right]+1 \\
& \mu_{x}^{\prime}(3) \equiv E_{f}\left(x^{3}\right)=\sqrt{3 !} E_{f}\left[H_{3}(x)\right]+3 E_{f}\left[H_{1}(x)\right] \\
& \mu_{x}^{\prime}(4) \equiv E_{f}\left(x^{4}\right)=\sqrt{4 !} E_{f}\left[H_{4}(x)\right]+6 \sqrt{2} E_{f}\left[H_{2}(x)\right]+3
\end{aligned}
$$

where the operator $E_{f}[\cdot]$ takes the expectation of its argument with respect to the density function $f(x ; \boldsymbol{\nu})$ in $(1)$. Then, from the previous non-central moments, the corresponding central ones, $\mu_{x}(k)$, can be easily obtained (see e.g. Stuart and Ord, 1977). Finally, we can also compute the skewness and kurtosis coefficients, denoted by $s k$ and $k u$, respectively. But since $\mu_{x}^{\prime}(k)$ in (6) depends on $\left\{E_{f}\left[H_{i}(x)\right]\right\}_{i \in \mathbb{N}}$, we first need to find these moments:

Proposition 2 Let $x$ denote the SNP random variable $x$ with density function (1). Then, the expected value of the $k$-th order Hermite polynomial are given by:

$$
E_{f}\left[H_{k}(x)\right]=\gamma_{k}(\boldsymbol{\nu})
$$

if $k \leq 2 m$, and zero otherwise, where $\gamma_{k}(\boldsymbol{\nu})$ is defined in (5). 
On this basis, we can easily compute the first four non-centred moments of $x$ for the important special case of $m=2$ :

Lemma 1 If the density function of the random variable $x$ is given by (1) with $m=2$, then

$$
\begin{array}{cl}
\mu_{x}^{\prime}(1)=\frac{2 \nu_{1}}{\nu^{\prime} \boldsymbol{\nu}}\left(\nu_{0}+\sqrt{2} \nu_{2}\right), & \mu_{x}^{\prime}(2)=\frac{2}{\boldsymbol{\nu}^{\prime} \boldsymbol{\nu}}\left(\nu_{1}^{2}+2 \nu_{2}^{2}+\sqrt{2} \nu_{2} \nu_{0}\right)+1 \\
\mu_{x}^{\prime}(3)=\frac{6 \nu_{1}}{\boldsymbol{\nu}^{\prime} \boldsymbol{\nu}}\left(\nu_{0}+2 \sqrt{2} \nu_{2}\right), & \mu_{x}^{\prime}(4)=\frac{12}{\boldsymbol{\nu}^{\prime} \boldsymbol{\nu}}\left(\nu_{1}^{2}+3 \nu_{2}^{2}+\sqrt{2} \nu_{2} \nu_{0}\right)+3
\end{array}
$$

More generally, we can show that:

Proposition 3 The moment generating function of the $S N P$ density (1) is $E_{f}[\exp (t x)]=$ $\exp \left(t^{2} / 2\right) \Lambda(\boldsymbol{\nu}, t)$, while its characteristic function is $\psi_{S N P}(i t)=\exp \left(-t^{2} / 2\right) \Lambda(\boldsymbol{\nu}, i t)$, where

$$
\Lambda(\boldsymbol{\nu}, t)=\sum_{k=0}^{2 m} \gamma_{k}(\boldsymbol{\nu}) \frac{t^{k}}{\sqrt{k !}}
$$

$\gamma_{k}(\boldsymbol{\nu})$ is defined in (5), and $i$ is the usual imaginary unit.

Since $z$ is an affine transformation of $x$, it is trivial to find the non-central moments of $z, \mu_{z}^{\prime}(k)$, as a function of those of $x$. Specifically,

$$
\mu_{z}^{\prime}(n) \equiv E_{f}\left[(a+b x)^{n}\right]=\sum_{i=0}^{n}\left(\begin{array}{c}
n \\
i
\end{array}\right) a^{n-i} b^{i} \mu_{x}^{\prime}(i)
$$

In addition, we can always choose the location and dispersion coefficients $a$ and $b$ such that $z$ has zero mean and unit variance. In particular, if we denote by $z^{*}$ the standardised variable

$$
z^{*}=\frac{x-\mu_{x}^{\prime}(1)}{\sqrt{\mu_{x}(2)}},
$$

then its density function can be directly obtained from (3) with

$$
a(\boldsymbol{\nu})=-\mu_{x}^{\prime}(1) / \sqrt{\mu_{x}(2)}, \quad b(\boldsymbol{\nu})=1 / \sqrt{\mu_{x}(2)} .
$$

We can also use Proposition 3 to derive the distribution of linear combinations of SNP variables. In particular, we can show that the distribution of the sum of $n$ iid SNP variables of order $m$ can be expressed as a Gram-Charlier expansion of order $n m$ that is always positive by construction.

Proposition 4 Define $q=\sum_{k=1}^{n} p_{k} x_{k}$, where $\left\{x_{k}\right\}_{k=1, \cdots, n}$ are iid random variables whose distribution is a SNP of order $m$ with shape parameters $\boldsymbol{\nu}$. Then, the distribution of $q$ is a Gram-Charlier expansion of order $2 m n$ whose density function can be expressed as

$$
\varphi(q)=\frac{\phi\left(\frac{q}{\|\mathbf{p}\|}\right)}{\|\mathbf{p}\|} \sum_{j=0}^{2 m n} d_{j}(\boldsymbol{\nu}, \mathbf{p}) H_{j}\left(\frac{q}{\|\mathbf{p}\|}\right)
$$


where $\mathbf{p}=\left(p_{1}, \cdots, p_{n}\right)^{\prime},\|p\|=\sqrt{\sum_{k=1}^{n} p_{k}^{2}}$ and

$$
d_{j}(\boldsymbol{\nu}, \mathbf{p})=\left.\sqrt{j} \frac{d^{j}}{d x^{j}}\left\{\prod_{i=1}^{k}\left[\sum_{k=0}^{2 m} \frac{\gamma_{k}(\boldsymbol{\nu})}{\sqrt{k !}}\left(p_{i} x\right)^{k}\right]\right\}\right|_{x=0}
$$

We will exploit this property to analyse the effect of time aggregation on SNP returns.

\subsection{Gram-Charlier expansion of the semi-nonparametric den- sity}

Under certain regularity conditions (see e.g. Stuart and Ord, 1977, p. 234), a density function $h(y)$ can be expressed as the product of a standard normal density times an infinite series of Hermite polynomials:

$$
\begin{aligned}
h(y) & =\phi(y) \sum_{k=0}^{\infty} c_{k} H_{k}(y), \\
c_{k} & =\int_{-\infty}^{\infty} H_{k}(y) h(y) d y=E_{h}\left(H_{k}(y)\right) .
\end{aligned}
$$

This is the so-called Gram-Charlier series of Type A.

With this in mind, we will first determine the Gram-Charlier expansion of the SNP density of $z$, and then we will particularise it for the standardised random variable $z^{*}$ in (9). In the case of $z$, we will use the fact that, according to (3) and (4), its density can be written as

$$
g(z ; \boldsymbol{\nu}, a, b)=\frac{1}{b} \phi\left(\frac{z-a}{b}\right) \sum_{i=0}^{2 m} \gamma_{i}(\boldsymbol{\nu}) H_{i}\left(\frac{z-a}{b}\right),
$$

where $\gamma_{i}(\boldsymbol{\nu})$ is defined in (5). Then, if we compare (14) and (15), we can write $c_{k}$ for $z$ as

$$
c_{k}=\frac{1}{b} \sum_{i=0}^{2 m} \gamma_{i}(\boldsymbol{\nu}) \int_{-\infty}^{\infty} \phi\left(\frac{z-a}{b}\right) H_{i}\left(\frac{z-a}{b}\right) H_{k}(z) d z, \quad \forall k \geq 0,
$$

which, with the simple change of variable $x=(z-a) / b$, becomes

$$
c_{k}=\sum_{i=0}^{2 m} \gamma_{i}(\boldsymbol{\nu}) E_{\phi}\left[H_{i}(x) H_{k}(a+b x)\right], \quad \forall k \geq 0,
$$

where $E_{\phi}[\cdot]$ is an expectation with respect to the standard normal density. The following proposition gives a general formula for these expectations:

Proposition 5 Let $x \sim N(0,1)$ with density $\phi(x)$. Then:

$$
E_{\phi}\left[H_{i}(x) H_{k}(a+b x)\right]=\sqrt{\frac{k !}{i !}} \sum_{j=0}^{\left\lfloor\frac{k-i}{2}\right\rfloor} \frac{H_{k-i-2 j}(a)}{j ! \sqrt{(k-i-2 j) !} 2^{j}} b^{i+2 j}
$$


for $i \leq k$ and zero otherwise, where $H_{i}(\cdot)$ is the $i$-th order standardised Hermite polynomial in (2) and $\lfloor\cdot\rfloor$ rounds its argument to the nearest integer toward zero.

In consequence, the coefficients of $z$ defined in (16) will be

$$
c_{k}=\sum_{i=0}^{\min (k, 2 m)} \sum_{j=0}^{\left\lfloor\frac{k-i}{2}\right\rfloor} \frac{\gamma_{i}(\boldsymbol{\nu})}{j ! 2^{j}} \sqrt{\frac{k !}{(i !)(k-i-2 j) !}} H_{k-i-2 j}(a) b^{i+2 j} .
$$

Finally, we can easily find the coefficients of the Gram-Charlier expansion of $z^{*}$ by substituting $a$ and $b$ by their respective values in (10). This expansion will generally be infinite except for one particular case. Specifically, if $\nu_{1}=\nu_{2}=0$ and $m>2$, then it can be shown that $c_{k}=0$ for $k>2 m$, since $a(\boldsymbol{\nu})=0$ and $b(\boldsymbol{\nu})=1$ in that case. Lim, Martin, and Martin (2005) have explored this restricted parametrisation with $m=4$ for option pricing purposes. In this paper, though, we will not impose any restrictions on the parameters of the SNP density.

\subsection{Comparison with other distributions}

Consider a truncated Gram-Charlier expansion of the form

$$
h\left(z^{+}\right)=\phi\left(z^{+}\right)\left[1+\sum_{i=3}^{n} c_{i} H_{i}\left(z^{+}\right)\right] .
$$

Notice that this density function has zero mean and unit variance by construction. In addition, if $n=2 m$, it involves exactly the same number of parameters as our standardised SNP variable $z^{*}$. However, as Jondeau and Rockinger (2001) point out, it is necessary to impose further restrictions on the parameters $c_{i}(i=3,4, \cdots, n)$ to ensure that the pdf in (18) is non-negative for all values of $z^{+} \in(-\infty, \infty)$. Unfortunately, they only determined those restrictions for $n=4$, because it becomes exceedingly difficult to find them for higher $n$. In contrast, we can leave the vector of parameters $\boldsymbol{\nu}$ free, except for a scale restriction, because positivity is always satisfied by a SNP density regardless of the expansion order.

Given that both $z^{*}$ and $z^{+}$have zero mean and unit variance, one may ask which of them leads to more general higher order moments. We will initially answer this question in terms of the third and fourth moments that these distributions can generate by plotting in Figure 1 the envelope of all the combinations of skewness and kurtosis for 
$m=2,3$ and 4 and $n=4 .^{2} \quad$ In addition, we have computed the regions of skewness and kurtosis generated by the VG distribution and the log of a GB variate. Finally, we also represent the skewness-kurtosis frontier that no density function can surpass (see e.g. Stuart and Ord, 1977). The advantage of the density in (18) is that the skewness and kurtosis coefficients can be directly obtained from $c_{3}$ and $c_{4}$. Nevertheless, the combinations of skewness and kurtosis that the variable $z^{+}$can generate are well within the combinations spanned by the SNP standardised variable $z^{*}$ with exactly the same number of free parameters, as we can see in Figure 1. For instance, while $z^{+}$could never be platykurtic, $z^{*}$ can indeed have kurtosis coefficients lower than 3. More importantly, the differences in minimum and maximum skewness are also substantial. Of course, by using the SNP instead of the Gram-Charlier expansion, we lose the direct interpretation of the parameters as skewness and kurtosis. However, this is also the case with many other non-Gaussian distributions, such as symmetric and asymmetric Student t distributions, and even the GB or VG ones. Finally, it is worth recalling that the SNP distribution guarantees positive densities regardless of $m$. In this sense, Figure 1 shows that we could achieve much more flexibility with just one or two additional parameters. As regards the other two models, we can observe that neither the GB nor the VG distributions can generate kurtosis below 3. It is also worth remarking that although the VG can generate infinite kurtosis, it cannot yield as high a skewness as the SNP for empirically relevant levels of kurtosis. In this sense, it can be shown that the frontier of the VG is obtained when this distribution converges to a Gamma. The GB also has limited flexibility, although it allows for higher skewness than Gram-Charlier expansions once positivity restrictions are imposed. In this case, it can be shown that the upper border of its frontier is obtained when the distribution of the log of a GB variate converges to an asymmetric double exponential, which becomes a single exponential at the two points of highest absolute skewness.

To get a clearer sense of the underlying differences between the distributions of $z^{+}$ and $z^{*}$, we can compare their Gram-Charlier expansions. ${ }^{3}$ Since both variables are

\footnotetext{
${ }^{2}$ We have used the procedure devised by Jondeau and Rockinger (2001) to obtain the frontier for a positive Gram-Charlier distribution with $n=4$, while we rely on (6) to represent the frontier of SNP densities with $m=2,3$ and 4 . To allow for $\nu_{0}=0$, we simulate 10 million parameters $\boldsymbol{\nu}$ in the unit sphere and compute the envelope of the values of skewness and kurtosis obtained from the simulated parameters.

${ }^{3}$ Note that (18) is already a proper Gram-Charlier expansion.
} 
standardised, both have $c_{0}=1$ and $c_{1}=c_{2}=0$. The third and fourth coefficients are functions of the skewness and kurtosis of the distributions, which we have already compared in the previous paragraph. Still, the main difference between $z^{*}$ and $z^{+}$is found in the higher order coefficients. In particular, whereas (18) imposes that $c_{k}=0$ for all $k>4$, such a restriction no longer holds for $z^{*}$. In other words, while the GramCharlier expansion of $z^{+}$is finite, the Gram-Charlier expansion of $z^{*}$ is generally infinite as we can see from (17).

\section{Option valuation}

\subsection{From the real to the risk neutral measure, and vice versa}

Consider a frictionless market with a risk free asset and a risky asset with price $S_{t}$ at time $t$. For any $T>t$, we can always express $S_{T}$ in terms of $S_{t}$ under the real measure $\mathbb{P}$ as:

$$
S_{T} \equiv S_{t} \exp \left[\left(\mu_{t}-\sigma_{t}^{2} / 2\right) \tau+\sigma_{t} \sqrt{\tau} z^{*}\right]
$$

where $\tau=T-t$ and $z^{*}$ is a random variable with zero mean and unit variance conditional on the information available at time t. In this context, $\mu_{t}$ and $\sigma_{t}$, which in general will be functions of the information known at $t$, represent the drift and volatility per unit of time of $S_{T}$. In what follows, we will assume that $z^{*}=a\left(\boldsymbol{\nu}_{t}\right)+b\left(\boldsymbol{\nu}_{t}\right) x^{\mathbb{P}}$, where $a\left(\boldsymbol{\nu}_{t}\right)$ and $b\left(\boldsymbol{\nu}_{t}\right)$ are defined in (10), and $x^{\mathbb{P}}$ is a SNP variate with shape parameters $\boldsymbol{\nu}_{t}$. With this notation, we can write the log-return as $y_{T}=\log \left(S_{T} / S_{t}\right)=\delta_{\mathbb{P} t}+\lambda_{\mathbb{P} t} x^{\mathbb{P}}$, where $\delta_{\mathbb{P} t}=\left(\mu_{t}-\sigma_{t}^{2} / 2\right) \tau+\sigma_{t} \sqrt{\tau} a\left(\boldsymbol{\nu}_{t}\right)$ and $\lambda_{\mathbb{P} t}=\sigma_{t} \sqrt{\tau} b\left(\boldsymbol{\nu}_{t}\right)$.

Our solution to the option pricing problem will be based on the use of a stochastic discount factor with an exponential affine form:

$$
M_{t, T}=\exp \left(\alpha_{t} y_{T}+\beta_{t} \tau\right)
$$

where again $\alpha_{t}$ and $\beta_{t}$ can be functions of the information known at time $t$. Such a specification corresponds to the Esscher transform used in insurance (see Esscher, 1932). In option pricing applications, this approach was pioneered by Gerber and Shiu (1994), and has also been followed by Buhlman, Delbaen, Embrechts, and Shyraev (1996, 1998), Gourieroux and Monfort (2006a,b) and Bertholon, Monfort, and Pegoraro (2003) among others. The following result provides the conditions for absence of arbitrage. For notational simplicity, we will drop the time subscripts for the remainder of the text. 
Proposition 6 Let $r$ be the risk-free rate and $I_{t}$ the information set at time $t$. If the conditional distribution of the log-return of the risky asset is a SNP of order $m$, then the stochastic discount factor (20) satisfies the arbitrage free conditions,

$$
E_{\mathbb{P}}\left[M_{t, T} \exp (r \tau) \mid I_{t}\right]=1, \quad E_{\mathbb{P}}\left[M_{t, T} \exp \left(y_{T}\right) \mid I_{t}\right]=1,
$$

if and only if

$$
\begin{aligned}
\sum_{k=0}^{2 m} \gamma_{k}(\boldsymbol{\nu}) \frac{\left(\alpha \lambda_{\mathbb{P}}\right)^{k}}{\sqrt{k !}} & =\exp \left[-\alpha \delta_{\mathbb{P}}-\frac{1}{2} \alpha^{2} \lambda_{\mathbb{P}}^{2}-\beta \tau-r \tau\right], \\
\sum_{k=0}^{2 m} \gamma_{k}(\boldsymbol{\nu}) \frac{(1+\alpha)^{k} \lambda_{\mathbb{P}}^{k}}{\sqrt{k !}} & =\exp \left[-(1+\alpha) \delta_{\mathbb{P}}-\frac{1}{2}(1+\alpha)^{2} \lambda_{\mathbb{P}}^{2}-\beta \tau\right] .
\end{aligned}
$$

From these two constraints, we can easily express $\beta$ as a function of $\alpha$. Hence, $\alpha$ can be obtained by solving a single non-linear equation, which is an implicit function of the remaining parameters of the model.

In this context, if $\mathbb{Q}$ denotes the risk neutral measure whose numeraire is the risk free asset, the real and risk-neutral measures can be easily related by means of the Radon-Nykodym derivative, which in this case is proportional to the discount factor

$$
\frac{d \mathbb{Q}}{d \mathbb{P}}=\frac{M_{t, T}}{E_{\mathbb{P}}\left(M_{t, T}\right)}
$$

Hence

$$
E_{\mathbb{Q}}\left(\digamma\left(S_{T}\right)\right)=E_{\mathbb{P}}\left[\frac{d \mathbb{Q}}{d \mathbb{P}} \digamma\left(S_{T}\right)\right],
$$

where $\digamma(\cdot)$ is an arbitrary function and $E_{\mathbb{P}}\left(M_{t, T}\right)=\exp (-r \tau)$, so that the discount factor correctly prices the risk-free asset. As a result, we can obtain the risk-neutral density from (24) as

$$
f^{\mathbb{Q}}\left(y_{T}\right)=\exp (r \tau) M_{t, T} f^{\mathbb{P}}\left(y_{T}\right) .
$$

On this basis, we can fully characterise the risk-neutral measure as follows:

Proposition 7 If the asset price $S_{T}$ is given by (19) under the real measure $\mathbb{P}$, where the distribution of its log return between $t$ and $T$ is a SNP of order $m$ with shape parameters $\boldsymbol{\nu}$, then it can be written under the risk neutral measure $\mathbb{Q}$ as

$$
S_{T}=S_{t} \exp \left[\left(\mu^{\mathbb{Q}}-\frac{\left(\sigma^{\mathbb{Q}}\right)^{2}}{2}\right) \tau+\sigma^{\mathbb{Q}} \sqrt{\tau} \kappa^{*}\right]
$$

where

$$
\mu^{\mathbb{Q}}=\mu+\frac{\sigma^{2}}{2}\left[\left(\frac{b(\boldsymbol{\nu})}{b(\boldsymbol{\theta})}\right)^{2}-1\right]+\frac{\sigma}{\sqrt{\tau}}\left[a(\boldsymbol{\nu})-a(\boldsymbol{\theta}) \frac{b(\boldsymbol{\nu})}{b(\boldsymbol{\theta})}\right]+\alpha \sigma^{2} b^{2}(\boldsymbol{\nu})
$$




$$
\sigma^{\mathbb{Q}}=\sigma b(\boldsymbol{\nu}) / b(\boldsymbol{\theta}),
$$

and $\kappa^{*}$ is a standardised SNP variable of order $m$ with shape parameters $\boldsymbol{\theta}=\left(\theta_{0}, \theta_{1}, \cdots, \theta_{m}\right)^{\prime}$, such that

$$
\theta_{i}=\sum_{k=i}^{m} \frac{\nu_{k}}{(k-i) !} \sqrt{\frac{k !}{i !}}\left(\alpha \lambda_{\mathbb{P}}\right)^{k-i}
$$

Therefore, in a SNP context the change of measure affects not only the mean and the variance of the log price, but also the higher moments, as can be seen from the differences between $\boldsymbol{\theta}$ and $\boldsymbol{\nu}$. For the case of $m=2$, for instance, we can show that the relation between $\boldsymbol{\theta}$ and $\boldsymbol{\nu}$ is $\theta_{0}=\nu_{0}+\nu_{1} \alpha \lambda_{\mathbb{P}}+\nu_{2} \alpha^{2} \lambda_{\mathbb{P}}^{2} / \sqrt{2}, \theta_{1}=\nu_{1}+\nu_{2} \sqrt{2} \alpha \lambda_{\mathbb{P}}$ and $\theta_{2}=\nu_{2}$. However, note that the SNP distribution is shared by the real and riskneutral measures. ${ }^{4} \quad$ Also, it is important to emphasise that this change of measure is always feasible because there are no restrictions on the shape parameters of the SNP distribution.

Obviously, our framework also allows us to value derivative assets by focusing on the risk-neutral measure directly without any reference to its relationship with the real measure, as in Jondeau and Rockinger (2001) or Jurczenko, Maillet, and Negrea (2002a,b). To follow this second approach, we just have to regard $\boldsymbol{\theta}, \mu^{\mathbb{Q}}$ and $\sigma^{\mathbb{Q}}$ as the structural parameters. The following proposition gives the expression that the risk-neutral drift must have to satisfy the martingale restriction (see Longstaff, 1995):

Proposition 8 If asset price $S_{T}$ is given by (26) under the risk-neutral measure $\mathbb{Q}$, where the distribution of its log return between $t$ and $T$ is a SNP of order $m$ with shape parameters $\boldsymbol{\theta}$, then the drift $\mu^{\mathbb{Q}}$ will satisfy the martingale restriction if and only if:

$$
\mu^{\mathbb{Q}}=r-(1 / \tau)\left[\sigma^{\mathbb{Q}} \sqrt{\tau} a(\boldsymbol{\theta})+(1 / 2)\left(\sigma^{\mathbb{Q}}\right)^{2} \tau\left(b^{2}(\boldsymbol{\theta})-1\right)+\log \Lambda\left(\boldsymbol{\theta}, \lambda_{\mathbb{Q}}\right)\right],
$$

where $\lambda_{\mathbb{Q}}=\sigma^{\mathbb{Q}} \sqrt{\tau} b(\boldsymbol{\theta})$ and $\Lambda(\cdot, \cdot)$ is defined in (8).

Not surprisingly, we show in appendix C that (27) and (30) coincide, which confirms that both strategies are indeed equivalent. This equivalence result has important computational advantages in empirical applications such as ours that only use option price data, because it allows one to estimate the option values from the risk neutral parameters without having to solve the nonlinear equations (22) and (23) within the optimisation

\footnotetext{
${ }^{4}$ Our results can be extended to more complicated specifications of the stochastic discount factor. For instance, an exponential quadratic form would also yield a SNP distribution of the same order under the risk-neutral distribution (the details are available upon request). In those cases, though, we would need to consider a larger number of assets in order to identify the parameters of the pricing kernel.
} 
algorithm. At the same time, if we had data on the underlying we could obtain the implied real-measure parameters. In particular, for a given drift $\mu$, risk-free rate $r$ and risk neutral parameters $\sigma^{\mathbb{Q}}$ and $\boldsymbol{\theta}$, we can recover the parameters of the real measure $\sigma$ and $\boldsymbol{\nu}$, together with the coefficient of relative risk aversion $\alpha$, from the following system of equations

$$
\begin{gathered}
\left(\mu-\sigma^{2} / 2\right) \tau+\sigma \sqrt{\tau} a(\boldsymbol{\nu})=\delta_{\mathbb{Q}}-\alpha \lambda_{\mathbb{Q}}^{2}, \\
\sigma \sqrt{\tau} b(\boldsymbol{\nu})=\lambda_{\mathbb{Q}}, \\
\nu_{i}=\sum_{k=i}^{m} \frac{\theta_{i}}{(k-i) !} \sqrt{\frac{k !}{i !}}(-1)^{k-i}\left(\lambda_{\mathbb{Q}} \alpha\right)^{k-i},
\end{gathered}
$$

where $\delta_{\mathbb{Q}}=\left(\mu^{\mathbb{Q}}-\sigma^{\mathbb{Q} 2} / 2\right) \tau+\sigma^{\mathbb{Q}} \sqrt{\tau} a(\boldsymbol{\theta})$. Finally, the discount factor $\beta$ can be obtained from either $(22)$ or $(23)$.

\subsection{Option pricing}

Let $C_{t}$ be the value at time $t$ of a European call option with strike price $K$ and expiration at time $T$, and let $S_{t}$ denote the underlying asset value. We can express $C_{t}$ as

$$
C_{t}=\exp (-r \tau) E_{\mathbb{Q}}\left[\left(S_{T}-K\right)^{+}\right]
$$

where $(\cdot)^{+}=\max (\cdot, 0)$. It is important to emphasise again the conditional nature of (32), which implies that all the parameters of the model can potentially depend on the information available at time t. If define the region $A=\left\{S_{T}>K\right\}$ we can rewrite (32) as

$$
C_{t}=\exp (-r \tau) E_{\mathbb{Q}}\left[S_{T} \mathbf{1}(A)\right]-K \exp (-r \tau) E_{\mathbb{Q}}[\mathbf{1}(A)]
$$

Following Geman, Karouri, and Rochet (1995), we can further simplify the calculations by changing the numeraire to the ratio of the risky asset prices $S_{T} / S_{t}$, which gives an alternative risk-neutral measure $\mathbb{Q}_{1}$. Then, if we use the Radon-Nikodym derivative:

$$
\frac{d \mathbb{Q}}{d \mathbb{Q}_{1}}=\frac{B_{T}}{B_{t}} \frac{S_{t}}{S_{T}}=\exp (r \tau) \frac{S_{t}}{S_{T}}
$$

we can easily express any expectation under $\mathbb{Q}$ in terms of $\mathbb{Q}_{1}$. Specifically, we will have that

$$
E_{\mathbb{Q}}\left[S_{T} \mathbf{1}(A)\right]=E_{\mathbb{Q}_{1}}\left[\frac{d \mathbb{Q}}{d \mathbb{Q}_{1}} S_{T} \mathbf{1}(A)\right]=S_{t} \exp (r \tau) E_{\mathbb{Q}_{1}}[\mathbf{1}(A)]
$$


which, once introduced in (33), gives us the general formula

$$
\begin{aligned}
C_{t} & =S_{t} E_{\mathbb{Q}_{1}}[\mathbf{1}(A)]-K \exp (-r \tau) E_{\mathbb{Q}}[\mathbf{1}(A)] \\
& =S_{t} \operatorname{Pr}_{\mathbb{Q}_{1}}\left[S_{T}>K\right]-K \exp (-r \tau) \operatorname{Pr}_{\mathbb{Q}}\left[S_{T}>K\right]
\end{aligned}
$$

The analytical tractability of the SNP distribution allows us to obtain closed form expressions for the probabilities in (35):

Proposition 9 The price at time $t$ of a European call option with strike $K$ written on the stock $S_{T}$ defined by (26) under the risk-neutral measure can be expressed as:

$$
C_{t}^{S N P}=S_{t} \operatorname{Pr}_{\mathbb{Q}_{1}}[x>d]-K \exp (-r \tau) \operatorname{Pr}_{\mathbb{Q}}[x>d]
$$

where

$$
\begin{gathered}
\operatorname{Pr}_{\mathbb{Q}}[x>d]=\Phi(-d)+\phi(d) \sum_{k=1}^{2 m} \frac{\gamma_{k}(\boldsymbol{\theta})}{\sqrt{k}} H_{k-1}(d), \\
\operatorname{Pr}_{\mathbb{Q}_{1}}[x>d]=\exp \left(-r \tau+\delta_{\mathbb{Q}}\right) \sum_{k=0}^{2 m} \gamma_{k}(\boldsymbol{\theta}) I_{k}^{*}, \\
I_{k}^{*}=\frac{1}{\sqrt{k}} \exp \left(\lambda_{\mathbb{Q}} d\right) H_{k-1}(d) \phi(d)+\frac{\lambda_{\mathbb{Q}}}{\sqrt{k}} I_{k-1}^{*} ; \quad I_{0}^{*}=\exp \left(\lambda_{\mathbb{Q}}^{2} / 2\right) \Phi\left(\lambda_{\mathbb{Q}}-d\right), \\
\delta_{\mathbb{Q}}=\left(\mu^{\mathbb{Q}}-\frac{\sigma^{\mathbb{Q} 2}}{2}\right) \tau+a(\boldsymbol{\theta}) \sigma^{\mathbb{Q}} \tau, \\
d=\frac{\log \left(K / S_{t}\right)-\delta_{\mathbb{Q}}}{\lambda_{\mathbb{Q}}} ; \lambda_{\mathbb{Q}}=b(\boldsymbol{\theta}) \sigma^{\mathbb{Q}} \sqrt{\tau}
\end{gathered}
$$

and $\Phi(\cdot)$ is the cumulative distribution function of the standard normal density.

As expected, (36) reduces to the Black and Scholes (1973) formula when $\theta_{0}=1$ and $\theta_{k}=0 \forall k \geq 1$. Importantly, if we treat the coefficients $\gamma_{k}$ of the Gram-Charlier expansion (4) as shape parameters themselves, instead of functions of either $\boldsymbol{\nu}$ or $\boldsymbol{\theta}$, we can show that (36) is also valid when the distribution of the underlying asset return is a finite Gram-Charlier expansion. As a consequence, we can use Proposition 9 to obtain closed form option prices when returns follow a high frequency process with iid SNP innovations, since Proposition 4 shows that their distribution at low frequencies is a Gram-Charlier expansion.

In Figure 2 we compare the range of call prices that the SNP density can produce with the corresponding ranges obtained for the Gram-Charlier expansion with positivity restrictions and the GB model. Not surprisingly, the higher flexibility of the SNP in 
modelling skewness and kurtosis that we saw in Figure 1 results in a wider range of call prices. The only exception is the VG model, which can reach the arbitrage bounds, but only under the limiting case in which the underlying distribution converges to a Bernoulli whose skewness tends to $+/$ - infinity. Importantly, a larger value of $m$ also leads to an SNP with even broader range. Nevertheless, there is a close relationship between the different pricing models: the Gram-Charlier call price formula can be obtained as a fourth-order Taylor expansion of (36), while Black-Scholes corresponds to a second-order one (see appendix A for further details).

\section{Semiparametric properties of the SNP option pric- ing model}

\subsection{Estimation with a misspecified model}

Fenton and Gallant (1996) and Gallant and Tauchen (1999) used the Marron and Wand (1992) test suite to analyse the semiparametric properties of SNP distributions in density estimation and in the implementation of the Efficient Method of Moments, respectively. However, their semiparametric properties in option pricing applications have not been studied. In this section, we will assess the performance of our option pricing model when the true distribution is not SNP.

Specifically, we will assume that the true distribution of the underlying asset return is one of the first nine non-Gaussian distributions proposed by Marron and Wand (1992). For each of them, we generate 1000 call option prices from the true model, with a range of moneyness uniformly distributed between \pm 3 times the standard deviation of the underlying asset return. Finally, we estimate the parameters of the following misspecified models by minimising the root mean square pricing errors (RMSE's): Black-Scholes, Gram-Charlier with two shape parameters, SNP with $m=2$, and SNP of order $m^{*}$ such that the RMSE divided by the mean option price is less than 10 basis points.

The results are displayed in Figures 3a to 3c. The left panels show the shape of the true density, whereas the right panels display the true implied volatilities together with the ones estimated with the misspecified models. Since none of these models is Gaussian, Black-Scholes performs poorly in most cases. The models with two shape parameters perform reasonably well in some examples, such as the skewed and kurtotic unimodal cases. However, in some other examples, such as the strongly skewed, the Gram-Charlier 
parameter estimates cannot guarantee the positivity of the density. The consequence is that Gram-Charlier implied volatilities suddenly jump to zero for some ranges of the moneyness. In contrast, our pricing model does not suffer from this restriction. Of course, if we let $m \rightarrow \infty$ then we will be able to exactly reproduce all the volatility smiles. However, we are able to show that even for finite $m$, the SNP already performs very well. In this sense, we can check that we obtain substantial improvements in fit in all cases as we increase the order of the SNP. For example, the rather complicated shape of the Claw implied volatility is already well captured with $m^{*}=13$ (see Figure 3c).

\subsection{Temporal aggregation}

From Proposition 4, we know that the distribution of aggregated SNP returns is not a SNP of the same order, not even when they are iid. In this subsection, we assess the ability of our model applied to low frequency data to fit option prices that have been generated with a high frequency process with SNP log-returns. To do so, we model the weekly process of log-returns with a non-iid SNP distribution of order $m=2$, whose volatility follows a persistent binary Markov chain with parameters calibrated using S\&P 500 weekly return data from 1950 to $2006 .^{5}$ Then, we have generated from this process 1000 option prices maturing in one and three months with the same range of moneyness as in the previous subsection. We fit SNP's of increasing order to these prices until the RMSE divided by the mean option price is less than 10 basis points.

As shown in Figure 4, a SNP with $m=4$ is enough to yield a RMSE below our target for both maturities. This is somewhat surprising if we take into account that, for a given volatility path, the distribution of the one and three month log-returns are Gram-Charlier expansions of order 16 and 48, respectively (see Proposition 4). Thus, we believe that the time incoherence problem should not be an issue of major concern in our context. We can also notice in Figure 4 the flattening of the smile at the longer horizon, a feature that is consistent with the empirical evidence (see e.g. Das and Sundaram, 1999).

\footnotetext{
${ }^{5}$ Although for the purposes of our exercise we could have considered a continuous distribution for volatility, we have chosen a Markov chain only because we can obtain closed form option prices. Specifically, for a given a volatility path the distribution of the log return between the initial and final dates is just a Gram-Charlier expansion, for which Proposition 9 applies. Hence, we can express the actual call price as a weighted sum of the option prices in each possible path, with weights that correspond to their probability of occurring.
} 


\section{Empirical performance of SNP option pricing}

In this section, we apply the SNP option valuation formula (36) in Proposition 9 to S\&P 500 index options using the same database as Dumas, Fleming, and Whaley (1998). Option prices were collected every Wednesday between 2:45 p.m. and 3:45 p.m. from June 1988 to December 1993, which makes a total number of 292 days. Options are European-style and expire on the third Friday of each contract month. We will focus on call options, and use the bid-ask mid price for estimation purposes. The riskless interest rate will be proxied by the T-bill rate implied by the average of the bid and ask discounts reported in the Wall Street Journal. To account for the presence of dividends, the implied forward price is computed as the current stock price $S_{t}$ minus the present value of dividends $\bar{D}_{t}$ times the interest accrued until maturity, i.e. $F_{t, T}=\left(S_{t}-\bar{D}_{t}\right) \exp (r \tau)$ (see Dumas, Fleming, and Whaley, 1998, for further details).

We will compare the performance of the SNP option valuation framework with the following competing models: the standard Black and Scholes (1973) model, the GramCharlier expansion with positivity restrictions, the GB and VG models, and finally a variant of the Black-Scholes model where the volatility is assumed to be a quadratic function of moneyness. We will call this methodology Practitioners' Black-Scholes, a name inspired by its wide use in the financial industry. In order to guarantee positivity, we will consider the parametrisation

$$
\sigma(x)=\rho_{0}+\rho_{1}\left(x-\rho_{2}\right)^{2}
$$

where $\rho_{0}>0, \rho_{1} \geq 0$ and $x=F_{t, T} / K$. Finally, note that since we are using implied forward prices, an adjustment in the spirit of Black (1976) is needed in all cases.

We consider separate estimations for short and long maturities. Both are carried out by minimising the sum of squared pricing errors. To select the short maturity group, we begin by considering call options that mature in 45 days for the first day in the sample. We track those options every week until two weeks before they expire. Then, we move to the next group of options that are 45 days away from expiration and start the tracking process again. At the end, we have data on 3,462 call option prices with median time to expiration of 24 days, and a number of options per day that ranges from 4 to 25 , with a median of 11. In the long maturity group we follow an analogous selection process. In particular, we have selected 4,306 call option prices with a median time to maturity of 
150 days. The number of prices per day also ranges between 4 and 25, but the median is now 15 .

Tables 1a to 1d report the RMSE's of the six competing models when we allow all the parameters of the conditional distribution of returns to vary each Wednesday, which is consistent with the conditional nature of our pricing framework. We also provide information on the degree of fit achieved for different degrees of moneyness using the six categories proposed by Bakshi, Cao, and Chen (1997), together with the number of options in each category. Tables 1a and 1c report in-sample RMSE's based on the first four years of data. In contrast, Tables $1 \mathrm{~b}$ and $1 \mathrm{~d}$ report out-of-sample results based on pricing errors for each Wednesday in the last year of the sample using the parameters estimated on the previous Wednesday. In the short maturity group, Practitioners's Black-Scholes and the SNP are the two best performing models in-sample, followed by the VG and GB models. However, if we look at the out-of-sample results, we can observe that Practitioner's Black-Scholes shows a strong parameter instability, whereas the other three models are much more stable. In the long maturity group, again the SNP, GB and VG models yield the lowest RMSE's, although VG yields a slightly better fit in this case. Nevertheless, the differences between these three models are very small, whereas the RMSE's of the Black-Scholes, Gram-Charlier with positivity restrictions and Practitioners' Black-Scholes models are clearly higher.

In Figures 5a and 5b we have plotted the skewness and kurtosis values implied by the SNP, Gram-Charlier with positivity restrictions, GB and VG models for each day in the in-sample period. Several important patterns arise from these figures. First, there is high dispersion in the estimated higher order moments, although skewness is usually negative and kurtosis is typically higher than 3. Second, skewness and kurtosis tend to be lower when the time to expiration is longer. Furthermore, skewness and kurtosis in Gram-Charlier densities with positivity restrictions are usually on the frontier of values compatible with these densities. This is also observed with the VG and specially with the GB model. In particular, market prices often suggest a more (negative) skewness than these models are able to account for. However, some SNP estimates are also located on the frontier, especially in the short maturity group. Although we could easily enlarge the SNP frontier by simply increasing the order $m$ (see Figure 1), it is interesting to analyse in more detail the possible sources of the high sampling variability. 
To do so, we have carried out the following bootstrap exercise. First, we group the SNP pricing errors obtained for short maturities in the six moneyness categories already considered. Then, we simulate prices for a specific but broadly representative day (November 13, 1991), by adding random pricing errors to the 19 prices of that day estimated with the SNP model. In this sense, we sample the errors that we add to each price from the same moneyness category to which that price belongs. In this way, we take into account possible distributional differences between pricing errors for, say, deep in the money and out of the money options. Finally, we re-estimate the SNP model on the simulated data. We plot the implied skewness and kurtosis for 1,000 such simulations in Figure 5c. As we can observe, the estimates are again highly disperse, and basically cover the whole region of negative skewness. Nevertheless, the true option prices have constant parameters by construction, which approximately correspond to skewness of -1.5 and kurtosis of 7.7 (see Figure 5c).

Therefore, it may well be the case that even if the true parameters are constant, the high variation in skewness and kurtosis that we observe in Figures 5a and 5b simply results from the relatively low number of prices with which we are estimating the daily models. For that reason, we also study the performance of all the different models under the assumption that the conditional distribution of standardised log-returns (or $\rho_{1}$ and $\rho_{2}$ in (38)) is time invariant, while volatility (or the intercept $\rho_{0}$ in Practitioner's BlackScholes) is allowed to change over time as before. Again, we carry out an in-sample and an out-of-sample analysis, which show that the SNP, GB and VG models perform more or less on the same level, while the remaining models yield less satisfactory results (see Tables 2 a to 2d.). We can also note that, by increasing the order of the SNP we can improve its performance without deteriorating its out of sample stability.

If we compare the SNP pricing errors in Tables $2 \mathrm{~b}$ and $2 \mathrm{~d}$ with those of Tables $1 \mathrm{~b}$ and $1 \mathrm{~d}$, we can observe that the assumption of constant shape parameters does indeed yield better out-of-sample results. Importantly, the SNP with fixed parameters generally performs better out-of-sample than the remaining models with time varying parameters. In terms of skewness and kurtosis, Figure 5d shows that SNP estimations are no longer at the frontier. In contrast, Gram-Charlier and GB estimates are very close or exactly on their respective frontiers.

As a sanity check, Figure 6 confirms that the main differences between Black-Scholes 
and the remaining non-Gaussian models lie in the tails of the distributions, and not so much in the temporal evolution of the volatilities.

Another interesting issue is whether the main reason for the rejection of the BlackScholes model is skewness or excess kurtosis. To find out, we have re-estimated our SNP model for $m=2$ with fixed parameters imposing zero skewness first, and then kurtosis equal to 3. Interestingly, it turns out that when we force the skewness to be zero we obtain the Black-Scholes special case. In contrast, if we fix the kurtosis to 3, we obtain substantial negative skewness for both the short and long maturity groups. Hence, it seems that negative skewness plays a more fundamental role in determining option prices than excess kurtosis.

Finally, we compare the estimated conditional risk-neutral densities in Figures 7a to $7 \mathrm{~d}$ for the same day as in the bootstrap exercise, having obtained the density implied by the Practitioner's Black-Scholes model from the second derivative of the call price with respect to the strike (see Breeden and Litzenberger, 1978). All the models except BlackScholes imply negatively skewed and peaked densities, but they are reasonably similar at the centre, except for the much higher peaks in the VG densities. In fact, it turns out that this density has a pole near zero for the long maturity group. However, zooms of the left tails show that the Practitioner's Black-Scholes model attaches unreasonably high probabilities to extreme negative events. This result is consistent with the fact that the Practitioner's Black-Scholes method gives relatively good results in-sample but unrealistic implications for out-of-the-money calls. In Figure 8 we compare the smiles that each model can generate with the bid, ask and mid-price quotes for our chosen representative day. Practitioner's Black-Scholes tries to fit a quadratic curve to the smile, at the cost of not providing very reliable results at the extremes (see in particular the out-of-the money area). This picture also shows that the rather limited amount of skewness allowed by "positive" Gram-Charlier densities prevents them from reproducing the empirical smile as we get deeper in the money. However, lack of liquidity is stronger in deep in-the-money options, so the real importance of this result must be taken with some caution. 


\section{$6 \quad$ Extensions}

The SNP density of order $m$ is constructed by multiplying the Gaussian density by a squared polynomial of order $m$. The fact that the polynomial of the expansion is a perfect square is a sufficient but not necessary condition for positivity of the final density. Hence, we can create a generalised SNP (GSNP) density by multiplying the Gaussian density with an otherwise unrestricted positive polynomial $P_{2 m}(x)$ of order $2 m$. This distribution will include as particular cases both the SNP and the Gram-Charlier density with positivity restrictions.

The positivity of $P_{2 m}(x)$ can be ensured by forcing its roots to be either real and double, or complex conjugates. In contrast, in the SNP case the complex roots must always be double. Meddahi (2001) shows that a necessary and sufficient condition for $P_{2 m}(x)$ to be positive is that it can be written as the sum of two squared polynomials of order $m$.

Interestingly, we can interpret the GSNP density as a mixture of two SNP densities with the same location and scale:

Proposition 10 The GSNP density can be written as

$$
f_{G S N P}\left(x ; \boldsymbol{\nu}_{1}, \boldsymbol{\nu}_{2}\right)=p\left(\boldsymbol{\nu}_{1}, \boldsymbol{\nu}_{2}\right) f\left(x ; \boldsymbol{\nu}_{1}\right)+\left[1-p\left(\boldsymbol{\nu}_{1}, \boldsymbol{\nu}_{2}\right)\right] f\left(x ; \boldsymbol{\nu}_{2}\right)
$$

where $\boldsymbol{\nu}_{1}$ and $\boldsymbol{\nu}_{2}$ are vectors of dimension $m$ and $m-1$, respectively, $f\left(x ; \boldsymbol{\nu}_{1}\right)$ is defined in (1), and

$$
p\left(\boldsymbol{\nu}_{1}, \boldsymbol{\nu}_{2}\right)=\frac{\boldsymbol{\nu}_{1}^{\prime} \boldsymbol{\nu}_{1}}{\boldsymbol{\nu}_{1}^{\prime} \boldsymbol{\nu}_{1}+\boldsymbol{\nu}_{2}^{\prime} \boldsymbol{\nu}_{2}}
$$

This interpretation can be exploited to extend the results of the paper to this generalised class of distributions. Nevertheless, despite the increased generality of the GSNP, we have found that it does not seem to provide a higher flexibility in terms of skewness, kurtosis or range of option prices than a standard SNP density of the same order. In any case, we leave a more thorough study of the characteristics of the GSNP density for future research.

\section{Conclusions}

In this paper we propose the use as a parametric model of the SNP distribution introduced by Gallant and Nychka (1987) for nonparametric estimation purposes. The SNP distribution shares the analytical tractability of truncated Gram-Charlier densities, but, 
unlike them, it has a density function that is always positive. From the statistical point of view, we give expressions for the moments and show that the distribution of linear combinations of SNP variables can be expressed as a finite Gram-Charlier expansion. We also construct a standardised SNP variable and compare it with the standardised Gram-Charlier random variable with positivity restrictions on its density, as well as the Generalised Beta and Variance Gamma distributions. In this sense, we show that the SNP distribution provides more flexibility in terms of both skewness and kurtosis. And although the SNP cannot yield unlimited kurtosis as the VG, this problem does not seem to be relevant in practice.

Next, we focus our attention on option pricing. In this respect, we show that if the log of the underlying asset price has a conditional SNP distribution under the real measure, and the stochastic discount factor is exponentially affine, which is equivalent to the Esscher transform, the log of the underlying asset price will also have a conditional SNP distribution of the same order under the risk-neutral measure. On this basis, we obtain closed form expressions for European option prices. Alternatively, we can obtain equivalent option prices by directly assuming that the log of the underlying asset price follows a SNP distribution under the risk-neutral measure, although in this case we must first determine the risk-neutral drift which guarantees that the martingale restriction is satisfied. We also relate our pricing formulas to the ones of Black and Scholes (1973), Corrado and Su (1996, 1997), and Jurczenko, Maillet, and Negrea (2002a). In this respect, we show that their formulas can be obtained as second and fourth order Taylor expansions of our formulas, respectively.

We also assess the semiparametric properties of our model when it is misspecified. Specifically, we generate option prices from the non-Gaussian densities proposed by Marron and Wand (1992), and show that our SNP option pricing formula can approximate arbitrarily well the prices of options whose true densities are not SNP, however complicated they may be.

Furthermore, although our model is not closed under temporal aggregation, we show that our pricing formulas can be applied to obtain exact option prices in the context of a high frequency SNP model. In addition, we also show that a low order SNP can approximate very well the behaviour of low frequency option prices generated by a stochastic volatility high frequency process with SNP innovations. 
Finally, we carry out an empirical application to the S\&P 500 options data of Dumas, Fleming, and Whaley (1998) in which we evaluate the performance of our pricing formulas using the Black and Scholes (1973) model as a benchmark. We also compare our model with the so-called Practitioner's Black-Scholes procedure, which fits a quadratic polynomial to the volatility smile, the Gram-Charlier density with positivity restrictions, as well as the GB and VG models. We find that the SNP, together with the GB and the VG, are the best performing models in general terms, both in and out of sample. Interestingly, we also find a high dispersion in the daily estimates of skewness and kurtosis. However, we show with a bootstrap exercise that this effect is probably due to sampling variability. In this sense, we find that the pricing performance of our model improves out-of-sample if we keep the shape parameters constant over time. It is also worth mentioning that although the empirical rejection of the Black-Scholes model is due to the presence of both negative skewness and excess kurtosis, skewness seems to be relatively more important than excess kurtosis.

As an extension, we provide a generalised version of the SNP distribution that nests all positive Gram-Charlier expansions as particular cases, including the fourth order one considered by Jondeau and Rockinger (2001). Importantly, we show that such generalised SNP distributions can be generated from a mixture of two SNP variables with the same location and scale, which allows us to extend our previous results to this new class of densities.

A fruitful avenue for future research would be to exploit the relationship between real and risk-neutral measures in the estimation of our option pricing model by combining data on the underlying asset price, which is informative about the real measure, with option price data, which contains information about the risk-neutral measure (see Jackwerth, 2000). It would also be interesting to explore possible time varying specifications for the parameters of the model, such as GARCH parametrisations for the volatility (see Heston and Nandi, 2000), and analogous extensions for the remaining shape parameters, as in Hansen (1994) or Jondeau and Rockinger (2005). Similarly, it would also be worth exploring the flexibility of the SNP and generalised SNP distributions for risk-management purposes. 


\section{References}

Bakshi, G., C. Cao, and Z. Chen (1997). Empirical performance of alternative option pricing models. The Journal of Finance 52, 2003-2049.

Bertholon, H., A. Monfort, and F. Pegoraro (2003). Pricing and inference with mixtures of conditionally normal processes. Mimeo CREST.

Black, F. (1976). The pricing of commodity contracts. Journal of Financial Economics 3, 167-179.

Black, F. and M. Scholes (1973). The pricing of options and corporate liabilities. Journal of Political Economy, 637-655.

Bollerslev, T. and J. Wooldridge (1992). Quasi maximum likelihood estimation and inference in dynamic models with time-varying covariances. Econometric Reviews 11, $143-172$.

Bontemps, C. and N. Meddahi (2005). Testing normality: a GMM approach. Journal of Econometrics 124, 149-186.

Bookstaber, R. and J. B. McDonald (1987). A general distribution for describing security price returns. Journal of Business 60, 401-424.

Breeden, D. T. and R. H. Litzenberger (1978). Prices of state-contingent claims implicit in option prices. The Journal of Business 51, 621-651.

Buhlman, H., F. Delbaen, P. Embrechts, and A. Shyraev (1996). No arbitrage, change of measure and conditional Esscher transforms in a semi-martingale model of stock processes. CWI Quarterly 9, 291-317.

Buhlman, H., F. Delbaen, P. Embrechts, and A. Shyraev (1998). On Esscher transforms in discrete finance models. ASTIN Bulletin 28, 171-186.

Capelle-Blanchard, G., E. Jurczenko, and B. Maillet (2001). The approximate option pricing model: performances and dynamic properties. Journal of Multinational Financial Management 11, 427-444.

Corrado, C. and T. Su (1996). Skewness and kurtosis in S\&P 500 index returns implied by option prices. Journal of Financial Research 19, 175-192.

Corrado, C. and T. Su (1997). Implied volatility skews and stock return skewness and kurtosis implied by stock option prices. European Journal of Finance 3, 73-85.

Das, S. R. and R. K. Sundaram (1999). Of smiles and smirks: a term structure perspective. Journal of Financial and Quantitative Analysis 34, 211-239.

Davidson, R. and J. G. MacKinnon (1993). Estimation and Inference in Econometrics. 
Oxford, U.K.: Oxford University Press.

Dumas, B., J. Fleming, and E. Whaley (1998). Implied volatility functions: empirical tests. The Journal of Finance 53, 2059-2106.

Esscher, F. (1932). On the probability function in the collective theory of risk. Skandinavisk Aktuariedskrift 15, 165-195.

Fenton, V. and A. R. Gallant (1996). Qualitative and asymptotic performance of SNP density estimators. Journal of Econometrics 74, 77-118.

Gallant, A. and G. Tauchen (1999). The relative efficiency of method of moment estimators. Journal of Econometrics 92, 149-172.

Gallant, A. R. and D. W. Nychka (1987). Seminonparametric maximum likelihood estimation. Econometrica 55, 363-390.

Geman, H., N. E. Karouri, and J.-C. Rochet (1995). Changes of numeraire, changes of probability and option pricing. Journal of Applied Probability 32, 443-458.

Gerber, H. and E. Shiu (1994). Option pricing by Esscher transforms. Transactions of Society of Actuaries 46, 99-140.

Gourieroux, C. and A. Monfort (2006a). Econometric specification of stochastic discount factor models. Forthcoming Journal of Econometrics.

Gourieroux, C. and A. Monfort (2006b). Pricing with splines. Forthcoming Annales de Economie et de Statistique.

Hansen, B. E. (1994). Autoregressive conditional density estimation. International Economic Review 35, 705-730.

Heston, S. L. and S. Nandi (2000). A closed-form GARCH option valuation model. The Review of Financial Studies 13, 585-625.

Jackwerth, J. C. (2000). Recovering risk aversion from option prices and realized returns. Review of Financial Studies 13, 433-451.

Jarrow, R. and A. Rudd (1982). Approximate option valuation for arbitrary stochastic processes. Journal of Financial Economics 10, 347-369.

Jondeau, E. and M. Rockinger (2001). Gram-Charlier densities. Journal of Economic Dynamics and Control 25, 1457-1483.

Jondeau, E. and M. Rockinger (2005). Conditional asset allocation under non-normality: how costly is the mean-variance criterion? Mimeo HEC Lausanne.

Jurczenko, E., B. Maillet, and B. Negrea (2002a). Multi-moment option pricing models: a general comparison (part 1). Working paper, University of Paris I Panthéon-Sorbonne.

Jurczenko, E., B. Maillet, and B. Negrea (2002b). Skewness and kurtosis implied by 
option prices: a second comment. FMG discussion paper DP419.

Lim, G. C., G. M. Martin, and V. L. Martin (2005). Parametric pricing of higher order moments in S\&P500 options. Journal of Applied Econometrics 20, 377-404.

Liu, X., M. B. Shackleton, S. J. Taylor, and X. Xu (2006). Closed-form transformations from risk-neutral to real-world distributions. Journal of Banking and Finance (Forthcoming).

Longstaff, F. A. (1995). Option pricing and the martingale restriction. The Review of Financial Studies 8, 1091-1124.

Madan, D. B., P. P. Carr, and E. C. Chang (1998). The Variance Gamma process and option pricing. European Finance Review 2, 79-105.

Madan, D. B. and F. Milne (1991). Option pricing with V.G. martingale components. Mathematical Finance 1, 39-55.

Marron, J. S. and M. P. Wand (1992). Exact mean integrated squared error. The Annals of Statistics 20, 712-736.

Meddahi, N. (2001). An eigenfunction approach for volatility modeling. Université de Montréal Working Paper 29-2001.

Stuart, A. and K. Ord (1977). Kendall's Advanced Theory of Statistics (6th ed.), Volume 1. London: Griffin. 


\section{A Relationship with Gram-Charlier option pricing models}

We can express (36) in terms of the infinite Gram-Charlier expansion the SNP distribution as follows:

Proposition 11 The call price $C_{t}^{S N P}$ in (36) can be rewritten in terms of an infinite expansion $C_{t}^{S N P}=\xi_{0}+\xi_{3} s k+\xi_{4}(k u-3)+\zeta$, where

$$
\zeta=e^{-r \tau} \sum_{k=5}^{\infty} c_{k} \int_{\omega}^{\infty}\left(S_{T}\left(\kappa^{*}\right)-K\right) H_{k}\left(\kappa^{*}\right) \phi\left(\kappa^{*}\right) d \kappa^{*},
$$

$$
\begin{aligned}
& \xi_{0}=S_{t} e^{\left(\mu^{\mathbb{Q}}-r\right) \tau} \Phi\left(d_{1}^{*}\right)-K e^{-r \tau} \Phi\left(d_{1}^{*}-\sigma_{\tau}\right), \\
& \xi_{3}=(1 / 3 !) \sigma_{\tau} S_{t} e^{\left(\mu^{\mathbb{Q}}-r\right) \tau}\left[\sigma_{\tau}^{2} \Phi\left(d_{1}^{*}\right)+\left(2 \sigma_{\tau}-d_{1}^{*}\right) \phi\left(d_{1}^{*}\right)\right], \\
& \xi_{4}=(1 / 4 !) \sigma_{\tau} S_{t} e^{\left(\mu^{\mathbb{Q}}-r\right) \tau}\left[\sigma_{\tau}^{3} \Phi\left(d_{1}^{*}\right)+\left(3 \sigma_{\tau}^{2}-3 d_{1}^{*} \sigma_{\tau}+d_{1}^{* 2}-1\right) \phi\left(d_{1}^{*}\right)\right],
\end{aligned}
$$

$\omega=\sigma_{\tau}-d_{1}^{*}, \sigma_{\tau}=\sigma^{\mathbb{Q}} \sqrt{\tau}, d_{1}^{*}=\left[\log \left(S_{t} / K\right)+\left(\mu^{*}+\sigma^{2} / 2\right) \tau\right] / \sigma_{\tau}$ and $S_{T}\left(\kappa^{*}\right)$, defined in (26), is regarded as a function of the standardised random variable $\kappa^{*}$, while the coefficients $c_{k}$ are given in (17).

We can use Proposition 11 to relate our pricing model to the model of Corrado and $\mathrm{Su}(1996,1997)$, who consider a fourth order Gram-Charlier density $\left(c_{k}=0\right.$, for $k \geq 5$ in (13)), without imposing positivity restrictions. In this respect, it is important to mention that the original Corrado-Su formula, apart from containing a mistake in the definition of the Hermite polynomials, does not satisfy the martingale restriction (32). Both problems are dealt by Jurczenko, Maillet, and Negrea (2002b). The following result shows that the martingale restriction in Jurczenko, Maillet, and Negrea (2002b) can be regarded as a truncated version of our drift (30):

Lemma 2 The drift of the risk neutral price model can be written as $\mu^{\mathbb{Q}}=r-(1 / \tau) \log \left[1+(s k / 3 !) \sigma_{\tau}^{3}+((k u-3) / 4 !) \sigma_{\tau}^{4}+o\left(\sigma_{\tau}^{4}\right)\right]$.

On this basis, it is easy to show that the modified Corrado-Su formula is an approximated version of our call formula in which we only retain the first four elements of a Taylor expansion in $\sigma_{\tau}$ of the SNP call pricing formula:

Proposition 12 Consider the call price $C_{t}^{S N P}$ in Proposition 11. Then, if we neglect the term $\zeta, C_{t}^{S N P}$ can be written as $C_{t}^{S N P}=C_{t}^{* C S}+o\left(\sigma_{\tau}^{4}\right)$, where $C_{t}^{* C S}$ is the modified Corrado-Su formula (see Jurczenko et al., 2002b)

$$
C_{t}^{* C S}=C_{t}^{* B S}+s k Q_{3}^{*}+(k u-3) Q_{4}^{*},
$$




$$
\begin{gathered}
C_{t}^{* B S}=S_{t} \Phi\left(d^{*}\right)-K e^{-r \tau} \Phi\left(d^{*}-\sigma_{\tau}\right), \\
d^{*}=\left(1 / \sigma_{\tau}\right)\left[\log \left(S_{t} / K\right)+\left(r+\frac{\sigma^{2}}{2}\right) \tau\right]-\left(1 / \sigma_{\tau}\right) \log \left(1+\frac{s k}{3 !} \sigma_{\tau}^{3}+\frac{(k u-3)}{4 !} \sigma_{\tau}^{4}\right), \\
Q_{3}^{*}=(1 / 3 !) \sigma_{\tau} S_{t}\left(2 \sigma_{\tau}-d^{*}\right) \phi\left(d^{*}\right)\left(1+(1 / 3 !) s k \sigma_{\tau}^{3}+(1 / 4 !)(k u-3) \sigma_{\tau}^{4}\right)^{-1},
\end{gathered}
$$

and

$Q_{4}^{*}=(1 / 4 !) \sigma_{\tau} S_{t}\left(3 \sigma_{\tau}^{2}-3 d^{*} \sigma_{\tau}+d^{* 2}-1\right) \phi\left(d^{*}\right)\left(1+(1 / 3 !) s k \sigma_{\tau}^{3}+(1 / 4 !)(k u-3) \sigma_{\tau}^{4}\right)^{-1}$.

The main difference between the SNP model and the modified Corrado-Su formula results from the fact that Corrado and $\mathrm{Su}$ do not impose positivity restrictions on the density. In fact, a statistically correct version of the Corrado-Su model should impose the positivity restrictions of Jondeau and Rockinger (2001). Hence, our SNP assumption, which implicitly guarantees a non-negative density, leads to a slightly more complex formula for the same number of parameters (i.e., for $m=2$ ). However, as Proposition 12 shows, if we eliminate the higher order terms in the infinite expansion of Proposition 11, the same fundamental effects of skewness and kurtosis emerge. Furthermore, if we neglect the terms $\sigma_{\tau}^{k}$ for $k \geq 3$ in a Taylor expansion of (A1) we can relate the SNP and the Black-Scholes model with the following result:

Proposition 13 We can write $C_{t}^{S N P}$ as

$$
C_{t}^{S N P}=C_{t}^{B S}+\beta_{3} s k+\beta_{4}(k u-3)+o\left(\sigma_{\tau}^{2}\right),
$$

where $C_{t}^{B S}$ is the Black-Scholes formula, $d_{1}=\left[\log \left(S_{t} / K\right)+\left(r+(1 / 2) \sigma^{2}\right) \tau\right] /(\sigma \sqrt{\tau})$ and

$$
\beta_{3}=(1 / 3 !) S_{t} \sigma_{\tau}\left(\sigma_{\tau}-d_{1}\right) \phi\left(d_{1}\right)+(1 / 3 !) K \exp (-r \tau) \phi\left(d_{1}\right) \sigma_{\tau}^{2},
$$

and

$$
\beta_{4}=(1 / 4 !) S_{t} \sigma_{\tau}\left(d_{1}^{2}-3 d_{1} \sigma_{\tau}-1\right) \phi\left(d_{1}\right)
$$

An analogous result is provided in Jurczenko, Maillet, and Negrea (2002b) for the modified Corrado-Su formula, under the name of "Simplified Corrado-Su formula". However, we will not obtain exactly the formula since Jurczenko, Maillet, and Negrea (2002b) approximate $d^{*}$ by $d_{1}$, which implies that they are effectively discarding some terms in $\sigma_{\tau}^{2}$. We can also provide an approximate expression for the implied volatility in the SNP model:

Proposition 14 Let $C_{t}^{S N P}$ denote the market price on a European call option. Then the implied volatility $\Psi$ for a given moneyness and time to maturity can be written as

$$
\Psi \simeq \sigma \sqrt{\tau}+\widetilde{\beta}_{3} s k+\widetilde{\beta}_{4}(k u-3),
$$

where $\widetilde{\beta}_{3}=(1 / 3 !) \sigma_{\tau}\left(2 \sigma_{\tau}-d_{1}\right)+(1 / 3 !)\left(K / S_{t}\right) \exp (-r \tau) \sigma_{\tau}^{2}$, and $\tilde{\beta}_{4}=(1 / 4 !) \sigma_{\tau}\left(d_{1}^{2}-3 d_{1} \sigma_{\tau}-1\right)$. 
Table 1

(a) In-sample RMSE for the short maturity group with time-varying parameters.

\begin{tabular}{cccccccc}
\hline Moneyness & BS & Pr. BS & GC $^{+}$ & $\mathrm{SNP}(\mathrm{m}=2)$ & G. Beta & V. Gamma & $\mathrm{N}$ \\
\hline \hline$<0.94$ & 0.488 & 0.127 & 0.213 & 0.104 & 0.086 & 0.113 & 65 \\
$0.94-0.97$ & 0.542 & 0.137 & 0.201 & 0.110 & 0.106 & 0.141 & 287 \\
$0.97-1.00$ & 0.489 & 0.143 & 0.175 & 0.124 & 0.125 & 0.179 & 450 \\
$1.00-1.03$ & 0.291 & 0.176 & 0.144 & 0.140 & 0.213 & 0.173 & 439 \\
$1.03-1.06$ & 0.662 & 0.160 & 0.167 & 0.129 & 0.172 & 0.189 & 434 \\
$>1.06$ & 0.732 & 0.284 & 0.435 & 0.334 & 0.436 & 0.330 & 1,176 \\
Total & 0.611 & 0.218 & 0.309 & 0.236 & 0.306 & 0.249 & 2,851 \\
\hline
\end{tabular}

(b) Out-of-sample RMSE for the short maturity group with time-varying parameters.

\begin{tabular}{cccccccc}
\hline Moneyness & $\mathrm{BS}$ & Pr. BS & $\mathrm{GC}^{+}$ & $\mathrm{SNP}(\mathrm{m}=2)$ & G. Beta & V. Gamma & $\mathrm{N}$ \\
\hline \hline$<0.94$ & 0.637 & 0.079 & 0.310 & 0.148 & 0.117 & 0.545 & 2 \\
$0.94-0.97$ & 0.855 & 0.238 & 0.683 & 0.334 & 0.329 & 0.643 & 40 \\
$0.97-1.00$ & 1.044 & 0.531 & 0.783 & 0.581 & 0.534 & 0.998 & 91 \\
$1.00-1.03$ & 0.836 & 0.721 & 0.751 & 0.752 & 0.848 & 0.815 & 107 \\
$1.03-1.06$ & 1.035 & 1.131 & 0.670 & 0.732 & 0.687 & 0.893 & 108 \\
$>1.06$ & 1.064 & 5.911 & 0.882 & 0.823 & 0.847 & 0.860 & 263 \\
Total & 1.005 & 3.925 & 0.797 & 0.737 & 0.753 & 0.867 & 611 \\
\hline
\end{tabular}

(c) In-sample RMSE for the long maturity group with time-varying parameters.

\begin{tabular}{cccccccc}
\hline Moneyness & BS & Pr. BS & GC $^{+}$ & $\mathrm{SNP}(\mathrm{m}=2)$ & G. Beta & V. Gamma & $\mathrm{N}$ \\
\hline \hline$<0.94$ & 1.878 & 0.330 & 0.848 & 0.251 & 0.169 & 0.141 & 360 \\
$0.94-0.97$ & 1.634 & 0.298 & 0.625 & 0.191 & 0.149 & 0.145 & 365 \\
$0.97-1.00$ & 1.196 & 0.251 & 0.366 & 0.175 & 0.195 & 0.161 & 457 \\
$1.00-1.03$ & 0.630 & 0.209 & 0.323 & 0.202 & 0.188 & 0.144 & 474 \\
$1.03-1.06$ & 0.968 & 0.244 & 0.454 & 0.166 & 0.174 & 0.152 & 440 \\
$>1.06$ & 1.662 & 0.393 & 0.447 & 0.277 & 0.324 & 0.254 & 1,599 \\
Total & 1.464 & 0.327 & 0.500 & 0.235 & 0.251 & 0.201 & 3,695 \\
\hline
\end{tabular}

(d) Out-of-sample RMSE for the long maturity group with time-varying parameters.

\begin{tabular}{cccccccc}
\hline Moneyness & BS & Pr. BS & GC $^{+}$ & $\mathrm{SNP}(\mathrm{m}=2)$ & G. Beta & V. Gamma & $\mathrm{N}$ \\
\hline \hline$<0.94$ & 2.045 & 0.401 & 0.832 & 0.596 & 0.324 & 0.605 & 36 \\
$0.94-0.97$ & 2.153 & 0.929 & 0.935 & 0.900 & 0.692 & 1.126 & 59 \\
$0.97-1.00$ & 1.654 & 0.977 & 0.987 & 0.894 & 0.876 & 0.813 & 66 \\
$1.00-1.03$ & 1.102 & 0.840 & 1.077 & 1.079 & 1.020 & 0.797 & 94 \\
$1.03-1.06$ & 1.358 & 0.764 & 0.969 & 0.953 & 0.943 & 0.792 & 97 \\
$>1.06$ & 1.838 & 1.985 & 0.906 & 1.155 & 0.893 & 0.916 & 259 \\
Total & 1.703 & 1.438 & 0.952 & 1.037 & 0.880 & 0.876 & 611 \\
\hline
\end{tabular}

Notes: In-sample analysis uses different parameters for each Wednesday from 1988 to 1992, while Outof-sample tables use the parameters estimated on the previous Wednesday during 1993. Moneyness is defined as the ratio of the implicit forward price of the underlying asset to the strike price. BS, Pr. BS, $\mathrm{GC}^{+}$, G. Beta and V. Gamma denote, respectively, Black-Scholes, Practitioners' Black-Scholes, Gram-Charlier with positivity restrictions, Generalised Beta and Variance Gamma models. $N$ denotes the number of option prices per moneyness category. 
Table 2

(a) In-sample RMSE for the short maturity group with fixed shape parameters.

\begin{tabular}{cccccccccc}
\hline Moneyness & \multicolumn{3}{c}{ Prac. } & \multicolumn{3}{c}{$\mathrm{SNP}$} & \multicolumn{3}{c}{ Gen. } \\
& $\mathrm{BS}$ & $\mathrm{BS}$ & $\mathrm{GC}^{+}$ & $\mathrm{m}=2$ & $\mathrm{~m}=3$ & $\mathrm{~m}=4$ & Beta & Gamma & $\mathrm{N}$ \\
\hline \hline$<0.94$ & 0.488 & 0.211 & 0.228 & 0.230 & 0.206 & 0.193 & 0.215 & 0.285 & 65 \\
$0.94-0.97$ & 0.542 & 0.296 & 0.235 & 0.256 & 0.242 & 0.236 & 0.221 & 0.237 & 287 \\
$0.97-1.00$ & 0.489 & 0.285 & 0.250 & 0.246 & 0.243 & 0.241 & 0.260 & 0.277 & 450 \\
$1.00-1.03$ & 0.291 & 0.213 & 0.202 & 0.206 & 0.196 & 0.191 & 0.222 & 0.221 & 439 \\
$1.03-1.06$ & 0.662 & 0.295 & 0.283 & 0.292 & 0.282 & 0.278 & 0.262 & 0.270 & 434 \\
$>1.06$ & 0.732 & 0.503 & 0.473 & 0.443 & 0.422 & 0.408 & 0.464 & 0.451 & 1,176 \\
Total & 0.611 & 0.384 & 0.357 & 0.343 & 0.328 & 0.319 & 0.351 & 0.349 & 2,851 \\
\hline
\end{tabular}

(b) Out-of-sample RMSE for the short maturity group with fixed shape parameters.

\begin{tabular}{cccccccccc}
\hline Moneyness & \multicolumn{3}{c}{ Prac. } & \multicolumn{3}{c}{$\mathrm{SNP}$} & \multicolumn{3}{c}{ Gen. } \\
& $\mathrm{BS}$ & $\mathrm{BS}$ & $\mathrm{GC}^{+}$ & $\mathrm{m}=2$ & $\mathrm{~m}=3$ & $\mathrm{~m}=4$ & Beta & Gamma & $\mathrm{N}$ \\
\hline \hline$<0.94$ & 0.637 & 0.086 & 0.232 & 0.132 & 0.110 & 0.097 & 0.241 & 0.316 & 2 \\
$0.94-0.97$ & 0.855 & 0.286 & 0.410 & 0.427 & 0.391 & 0.367 & 0.387 & 0.451 & 40 \\
$0.97-1.00$ & 1.044 & 0.715 & 0.668 & 0.695 & 0.678 & 0.660 & 0.642 & 0.620 & 91 \\
$1.00-1.03$ & 0.836 & 0.739 & 0.723 & 0.719 & 0.723 & 0.720 & 0.762 & 0.757 & 107 \\
$1.03-1.06$ & 1.035 & 0.694 & 0.637 & 0.632 & 0.630 & 0.627 & 0.652 & 0.644 & 108 \\
$>1.06$ & 1.064 & 0.859 & 0.882 & 0.815 & 0.775 & 0.740 & 0.862 & 0.846 & 263 \\
Total & 1.005 & 0.762 & 0.759 & 0.729 & 0.706 & 0.685 & 0.754 & 0.743 & 611 \\
\hline
\end{tabular}

(c) In-sample RMSE for the long maturity group with fixed shape parameters.

\begin{tabular}{cccccccccc}
\hline Moneyness & \multicolumn{3}{c}{ Prac. } & \multicolumn{3}{c}{$\mathrm{SNP}$} & \multicolumn{3}{c}{ Gen. } \\
& $\mathrm{BS}$ & $\mathrm{BS}$ & $\mathrm{GC}^{+}$ & $\mathrm{m}=2$ & $\mathrm{~m}=3$ & $\mathrm{~m}=4$ & Beta & Gamma & $\mathrm{N}$ \\
\hline \hline$<0.94$ & 1.878 & 0.582 & 0.851 & 0.554 & 0.551 & 0.508 & 0.496 & 0.497 & 360 \\
$0.94-0.97$ & 1.634 & 0.521 & 0.637 & 0.450 & 0.438 & 0.438 & 0.444 & 0.450 & 365 \\
$0.97-1.00$ & 1.196 & 0.399 & 0.406 & 0.349 & 0.338 & 0.337 & 0.338 & 0.339 & 457 \\
$1.00-1.03$ & 0.630 & 0.256 & 0.342 & 0.252 & 0.218 & 0.217 & 0.218 & 0.223 & 474 \\
$1.03-1.06$ & 0.968 & 0.302 & 0.448 & 0.250 & 0.230 & 0.230 & 0.224 & 0.225 & 440 \\
$>1.06$ & 1.662 & 0.583 & 0.530 & 0.512 & 0.461 & 0.455 & 0.453 & 0.454 & 1,599 \\
Total & 1.464 & 0.496 & 0.540 & 0.441 & 0.404 & 0.400 & 0.398 & 0.400 & 3,695 \\
\hline
\end{tabular}

(d) Out-of-sample RMSE for the long maturity group with fixed shape parameters.

\begin{tabular}{cccccccccc}
\hline Moneyness & \multicolumn{3}{c}{ Prac. } & \multicolumn{3}{c}{$\mathrm{SNP}$} & \multicolumn{3}{c}{ Gen. } \\
& $\mathrm{BS}$ & $\mathrm{BS}$ & $\mathrm{GC}^{+}$ & $\mathrm{m}=2$ & $\mathrm{~m}=3$ & $\mathrm{~m}=4$ & Beta & Gamma & $\mathrm{N}$ \\
\hline \hline$<0.94$ & 2.045 & 0.585 & 0.765 & 0.420 & 0.328 & 0.319 & 0.366 & 0.404 & 36 \\
$0.94-0.97$ & 2.153 & 1.039 & 0.899 & 0.720 & 0.707 & 0.711 & 0.706 & 0.701 & 59 \\
$0.97-1.00$ & 1.654 & 1.081 & 1.012 & 0.988 & 0.996 & 1.001 & 0.995 & 0.992 & 66 \\
$1.00-1.03$ & 1.102 & 0.765 & 1.046 & 0.989 & 0.980 & 0.982 & 0.976 & 0.972 & 94 \\
$1.03-1.06$ & 1.358 & 0.678 & 0.923 & 0.882 & 0.883 & 0.886 & 0.886 & 0.884 & 97 \\
$>1.06$ & 1.838 & 0.613 & 0.849 & 0.903 & 0.841 & 0.832 & 0.841 & 0.847 & 259 \\
Total & 1.703 & 0.757 & 0.912 & 0.886 & 0.856 & 0.854 & 0.857 & 0.858 & 611 \\
\hline
\end{tabular}

Notes: In-sample analysis (1988 to 1992) allows volatility to be time varying, but the other shape parameters are kept fixed. Out-of-sample estimates (1993) use for each week the volatility from the previous week and the fixed shape parameters estimated from the first five years. Moneyness is the ratio of the implicit forward price to the strike price. BS, Pr. BS, $\mathrm{GC}^{+}$, G. Beta and V. Gamma denote, respectively, Black-Scholes, Practitioners' Black-Scholes, Gram-Charlier with positivity restrictions, Generalised Beta and Variance Gamma models. $N$ denotes the number of option prices per moneyness category. 
Figure 1

Regions of skewness and kurtosis

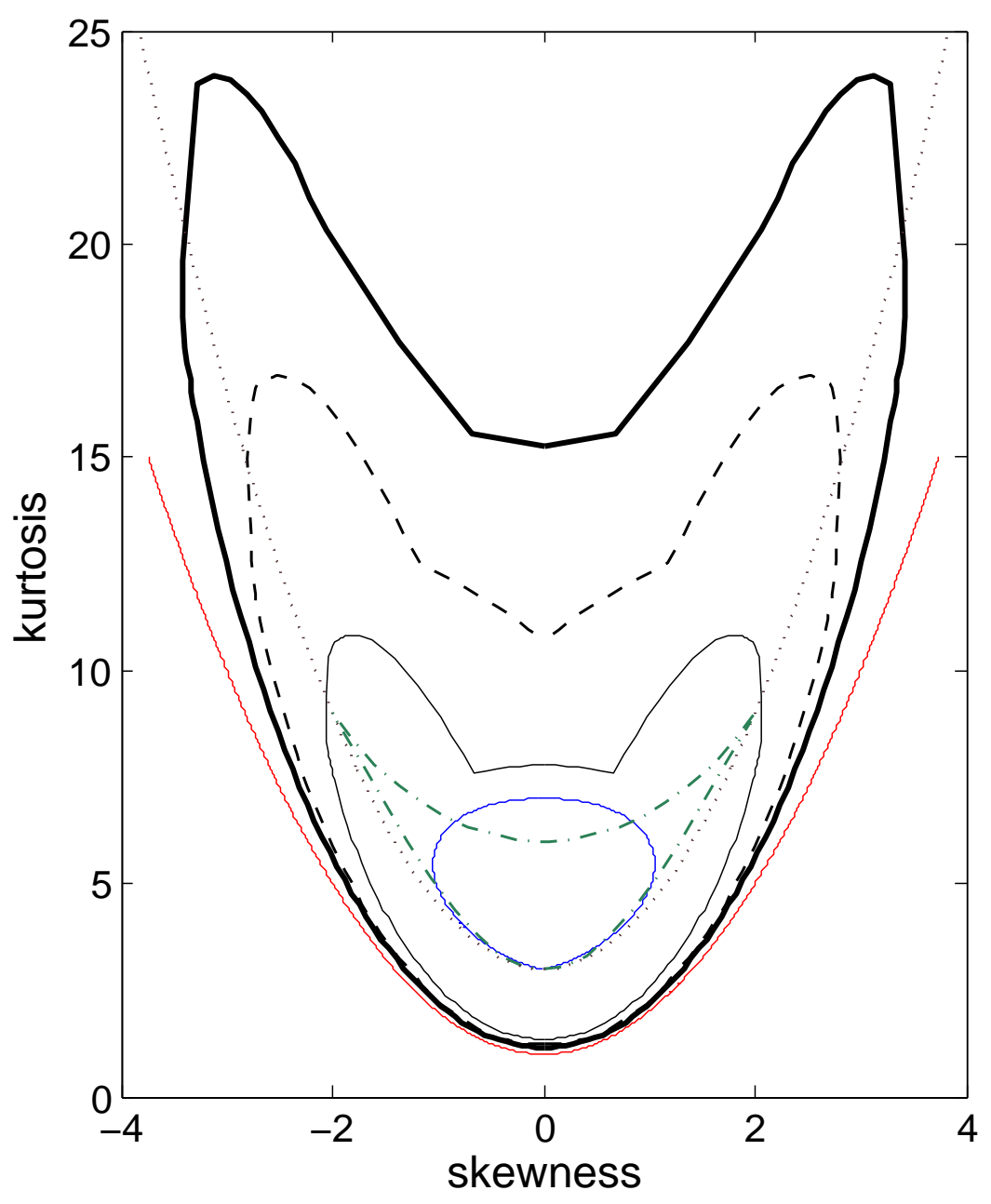

\begin{tabular}{|c|}
\hline $\mathrm{GC}^{+}$ \\
$-\mathrm{SNP} \mathrm{m}=2$ \\
$---\mathrm{SNP} \mathrm{m}=3$ \\
- SNP $\mathrm{m}=4$ \\
$\cdots$ Var. Gamma \\
- Gen. Beta \\
- Frontier
\end{tabular}

Note: $\mathrm{GC}^{+}$denotes a Gram-Charlier expansion of order $n=4$ with positivity restrictions, while Gen. Beta denotes the distribution of the log of a Generalised Beta. 
Figure 2

Flexibility to model departures from Black-Scholes

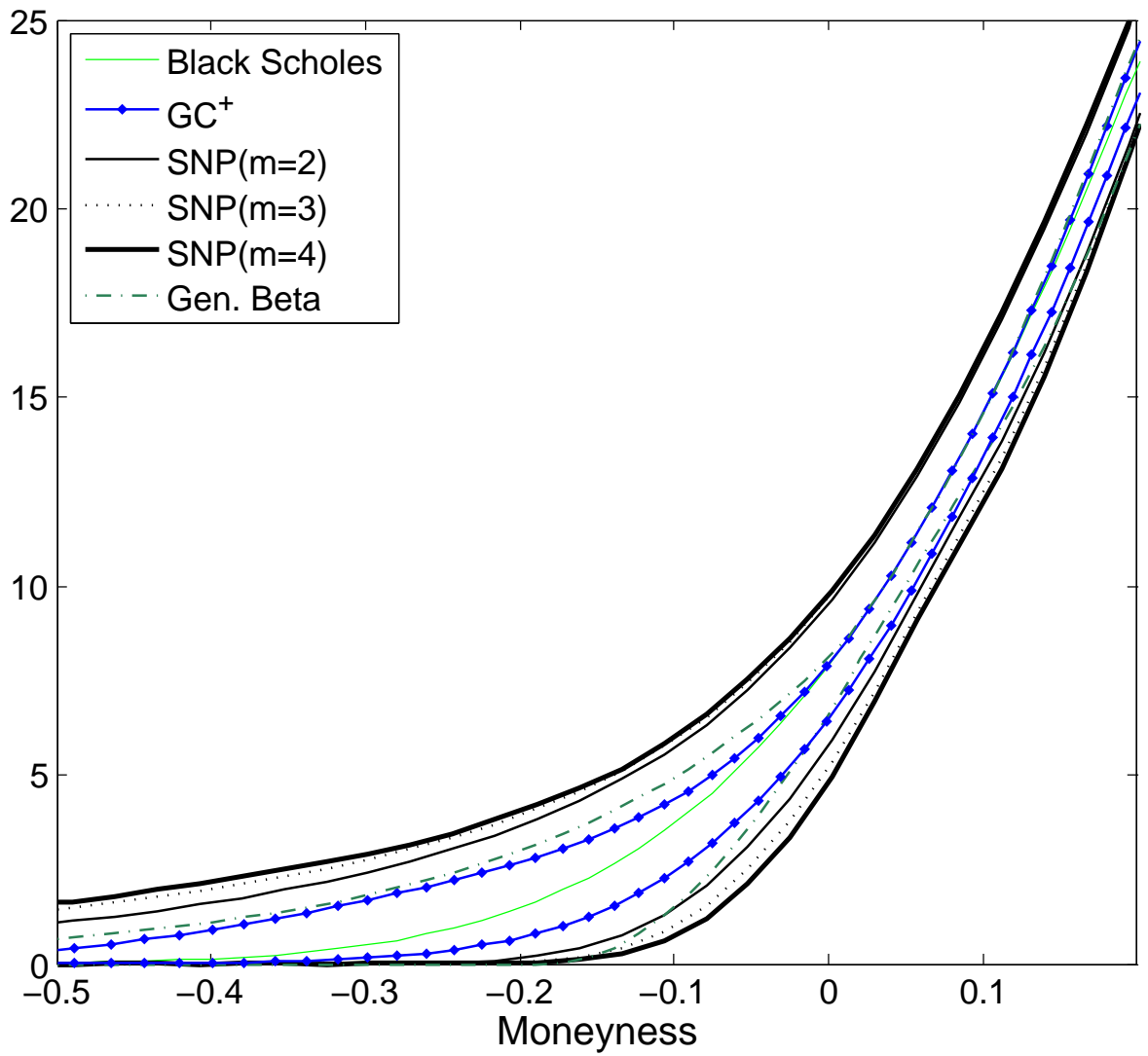

Note: This figure shows the minimum and maximum European call prices that each distribution can yield for a strike price of 100 , a maturity of 3 months and a risk free interest rate of $3 \%$. $\mathrm{GC}^{+}$ denotes a Gram-Charlier expansion of order $n=4$ with positivity restrictions. 
Figure 3a: Estimation of options from Marron-Wand test suite

Density

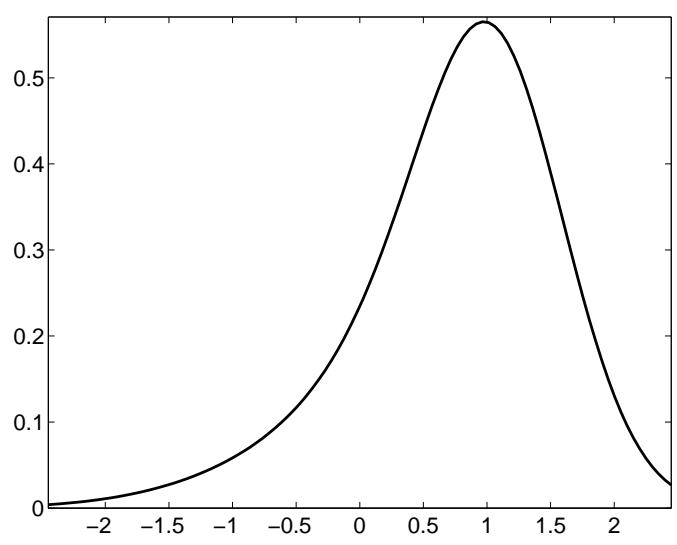

Implied volatilities

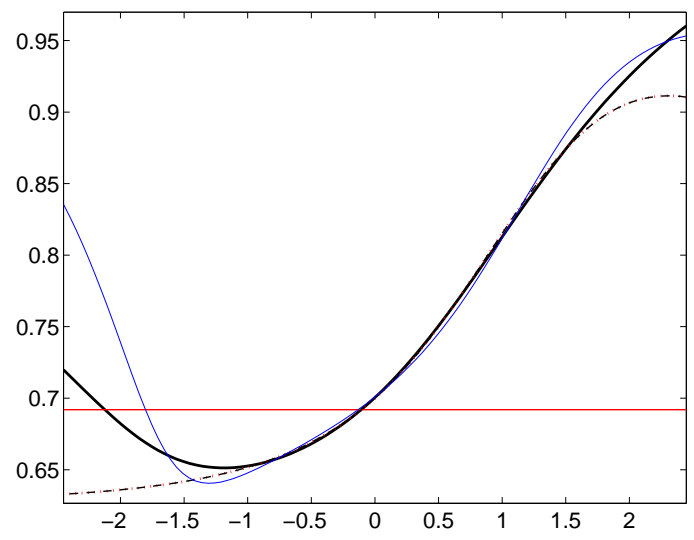

Strongly skewed $\left(m^{*}=12\right)$

Density

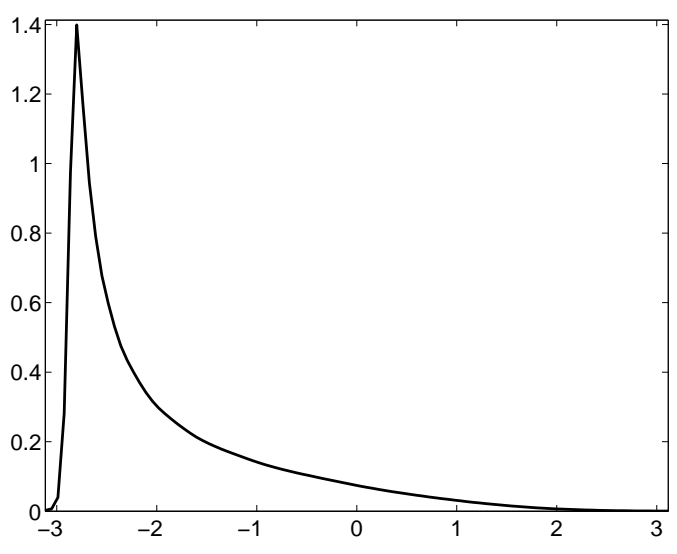

Implied volatilities

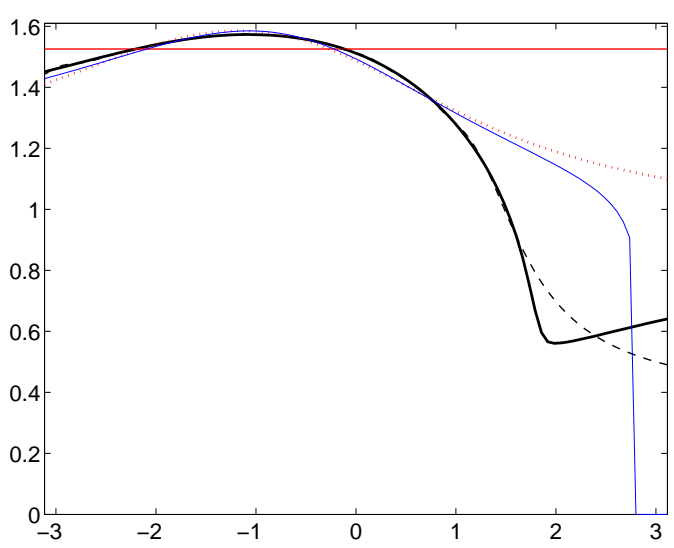

Kurtotic unimodal $\left(m^{*}=13\right)$

Density

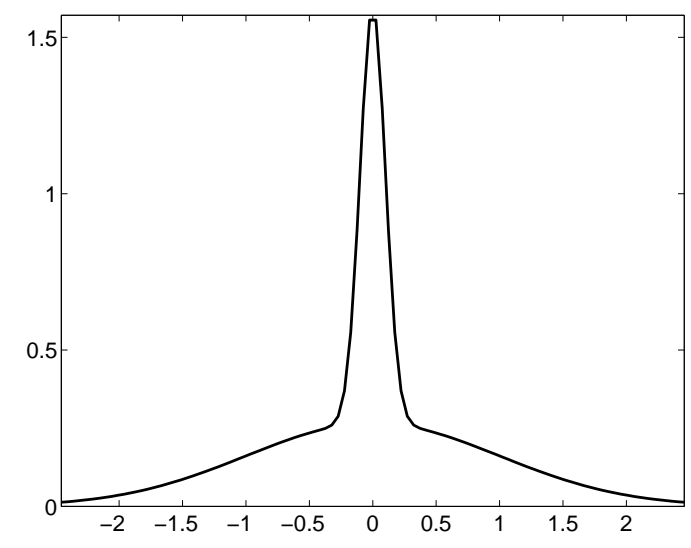

Implied volatilities

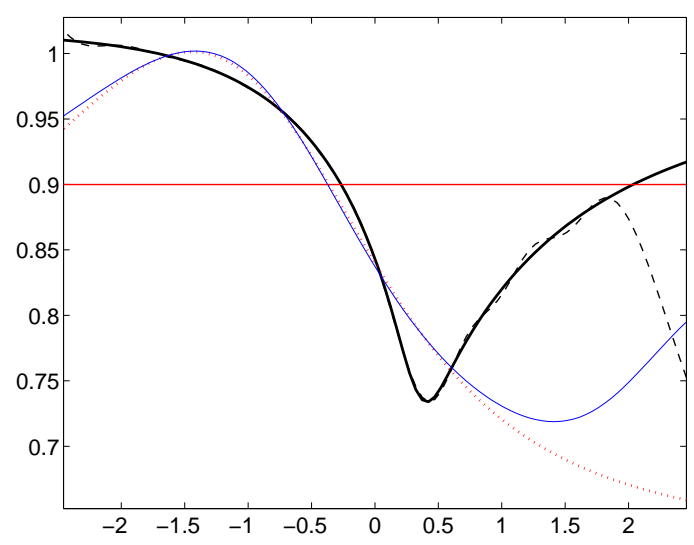

GC

Notes: Marron-Wand densities are represented in the left panels. The corresponding true implied volatilities are plotted on the right panels, together with the ones obtained by estimating the SNP and Gram-Charlier option pricing models. SNP $\left(m^{*}\right)$ denotes the SNP model of lowest order that makes the root mean square pricing error divided by the mean call price smaller than 10 basis point. The remaining non-Gaussian models only use two shape parameters. 
Figure 3b: Estimation of options from Marron-Wand test suite

Density

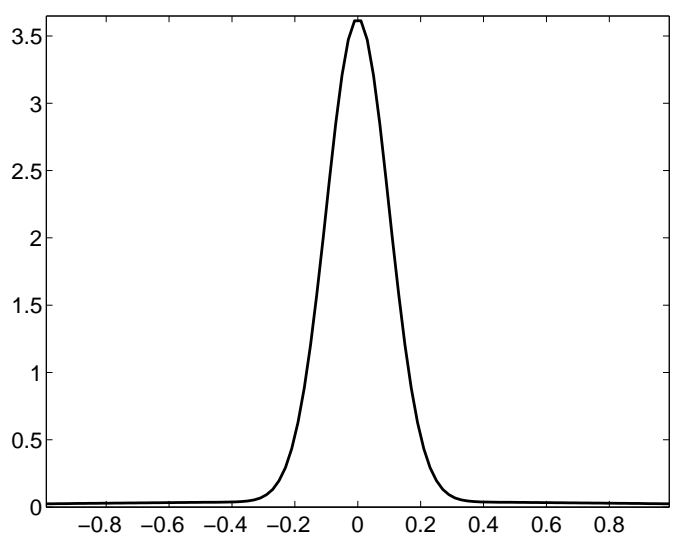

Outlier $\left(m^{*}=8\right)$

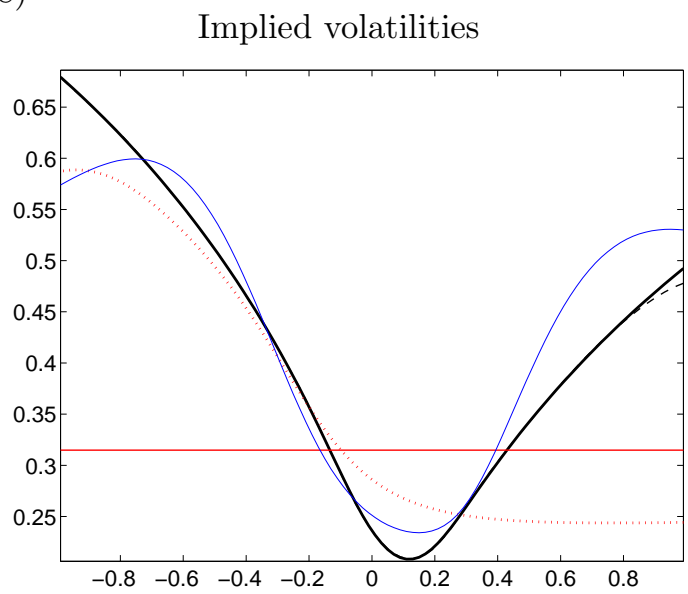

Density

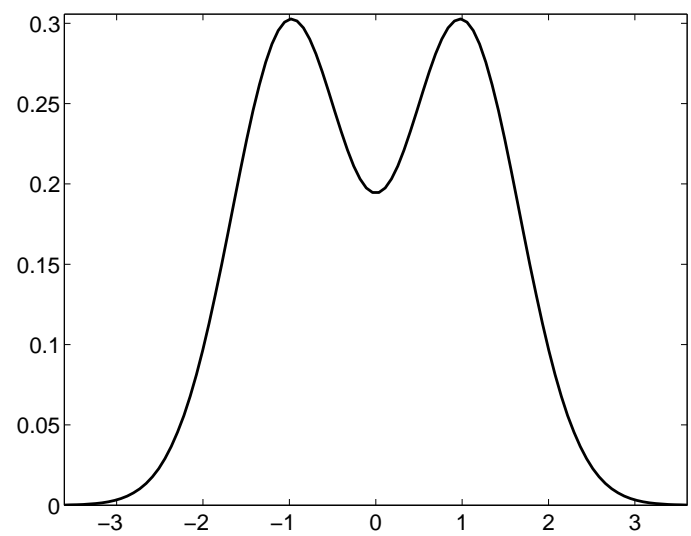

Bimodal $\left(m^{*}=2\right)$

\section{Density}

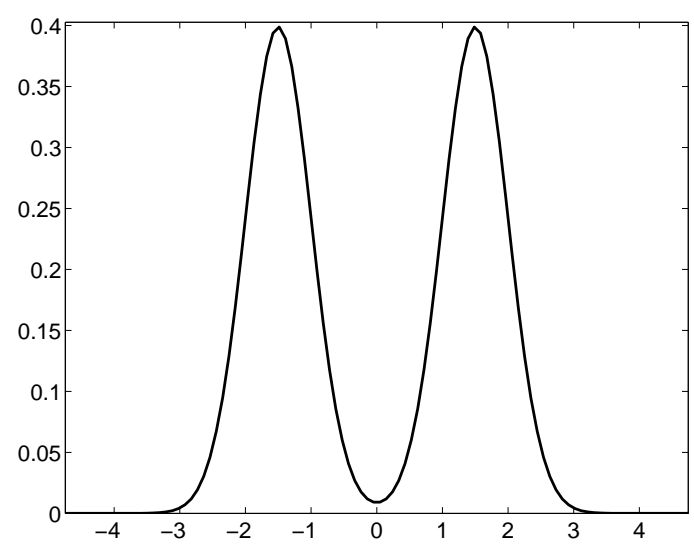

Implied volatilities

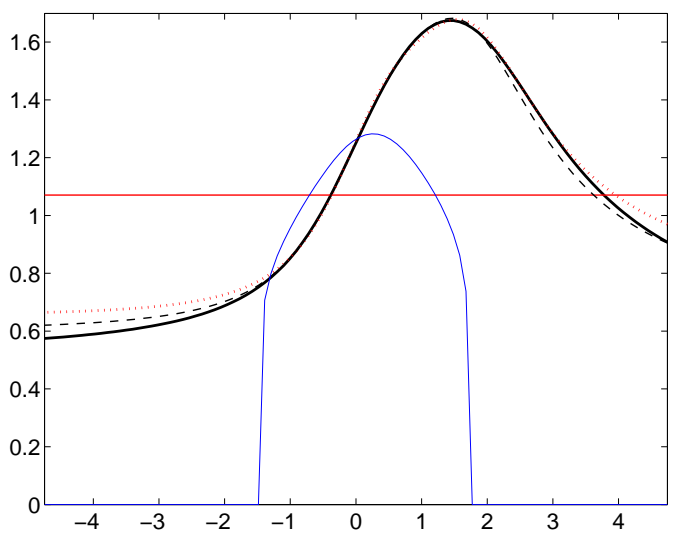

GC

Notes: Marron-Wand densities are represented in the left panels. The corresponding true implied volatilities are plotted on the right panels, together with the ones obtained by estimating the SNP and Gram-Charlier (GC) option pricing models. SNP $\left(m^{*}\right)$ denotes the SNP model of lowest order that makes the root mean square pricing error divided by the mean call price smaller than 10 basis point. The remaining non-Gaussian models only use two shape parameters. 
Figure 3c: Estimation of options from Marron-Wand test suite

Density

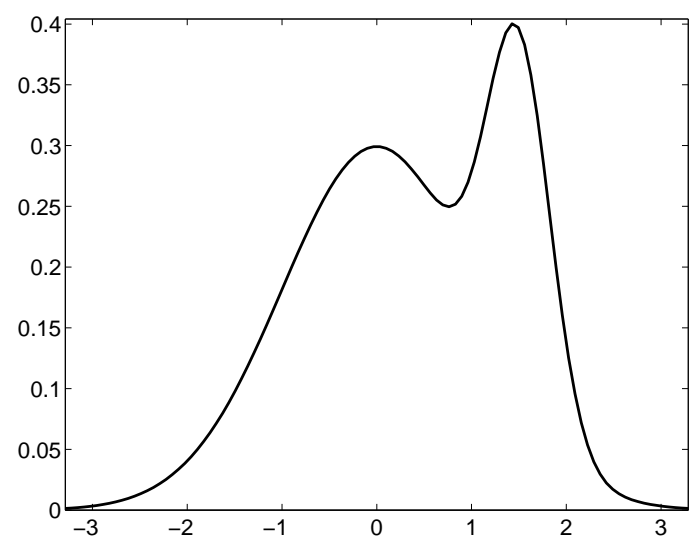

Implied volatilities

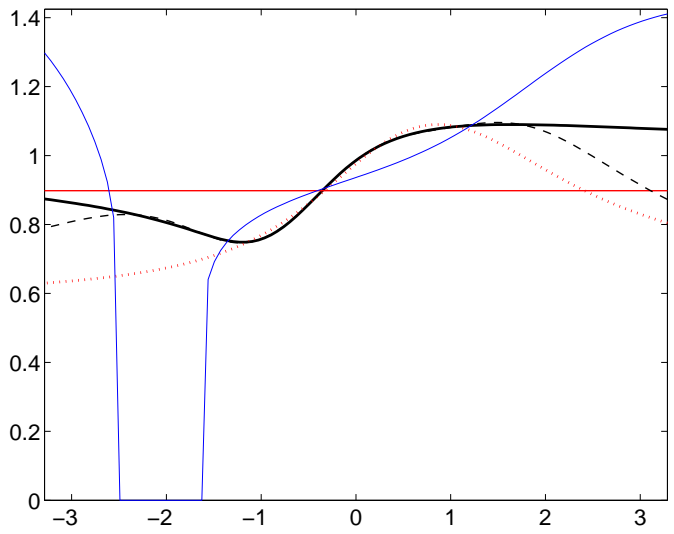

Density

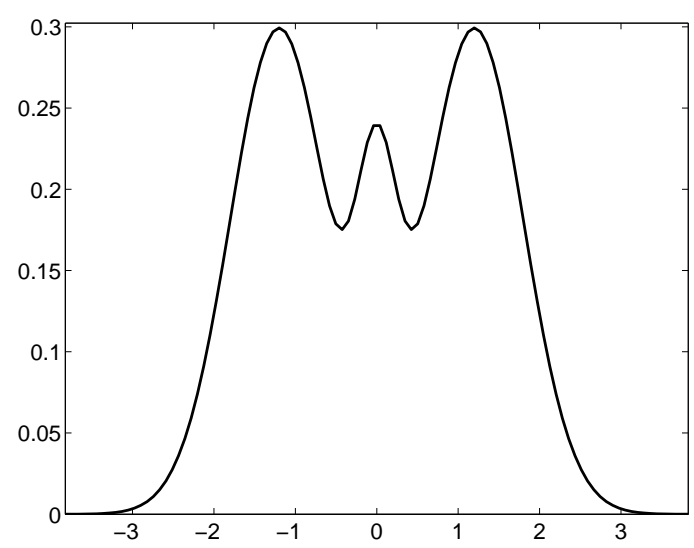

Implied volatilities

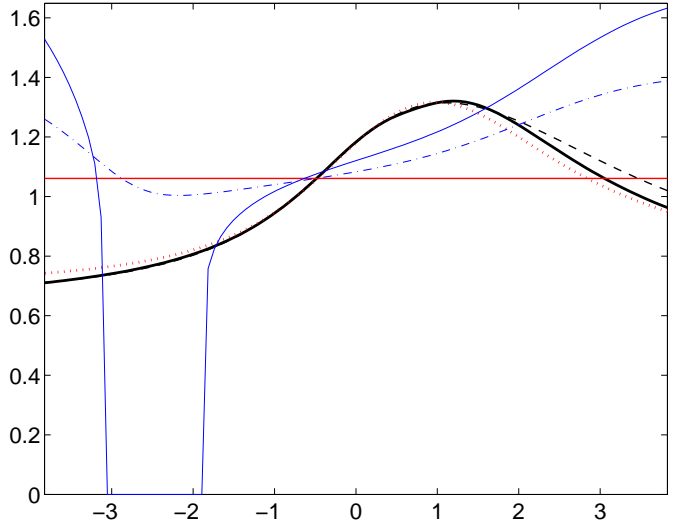

Density

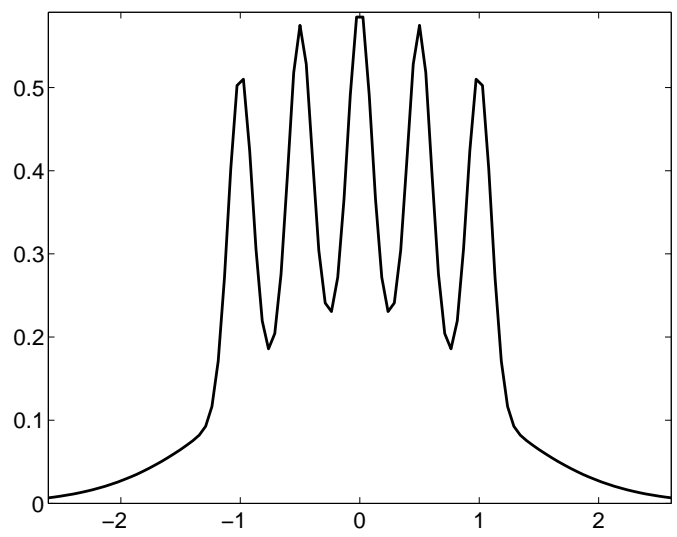

Implied volatilities

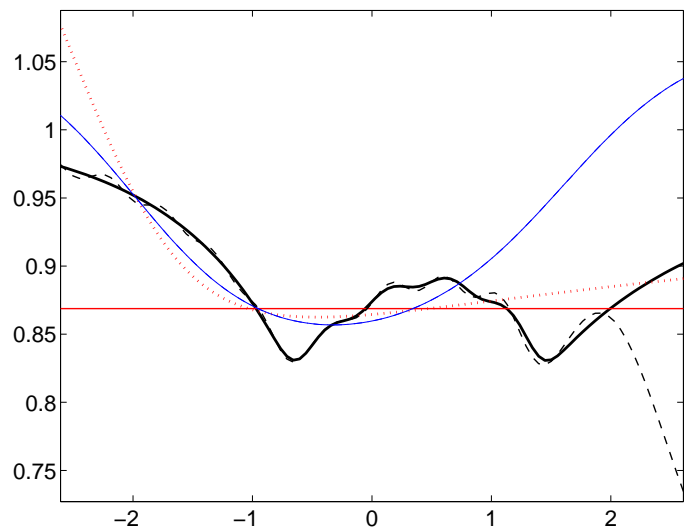

$\mathrm{GC}$

Notes: Marron-Wand densities are represented in the left panels. The corresponding true implied volatilities are plotted on the right panels, together with the ones obtained by estimating the SNP and Gram-Charlier (GC) option pricing models. SNP $\left(m^{*}\right)$ denotes the SNP model of lowest order that makes the root mean square pricing error divided by the mean call price smaller than 10 basis point. The remaining non-Gaussian models only use two shape parameters. 
Figure 4: Fit of the implied volatility of a multiperiod SNP process

(a) One-month options $\left(m^{*}=4\right)$

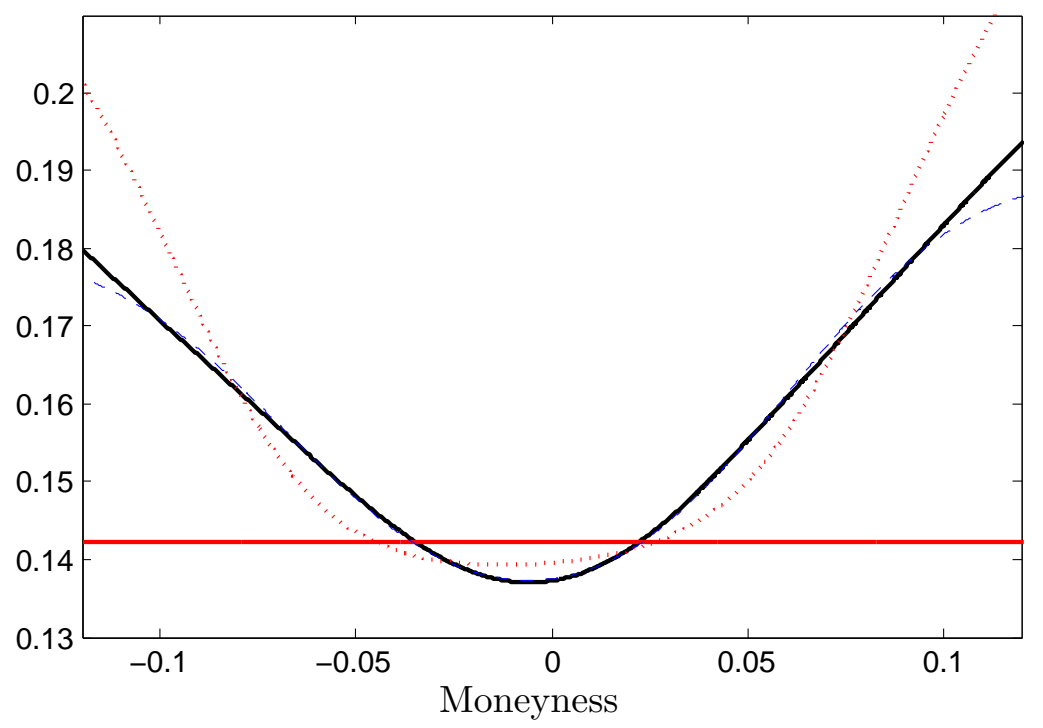

(b) Three-month options $\left(m^{*}=4\right)$

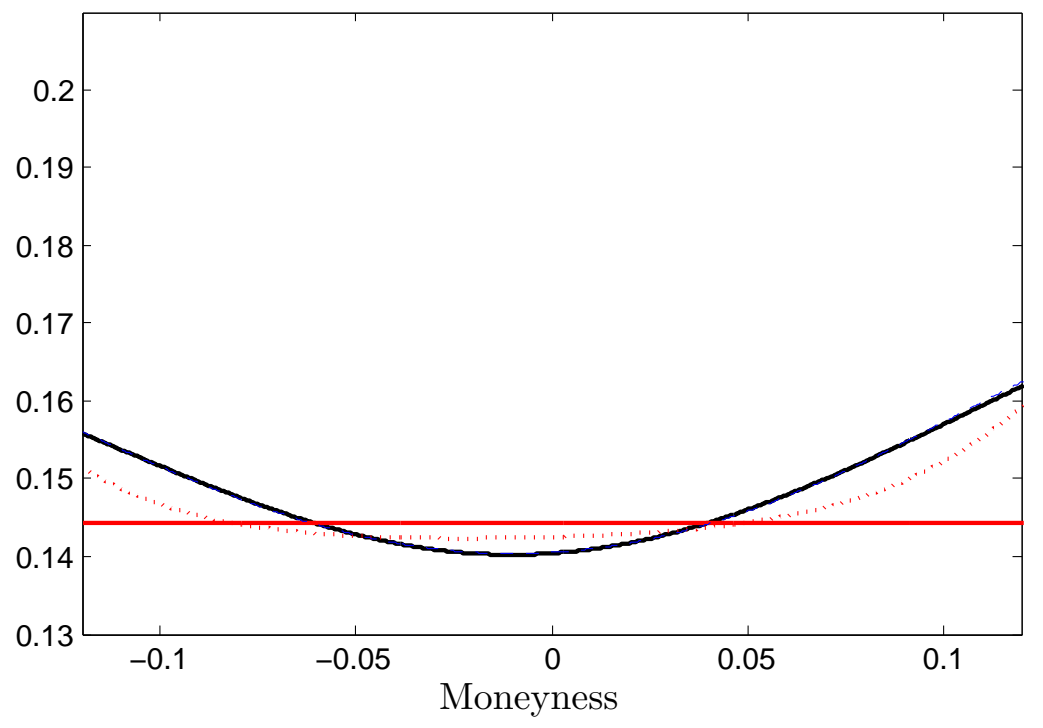

\begin{tabular}{|l|} 
- High Frequency SNP \\
BS \\
$\cdots \cdots \cdot \operatorname{SNP}(m=2)$ \\
$---\operatorname{SNP}\left(m^{*}\right)$
\end{tabular}

Notes: SNP $\left(m^{*}\right)$ denotes the SNP model of lowest order that makes the root mean square pricing error divided by the mean call price smaller than 10 basis point. The option prices of the high frequency SNP model have been generated by assuming that the weekly log-returns under the risk-neutral measure are SNP of order 2 whose skewness and kurtosis is -0.4 and 6.5 , respectively. Finally, the volatility follows a Markov chain with two states: $\sigma_{1}=0.1960$ and $\sigma_{2}=0.1023$. The probabilities of remaining in states 1 and 2 are $p=0.9787$ and $q=0.9847$, respectively. The risk-free rate is set at $r=3 \%$. 
Figure 5a

Skewness and kurtosis for the short maturity group with time-varying parameters
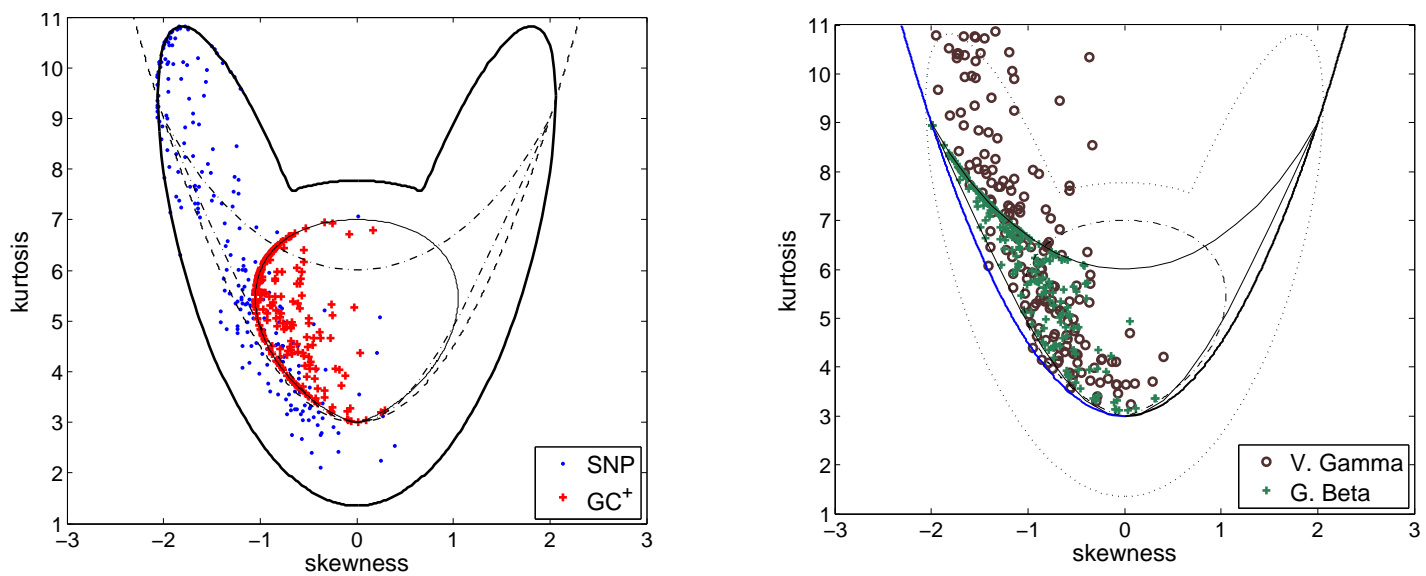

Figure 5b

Skewness and kurtosis for the long maturity group with time-varying parameters

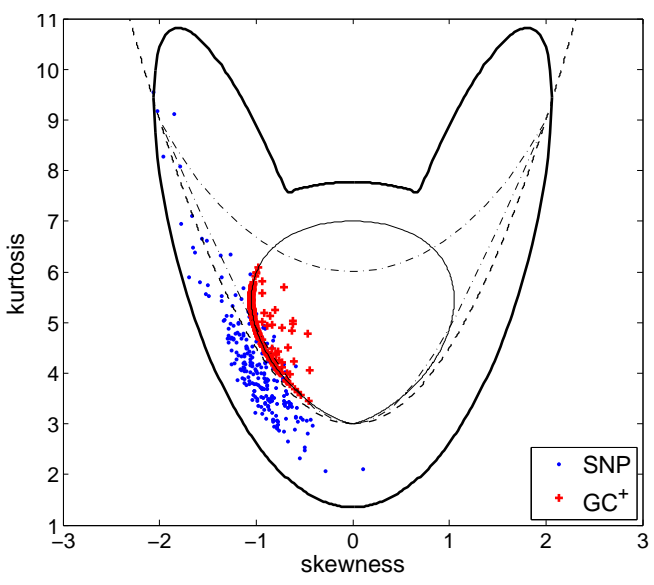

Figure 5c

Skewness and kurtosis of the

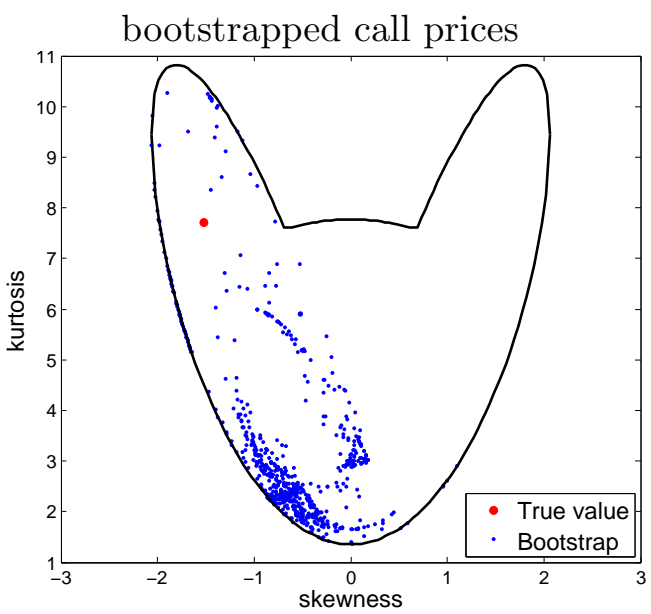

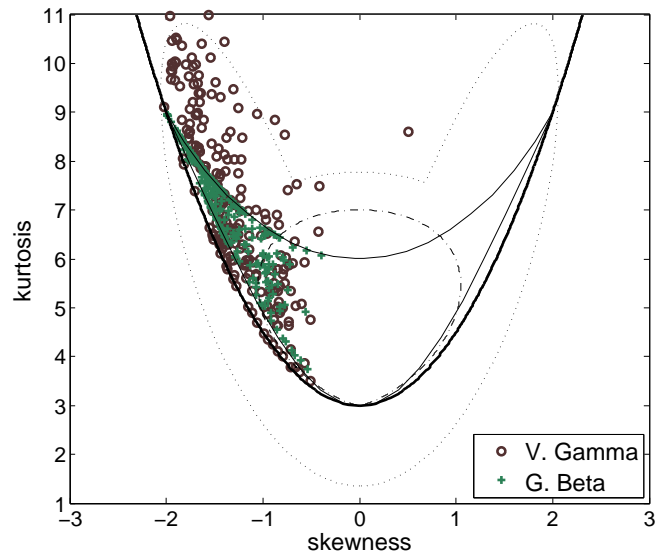

Figure 5d Skewness and kurtosis for fixed parameters

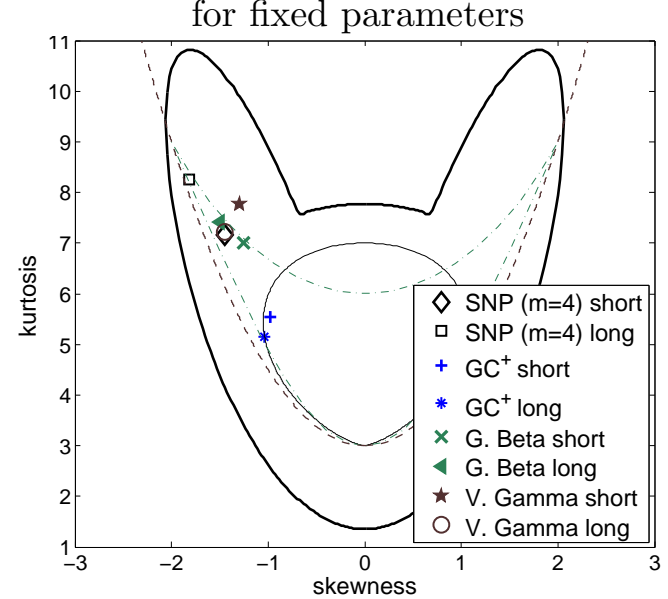

Notes: The results in Figures $5 \mathrm{a}$ and $5 \mathrm{~b}$ correspond to separate estimations for each Wednesday in-sample, while to obtain Figure $5 \mathrm{~d}$ all parameters except volatility are assumed to be constant over the whole sample. In Figures $5 \mathrm{a}$ and 5b SNP refers to a semi-nonparametric distribution of order 2. $\mathrm{GC}^{+}$, G. Beta and V. Gamma denote, respectively, the Gram-Charlier expansion $(n=4)$ with positivity restrictions, the Generalised Beta and Variance Gamma models, while "Short" and "Long" denote the short and long maturity groups. 
Figure 6

Volatility estimates for the short maturities

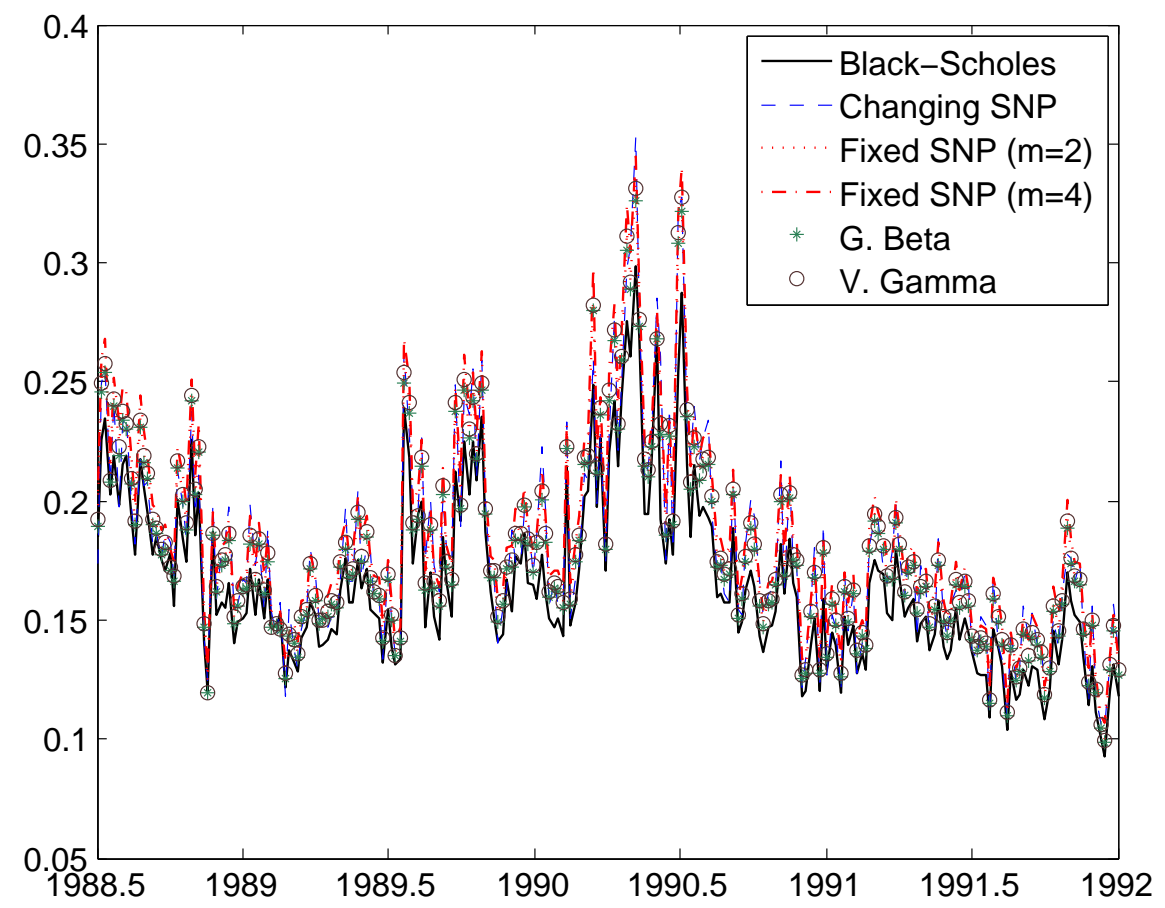

Note: "Fixed SNP" assumes that the shape parameters of the SNP are constant over time, while "Changing SNP" allows them to be time varying. Gen. Beta and V. Gamma denote, respectively, the Generalised Beta and Variance Gamma models. 
Figure $7 \mathrm{a}$

Risk-neutral density of $\log \left(S_{T} / S_{t}\right)$ for the short maturity group

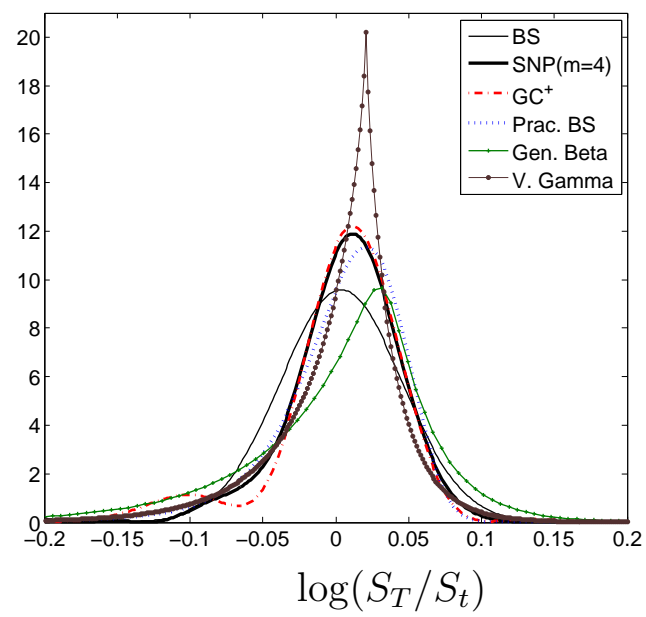

Figure 7c

Risk-neutral density of $\log \left(S_{T} / S_{t}\right)$ for the long maturity group

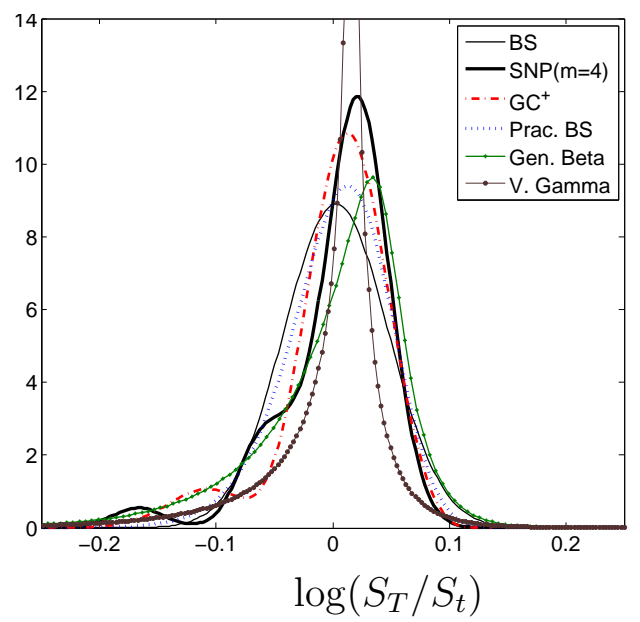

Figure $7 \mathrm{~b}$

Left tail of the risk-neutral density of $\log \left(S_{T} / S_{t}\right)$ for the short maturity group

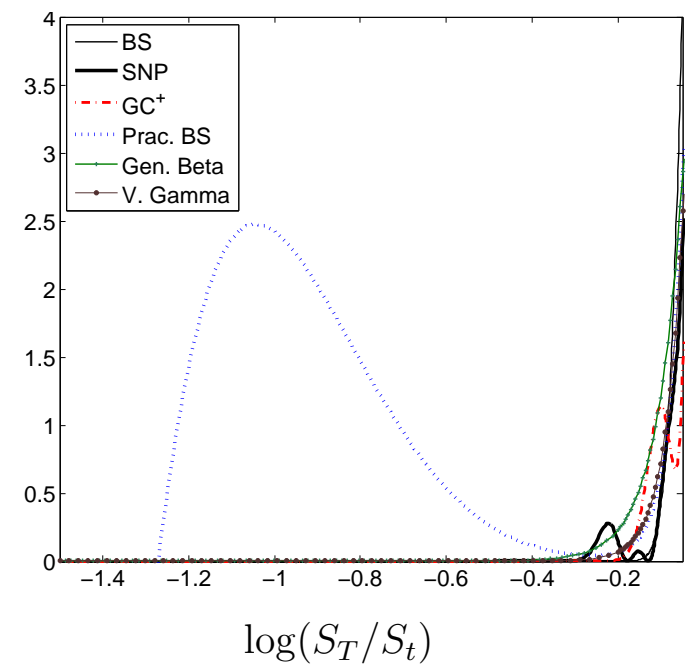

Figure $7 \mathrm{~d}$

Left tail of the risk-neutral density of $\log \left(S_{T} / S_{t}\right)$ for the long maturity group

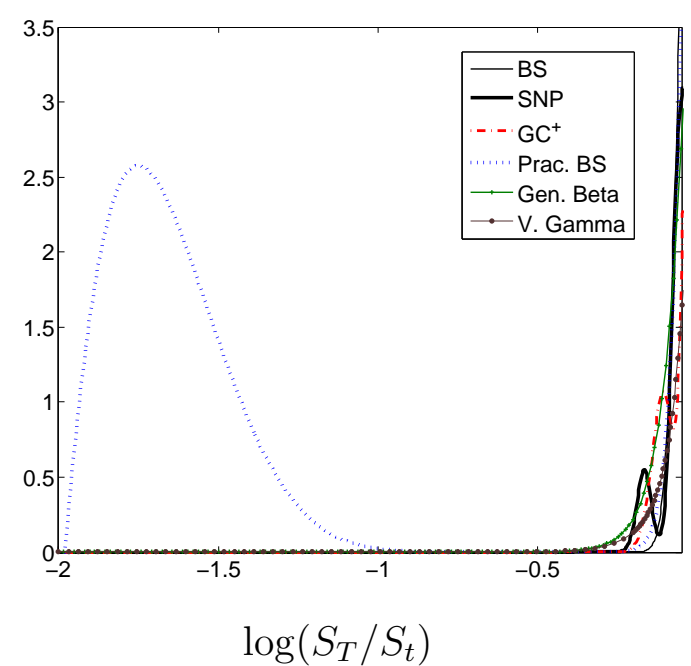

Notes: These results are based on the volatility estimated on November 13, 1991, but the shape parameters are estimated using data between 1988 and 1992. Pract. BS denotes a model in which volatility is a quadratic function of moneyness. SNP refers to a seminonparametric distribution of order 4, while $\mathrm{GC}^{+}$, Gen. Beta and V. Gamma denote, respectively, the Gram-Charlier distribution $(n=4)$ with positivity restrictions, the Generalised Beta and Variance Gamma models. 
Figure 8:

Implied volatility on November 13, 1991

(a) Short maturity group

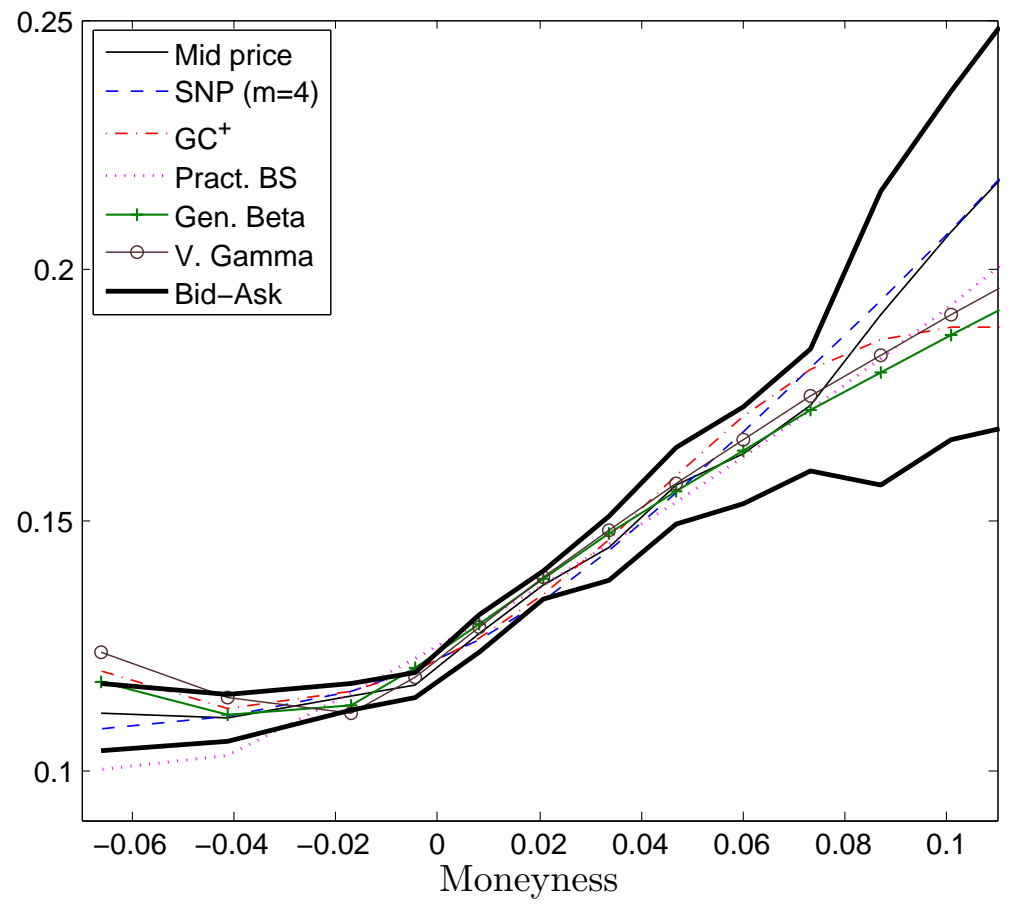

(b) Long maturity group

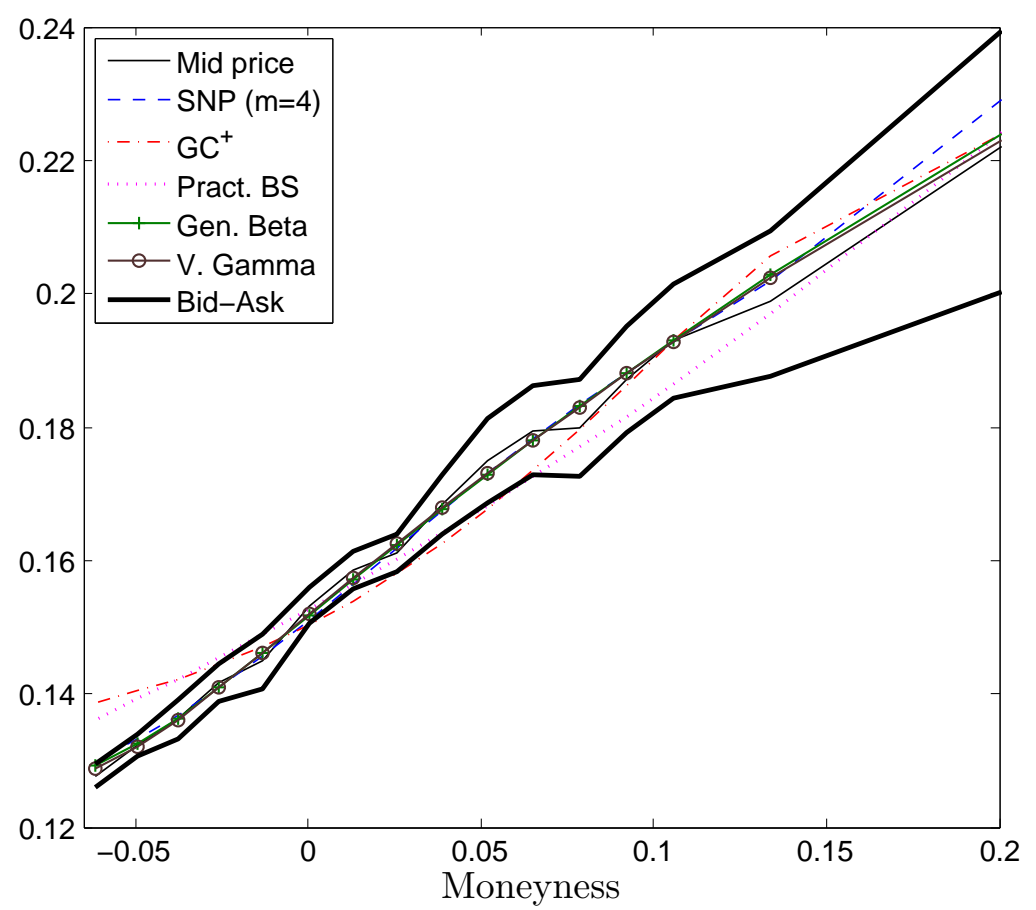

Note: All the models use time varying volatilities but constant shape parameters. Moneyness defined as $\log \left(S_{t} / K\right)+r(T-t)$. Pract. BS denotes a model in which volatility is a quadratic function of moneyness. SNP $(\mathrm{m}=4)$ refers to a seminonparametric distribution of order 4 , while $\mathrm{GC}^{+}$, Gen. Beta and V. Gamma denote, respectively, the Gram-Charlier expansion $(n=4)$ with positivity restrictions, the Generalised Beta and Variance Gamma models. 


\title{
Supplemental appendices for Parametric properties of semi-nonparametric distributions, with applications to option valuation
}

\author{
Ángel León \\ Universidad de Alicante \\ Javier Mencía \\ $<$ aleon@ua.es> \\ Bank of Spain \\ $<$ javier.mencia@bde.es> \\ Enrique Sentana \\ CEMFI \\ $<$ sentana@cemfi.es $>$ \\ June 2004 \\ Revised: February 2007
}




\section{B Properties of Hermite polynomials}

The $j^{\text {th }}$ derivative of a Hermite polynomial of order $k$ (see Stuart and Ord, 1977), is

$$
\frac{d^{j}}{d x^{j}} H_{k}(x)=\sqrt{\frac{k !}{(k-j) !}} H_{k-j}(x)
$$

if $j \leq k$, and zero otherwise. Using this result, $H_{k}(a+b)$ can be expressed as the following finite order Taylor expansion around $a$

$$
\begin{aligned}
H_{k}(a+b) & =\left.\sum_{j=0}^{k} \frac{1}{j !} \frac{d^{j}}{d x^{j}} H_{k}(x)\right|_{x=a} b^{j} \\
& =\sum_{j=0}^{k} \frac{1}{j !} \sqrt{\frac{k !}{(k-j) !}} H_{k-j}(a) b^{j}
\end{aligned}
$$

\section{Proofs}

\section{Proposition 1}

We know that

$$
\frac{1}{\boldsymbol{\nu}^{\prime} \boldsymbol{\nu}}\left[\sum_{i=0}^{m} \nu_{i} H_{i}(x)\right]^{2}=\sum_{i=0}^{m} \sum_{j=0}^{m} \frac{\nu_{i} \nu_{j}}{\boldsymbol{\nu}^{\prime} \boldsymbol{\nu}} H_{i}(x) H_{j}(x)=\sum_{k=0}^{2 m} \gamma_{k}(\boldsymbol{\nu}) H_{k}(x),
$$

where it is verified that $\forall i, j$

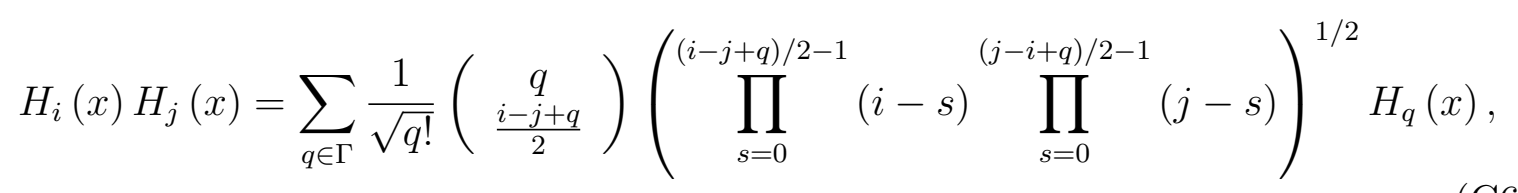

with

$$
\Gamma=\left\{q \in \mathbb{N}:|i-j| \leq q \leq i+j ; \quad \frac{i-j+q}{2} \in \mathbb{N}\right\}
$$

We can rewrite (C6) as

$$
\begin{aligned}
H_{i}(x) H_{j}(x) & =\sum_{q \in \Gamma} \frac{(i ! j ! q !)^{1 / 2}}{\left(\frac{i+j-q}{2}\right) !\left(\frac{i+q-j}{2}\right) !\left(\frac{q+j-i}{2}\right) !} H_{q}(x) \\
& =\sum_{q \in \Gamma} a_{i j, q} H_{q}(x)
\end{aligned}
$$

after verifying that $a_{i j, q}=a_{i q, j}=a_{j i, q}=a_{j q, i}=a_{q i, j}=a_{q j, i}$ by using some properties of the binomial coefficients. Hence, we will have that

$$
\sum_{i=0}^{m} \sum_{j=0}^{m} \frac{\nu_{i} \nu_{j}}{\boldsymbol{\nu}^{\prime} \boldsymbol{\nu}} H_{i}(x) H_{j}(x)=\sum_{i=0}^{m} \sum_{j=0}^{m} \sum_{k \in \Gamma} \frac{\nu_{i} \nu_{j}}{\boldsymbol{\nu}^{\prime} \boldsymbol{\nu}} a_{i, j, k} H_{k}(x) .
$$

Finally, if we equate (C5) and (C7), we obtain the desired result. 


\section{Proposition 2}

Consider the expanded SNP density function (4). Then

$$
\begin{aligned}
E_{f}\left[H_{k}(x)\right] & =\int_{-\infty}^{\infty} \phi(x) H_{k}(x)\left(\sum_{i=0}^{2 m} \gamma_{k}(\boldsymbol{\nu}) H_{i}(x)\right) d x \\
& =\sum_{i=0}^{2 m} \gamma_{k}(\boldsymbol{\nu}) E_{\phi}\left[H_{i}(x) H_{k}(x)\right]
\end{aligned}
$$

We can easily obtain (7) by using the property that $E_{\phi}\left[H_{i}(x) H_{k}(x)\right]=1$ if $i=k$ and zero otherwise.

\section{Lemma 1}

By using Proposition 2 we can directly obtain the matrices:

$$
A_{k}=\left(\begin{array}{ccc}
a_{00, k} & & \\
a_{10, k} & a_{11, k} & \\
a_{20, k} & a_{21, k} & a_{22, k}
\end{array}\right)
$$

for $k=1, \ldots, 4$ and $m=2$. Specifically,

$$
\begin{aligned}
A_{1}=\left(\begin{array}{ccc}
0 & & \\
1 & 0 & \\
0 & \sqrt{2} & 0
\end{array}\right) ; & A_{2}=\left(\begin{array}{ccc}
0 & & \\
0 & \sqrt{2} & \\
1 & 0 & 2 \sqrt{2}
\end{array}\right), \\
A_{3}=\left(\begin{array}{ccc}
0 & & \\
0 & 0 & \\
0 & \sqrt{3} & 0
\end{array}\right) ; & A_{4}=\left(\begin{array}{ccc}
0 & & \\
0 & 0 & \\
0 & 0 & \sqrt{6}
\end{array}\right) .
\end{aligned}
$$

On this basis, we can directly compute $E_{f}\left[H_{k}(x)\right]$ in (7). Finally, we can apply the equations in (6) to obtain the values of $\mu_{x}^{\prime}(k)$.

\section{Proposition 3}

Note that

$$
\begin{aligned}
E_{f}\left(e^{t x}\right) & =\sum_{k=0}^{2 m} \gamma_{k}(\boldsymbol{\nu}) \int_{-\infty}^{+\infty} e^{t x} H_{k}(x) \phi(x) d x \\
& =\sum_{k=0}^{2 m} \gamma_{k}(\boldsymbol{\nu}) E_{\phi}\left[e^{t x} H_{k}(x)\right]
\end{aligned}
$$

and that

$$
\int H_{k}(x) \phi(x) d x=\frac{-1}{\sqrt{k}} H_{k-1}(x) \phi(x) .
$$

If we consider (C9), and integrate by parts (C8), we obtain:

$$
\begin{aligned}
E_{\phi}\left[e^{t x} H_{k}(x)\right] & =\left[e^{t x} H_{k-1}(x) \phi(x) / \sqrt{k}\right]_{-\infty}^{+\infty}+\frac{t}{\sqrt{k}} E_{\phi}\left[e^{t x} H_{k-1}(x)\right] \\
& =\frac{t}{\sqrt{k}} E_{\phi}\left[e^{t x} H_{k-1}(x)\right] .
\end{aligned}
$$


where the subindex $\phi$ denotes integration with respect to the standard normal density. By l'Hospital rule, we can then verify that $e^{t x} H_{k-1}(x) \phi(x) \rightarrow 0 \forall k \geq 1$ when $x \rightarrow \pm \infty$. Hence,

$$
E_{\phi}\left[e^{t x} H_{k}(x)\right]=\frac{t^{k}}{\sqrt{k !}} e^{t^{2} / 2}
$$

In addition, given (C8) and (C10), we will have that:

$$
\begin{aligned}
E\left(e^{\lambda x}\right) & =e^{t^{2} / 2} \sum_{k=0}^{2 m} \gamma_{k}(\boldsymbol{\nu}) \frac{t^{k}}{\sqrt{k !}} \\
& =e^{\lambda^{2} / 2} \Lambda(\boldsymbol{\theta}, t) .
\end{aligned}
$$

On the other hand, the characteristic function can be written as

$$
\begin{aligned}
\psi_{s n p}(t) & =\int_{-\infty}^{+\infty} \exp (i t x) \phi(x) \sum_{j=0}^{2 m} \gamma_{j}(\boldsymbol{\nu}) H_{j}(x) d x \\
& =\sum_{j=0}^{2 m} \gamma_{j}(\boldsymbol{\nu}) \int_{-\infty}^{+\infty} \exp (i t x) \phi(x) H_{j}(x) d x
\end{aligned}
$$

where

$$
\int_{-\infty}^{+\infty} \exp (i t x) \phi(x) H_{0}(x) d x=\exp \left(\frac{-t^{2}}{2}\right)
$$

coincides with the characteristic function of a standard normal variable. Then, using integration by parts we will have that

$$
\begin{aligned}
\int_{-\infty}^{+\infty} \exp (i t x) \phi(x) H_{1}(x) d x & =-\exp (i t x) \phi(x)]_{-\infty}^{+\infty}+i t \int_{-\infty}^{+\infty} \exp (i t x) \phi(x) d x \\
& =i t \exp \left(\frac{-t^{2}}{2}\right) .
\end{aligned}
$$

Finally, we can combine the relationships in (2) with

$$
H_{k}^{\prime}(x)=\sqrt{k} H_{k-1}(x),
$$

to show by induction that

$$
\int_{-\infty}^{+\infty} \exp (i t x) \phi(x) H_{k}(x) d x=\frac{(i t)^{k}}{\sqrt{k !}} \exp \left(\frac{-t^{2}}{2}\right) .
$$

\section{Proposition 4}

Since $x_{k}$ are iid, we can use Proposition 3 to show that the characteristic function of $q$ can be expressed as

$$
\psi_{q}(t)=\prod_{k=1}^{n}\left[\exp \left(\frac{-p_{k}^{2} t^{2}}{2}\right) \sum_{j=0}^{2 m} \frac{\left(i p_{k} t\right)^{j}}{\sqrt{j !}} \gamma_{j}(\boldsymbol{\nu})\right]
$$


If we expand (C11), we will obtain:

$$
\psi_{q}(t)=\exp \left(\frac{-\|p\|^{2} t^{2}}{2}\right) \sum_{j=0}^{2 m n} \frac{(i t)^{j}}{\sqrt{j !}}\|p\|^{j} d_{j}(\boldsymbol{\nu}, \mathbf{p}),
$$

where the coefficients $d_{j}(\boldsymbol{\nu}, \mathbf{p})$ are such that

$$
\prod_{k=1}^{n}\left[\sum_{j=0}^{2 m} \frac{\gamma_{j}(\boldsymbol{\nu})}{\sqrt{j !}}\left(p_{k} z\right)^{j}\right]=\sum_{j=0}^{2 m n} \frac{d_{j}(\boldsymbol{\nu}, \mathbf{p})}{\sqrt{j !}} z^{j}
$$

for all $z$. Hence, from (C13), it is straightforward to obtain (12). Finally, we can use Proposition 3 to show that the characteristic function of $(11)$ is (C12), which proves that the density function of $q$ is indeed (11).

\section{Proposition 5}

Consider the generating function of Hermite polynomials (see Bontemps and Meddahi, 2005):

$$
\exp \left(z t-\frac{t^{2}}{2}\right)=\sum_{k=0}^{\infty} \frac{H_{k}(z)}{\sqrt{k !}} t^{k}
$$

Notice that, using both the relation $z=a+b x$ and (C14), we can write the generating function as

$$
\begin{aligned}
\exp \left(z t-\frac{t^{2}}{2}\right) & =\exp \left(\frac{b^{2} t^{2}}{2}\right) \exp \left(b t x-\frac{b^{2} t^{2}}{2}\right) \exp \left(a t-\frac{t^{2}}{2}\right) \\
& =\exp \left(\frac{b^{2} t^{2}}{2}\right)\left\{\sum_{s=0}^{\infty} \frac{H_{s}(x)}{\sqrt{s !}}(b t)^{s}\right\}\left\{\sum_{m=0}^{\infty} \frac{H_{m}(a)}{\sqrt{m !}} t^{m}\right\} .
\end{aligned}
$$

If we compute the expected value of the product of the generating function in $(\mathrm{C} 14)$ times the Hermite polynomial of order $i$, both with argument $x$, where $x$ is a standard normal variable, we get:

$$
E_{\phi}\left[\exp \left((a+b x) t-\frac{t^{2}}{2}\right) H_{i}(x)\right]=\sum_{k=0}^{\infty} \frac{E_{\phi}\left[H_{k}(a+b x) H_{i}(x)\right]}{\sqrt{k !}} t^{k} .
$$

Analogously, we can obtain from (C15) that

$$
\begin{aligned}
E_{\phi}\left[\exp \left((a+b x) t-\frac{t^{2}}{2}\right) H_{i}(x)\right]= & \exp \left(\frac{b^{2} t^{2}}{2}\right)\left\{\sum_{m=0}^{\infty} \frac{H_{m}(a)}{\sqrt{m !}} t^{m}\right\} \\
& \times\left\{\sum_{s=0}^{\infty} \frac{E_{\phi}\left[H_{s}(x) H_{i}(x)\right]}{\sqrt{s !}}(b t)^{s}\right\} .
\end{aligned}
$$

If we then combine the orthogonality property of the Hermite polynomials with the Taylor expansion for the above exponential function, we obtain

$$
\begin{aligned}
E_{\phi}\left[\exp \left((a+b x) t-\frac{t^{2}}{2}\right) H_{i}(x)\right] & =\frac{(b t)^{i}}{\sqrt{i !}} \exp \left(\frac{b^{2} t^{2}}{2}\right) \sum_{m=0}^{\infty} \frac{H_{m}(a)}{\sqrt{m !}} t^{m} \\
& =\frac{b^{i}}{\sqrt{i !}} \sum_{j=0}^{\infty} \sum_{m=0}^{\infty} \frac{H_{m}(a)}{j ! 2^{j} \sqrt{m !}} b^{2 j} t^{2 j+i+m}
\end{aligned}
$$


Finally, if we define $l=2 j+i+m$, we can write the above equation as

$$
E_{\phi}\left[\exp \left((a+b x) t-\frac{t^{2}}{2}\right) H_{i}(x)\right]=\frac{b^{i}}{\sqrt{i !}} \sum_{j=0}^{\infty} \sum_{l=i+2 j}^{\infty} \frac{H_{l-i-2 j}(a)}{j ! 2^{j} \sqrt{(l-i-2 j) !}} b^{2 j} t^{l} .
$$

Next, we can find the coefficients that multiply $t^{k}$ for $k=0,1,2, \cdots$, by comparing (C16) and (C17):

- When $i>k$ :

$$
E_{\phi}\left[H_{k}(a+b x) H_{i}(x)\right]=0 .
$$

- When $i=k$ :

$$
E_{\phi}\left[H_{i}(a+b x) H_{i}(x)\right]=b^{i} .
$$

- When $k>i$ and $k-i$ is an even number:

$$
E_{\phi}\left[H_{k}(a+b x) H_{i}(x)\right]=b^{i} \sqrt{\frac{k !}{i !}} \sum_{j=0}^{\frac{k-i}{2}} \frac{H_{k-i-2 j}(a)}{j ! \sqrt{(k-i-2 j) ! 2^{j}}} b^{2 j} .
$$

- When $k>i$ and $k-i$ is an odd number:

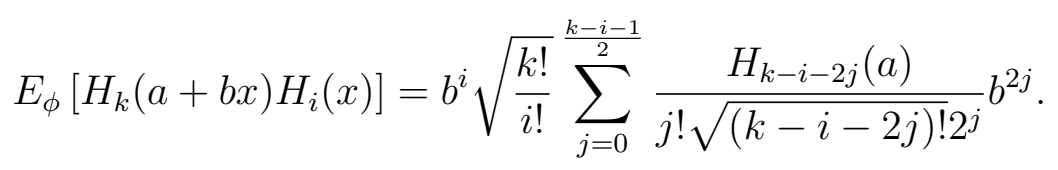

\section{Proposition 6}

Since we can write $y_{T}$ as $y_{T}=\delta_{\mathbb{P}}+\lambda_{\mathbb{P}} x^{\mathbb{P}}$, the arbitrage free conditions become

$$
\begin{aligned}
E_{\mathbb{P}}\left[\exp \left(\alpha \lambda_{\mathbb{P}} x^{\mathbb{P}}\right)\right] & =\exp \left[-\alpha \delta_{\mathbb{P}}-\beta \tau-r \tau\right], \\
E_{\mathbb{P}}\left[\exp \left((1+\alpha) \lambda_{\mathbb{P}} x^{\mathbb{P}}\right)\right] & =\exp \left[-(1+\alpha) \delta_{\mathbb{P}}-\beta \tau\right] .
\end{aligned}
$$

Then, using Proposition 3, we can easily obtain (22) and (23) from the previous two equations.

\section{Proposition 7}

Using (3) and (25) we can write

$$
\begin{aligned}
f^{\mathbb{Q}}\left(y_{T}\right)= & \exp (r \tau) \exp \left(\alpha y_{T}+\beta \tau\right) \\
& \times \frac{\phi\left(\frac{y_{T}-\delta_{\mathbb{P}}}{\lambda_{\mathbb{P}}}\right)}{\boldsymbol{\nu}^{\prime} \boldsymbol{\nu} \lambda_{\mathbb{P}}}\left[\sum_{i=0}^{m} \nu_{i} H_{i}\left(\frac{y_{T}-\delta_{\mathbb{P}}}{\lambda_{\mathbb{P}}}\right)\right]^{2} .
\end{aligned}
$$

We can rearrange the elements in $(\mathrm{C} 18)$ as

$$
\begin{aligned}
f^{\mathbb{Q}}\left(y_{T}\right)= & \exp (r \tau+\beta \tau) \exp \left(\alpha \delta_{\mathbb{P}}+\frac{\alpha^{2} \lambda_{\mathbb{P}}^{2}}{2}\right) \\
& \times \frac{\phi\left(\frac{y_{T}-\left(\delta_{\mathbb{P}}+\alpha \lambda_{\mathbb{P}}^{2}\right)}{\lambda_{\mathbb{P}}}\right)}{\boldsymbol{\nu}^{\prime} \boldsymbol{\nu} \lambda_{\mathbb{P}}}\left[\sum_{i=0}^{m} \nu_{i} H_{i}\left(\frac{y_{T}-\delta_{\mathbb{P}}}{\lambda_{\mathbb{P}}}\right)\right]^{2} \\
= & \frac{\phi\left(\frac{y_{T}-\delta_{\mathbb{Q}}}{\lambda_{\mathbb{Q}}}\right)}{\boldsymbol{\theta}^{\prime} \boldsymbol{\theta} \lambda_{\mathbb{Q}}}\left[\sum_{i=0}^{m} \theta_{i} H_{i}\left(\frac{y_{T}-\delta_{\mathbb{Q}}}{\lambda_{\mathbb{Q}}}\right)\right]^{2},
\end{aligned}
$$


where $\delta_{\mathbb{Q}}=\delta_{\mathbb{P}}+\alpha \lambda_{\mathbb{P}}^{2}, \lambda_{\mathbb{Q}}=\lambda_{\mathbb{P}}$. The parameters in the vector $\boldsymbol{\theta}=\left(\theta_{0}, \theta_{1}, \cdots \theta_{m}\right)$ can be easily obtained by noting that we can always rewrite (C19) in terms of a squared sum of Hermite polynomials in $\left(y_{T}-\delta_{\mathbb{Q}}\right) / \lambda_{\mathbb{Q}}$. That is, we can always find the value of $\boldsymbol{\theta}$ such that

$$
\sum_{i=0}^{m} \theta_{i} H_{i}\left(\frac{y_{T}-\delta_{\mathbb{Q}}}{\lambda_{\mathbb{Q}}}\right)=\sum_{i=0}^{m} \nu_{i} H_{i}\left(\frac{y_{T}-\delta_{\mathbb{P}}}{\lambda_{\mathbb{P}}}\right) .
$$

Starting from the right-hand side, we can write

$$
\sum_{i=0}^{m} \nu_{i} H_{i}\left(\frac{y_{T}-\delta_{\mathbb{P}}}{\lambda_{\mathbb{P}}}\right)=\sum_{i=0}^{m} \nu_{i} H_{i}\left(\frac{y_{T}-\delta_{\mathbb{Q}}}{\lambda_{\mathbb{Q}}}+\alpha \lambda_{\mathbb{P}}\right) .
$$

Then, using (B4), we can show that (C22) equals

$$
\sum_{k=0}^{m} \sum_{j=0}^{k} \nu_{k} \frac{1}{j !} \sqrt{\frac{k !}{(k-j) !}} H_{k-j}\left(\frac{y_{T}-\delta_{\mathbb{Q}}}{\lambda_{\mathbb{Q}}}\right)\left(\alpha \lambda_{\mathbb{P}}\right)^{j},
$$

which, through the change of indices $i=k-j$ becomes

$$
\sum_{k=0}^{m} \sum_{i=0}^{k} \nu_{k} \frac{1}{(k-i) !} \sqrt{\frac{k !}{i !}} H_{i}\left(\frac{y_{T}-\delta_{\mathbb{Q}}}{\lambda_{\mathbb{Q}}}\right)\left(\alpha \lambda_{\mathbb{P}}\right)^{k-i} .
$$

Now, if we compare (C23) with (C21), it is straightforward to find (29). Finally, we only need to check that the integrating constants are equal, i.e.

$$
\boldsymbol{\theta}^{\prime} \boldsymbol{\theta}=\boldsymbol{\nu}^{\prime} \boldsymbol{\nu} \exp \left(-r \tau-\beta \tau-\alpha \delta_{\mathbb{P}}-\frac{\alpha^{2} \lambda_{\mathbb{P}}^{2}}{2}\right)
$$

We have already shown that both (C19) and (C20) are proportional. Since both expressions are well defined densities in the sense that both integrate to one, (C24) must necessarily be satisfied. In consequence, $y_{T}$ can be written under the risk neutral measure as

$$
y_{T}=\delta_{\mathbb{Q}}+\lambda_{\mathbb{Q}} x_{T}^{\mathbb{Q}},
$$

where $x_{T}^{\mathbb{Q}}$ is a non-standardised SNP variable with parameters $\boldsymbol{\theta}$. Hence, both the real and the risk-neutral measures have a SNP distribution of the same order. In particular, if we express the asset price $S_{T}$ under the risk-neutral measure as in (26), where $\kappa_{T}=$ $a(\boldsymbol{\theta})+b(\boldsymbol{\theta}) x_{T}^{\mathbb{Q}}$, then we can easily relate the risk-neutral drift and volatility by the following relations

$$
\begin{gathered}
\left(\mu^{\mathbb{Q}}-\frac{\left(\sigma^{\mathbb{Q}}\right)^{2}}{2}\right) \tau+\sigma^{\mathbb{Q}} \sqrt{\tau} a(\boldsymbol{\theta})=\delta_{\mathbb{Q}}, \\
\sigma^{\mathbb{Q}} \sqrt{\tau} b(\boldsymbol{\theta})=\lambda_{\mathbb{Q}} .
\end{gathered}
$$

From (C27), it is straightforward to obtain (28), while the relationship for the drift can easily be found by replacing (28) in (C26). 


\section{Proposition 8}

Let us start with (27). As we know, (21) implies

$$
\begin{aligned}
1 & =E_{\mathbb{P}}\left[M_{t, T} \exp \left(y_{T}\right)\right] \\
& =\exp (-r \tau) E_{\mathbb{Q}}\left[\exp \left(y_{T}\right)\right] .
\end{aligned}
$$

Hence, since $y_{T}$ can be written as $(\mathrm{C} 25)$ in the risk neutral measure, we can use $(\mathrm{C} 10)$ to show that

$$
\exp \left(r \tau-\delta_{\mathbb{P}}-\alpha \lambda_{\mathbb{P}}^{2}-\frac{1}{2} \lambda_{\mathbb{P}}^{2}\right)=\Lambda\left(\boldsymbol{\theta}, \lambda_{\mathbb{Q}}\right)
$$

where $\Lambda\left(\boldsymbol{\theta}, \lambda_{\mathbb{Q}}\right)$ is given in (8). From (C28), we can write

$$
\alpha \sigma^{2} b^{2}(\boldsymbol{\nu})=r-\mu-\frac{\sigma^{2}}{2}\left(b^{2}(\boldsymbol{\nu})-1\right)-\frac{\sigma}{\sqrt{\tau}} a(\boldsymbol{\nu})-\log \Lambda\left(\boldsymbol{\theta}, \lambda_{\mathbb{Q}}\right),
$$

which, once substituted in (27), yields (30).

\section{Proposition 9}

Consider the general option formula (35) and equation (19), and express the set corresponding to $\left\{S_{T}>K\right\}$, denoted as $A$ for brevity, as $\{x>d\}$, where $d$ is given in Proposition 9. Then, (35) can be rewritten as

$$
C_{t}^{S N P}=S_{t} \operatorname{Pr}_{\mathbb{Q}_{1}}[x>d]-K e^{-r \tau} \operatorname{Pr}_{\mathbb{Q}}[x>d] .
$$

If we apply the limits of integration $+\infty$ and $d$ to the indefinite integral (C9), taking into account that $H_{k}(x) \phi(x) \rightarrow 0$ when $x \rightarrow+\infty$ (use L'Hospital rule), then

$$
\int_{d}^{\infty} H_{k}(x) \phi(x) d x=\frac{1}{\sqrt{k}} H_{k-1}(d) \phi(d), \quad k \geq 1 .
$$

Given (4), (C29) and the fact that $\gamma_{0}=1$, we can easily compute:

$$
\begin{aligned}
\operatorname{Pr}_{\mathbb{Q}}[x>d] & =\sum_{k=0}^{2 m} \gamma_{k}(\boldsymbol{\theta}) \int_{d}^{+\infty} H_{k}(x) \phi(x) d x \\
& =\Phi(-d)+\sum_{k=1}^{2 m} \frac{\gamma_{k}(\boldsymbol{\theta})}{\sqrt{k}} H_{k-1}(d) \phi(d) .
\end{aligned}
$$

Next, we will solve $E_{\mathbb{Q}_{1}}[\mathbf{1}(A)]=\operatorname{Pr}_{\mathbb{Q}_{1}}[x>d]$ by working under the $\mathbb{Q}$-measure, for which we must apply the Radon-Nikodym derivative, which in this case is just the inverse of (34), i.e.

$$
\frac{d \mathbb{Q}_{1}}{d \mathbb{Q}}=e^{-r \tau} \frac{S_{T}}{S_{t}}=e^{-r \tau+\delta_{\mathbb{Q}}+\lambda_{\mathbb{Q}} x} .
$$

Then,

$$
\begin{aligned}
E_{\mathbb{Q}_{1}}[\mathbf{1}(A)] & =E_{\mathbb{Q}}\left(\frac{d \mathbb{Q}_{1}}{d \mathbb{Q}} \mathbf{1}(A)\right) \\
& =e^{-r \tau+\delta_{\mathbb{Q}}} \sum_{k=0}^{2 m} \gamma_{k}(\boldsymbol{\theta}) \int_{d}^{\infty} e^{\lambda x} H_{k}(x) \phi(x) d x \\
& =e^{-r \tau+\delta_{\mathbb{Q}}} \sum_{k=0}^{2 m} \gamma_{k}(\boldsymbol{\theta}) E_{\phi}\left[e^{\lambda_{\mathbb{Q}} x} H_{k}(x) \mathbf{1}(A)\right] .
\end{aligned}
$$


For the sake of brevity, define $I_{k}^{*}$ as $E_{\phi}\left[e^{\lambda_{\circledast} x} H_{k}(x) \mathbf{1}(A)\right]$. The next step consists in computing $I_{k}^{*}$ for each $k$. When $k=0$, the integral is easy to obtain, namely, $I_{0}^{*}=$ $e^{\lambda_{\mathbb{Q}}^{2} / 2} \Phi(\lambda-d)$. But since $\gamma_{0}=1$, we can rewrite (C30) as

$$
\operatorname{Pr}_{\mathbb{Q}_{1}}[x>d]=e^{-r \tau+\delta_{\mathbb{Q}}}\left[e^{\lambda_{\mathbb{Q}}^{2} / 2} \Phi\left(\lambda_{\mathbb{Q}}-d\right)+\sum_{k=1}^{2 m} \gamma_{k}(\boldsymbol{\theta}) I_{k}^{*}\right] .
$$

Now, we will obtain the value of $I_{k}^{*}$ when $k \geq 1$. To do so, we will integrate by parts taking (C9) into account, which results in

$$
\begin{aligned}
I_{k}^{*} & =\int_{d}^{\infty} e^{\lambda_{\mathbb{Q}} x} H_{k}(x) \phi(x) d x \\
& =-\left[e^{\lambda_{\mathbb{Q}} x} H_{k-1}(x) \phi(x) / \sqrt{k}\right]_{d}^{\infty}+\frac{\lambda_{\mathbb{Q}}}{\sqrt{k}} \int_{d}^{\infty} e^{\lambda_{\mathbb{Q}} x} H_{k-1}(x) \phi(x) d x \\
& =-\left[e^{\lambda_{\mathbb{Q}} x} H_{k-1}(x) \phi(x) / \sqrt{k}\right]_{d}^{\infty}+\frac{\lambda_{\mathbb{Q}}}{\sqrt{k}} I_{k-1}^{*} .
\end{aligned}
$$

Since it is verified by applying L'Hospital rule that $e^{\lambda x} H_{k-1}(x) \phi(x) \rightarrow 0 \quad \forall k \geq 1$ when $x \rightarrow \infty$, then

$$
I_{k}^{*}=\frac{1}{\sqrt{k}} e^{\lambda_{\mathbb{Q}} d} H_{k-1}(d) \phi(d)+\frac{\lambda_{\mathbb{Q}}}{\sqrt{k}} I_{k-1}^{*} .
$$

Finally, we can recursively obtain the formula for $I_{k}^{*}$ given in (37).

\section{Proposition 10}

Since the roots of $P_{2 m}(x)$ are real and double or complex conjugates, we can express this polynomial as

$$
\begin{aligned}
P_{2 m}(x) & =\prod_{j=1}^{j=m}\left[\left(x-a_{j}\right)^{2}+b_{j}^{2}\right] \\
& =\prod_{j=1}^{j=m}\left[\left(x-a_{j}-i b_{j}\right)\left(x-a_{j}+i b_{j}\right)\right]
\end{aligned}
$$

Alternatively, we can write $P_{2 m}(x)$ as a sum of two squared polynomials of order $m$ :

$$
\begin{aligned}
P_{2 m}(x) & =\underbrace{\prod_{j=1}^{j=m}\left(x-a_{j}-i b_{j}\right)}_{Q(x)} \underbrace{\prod_{j=1}^{j=m}\left(x-a_{j}+i b_{j}\right)}_{\bar{Q}(x)} \\
& =\operatorname{Re}^{2}[Q(x)]+\operatorname{Im}^{2}[Q(x)]
\end{aligned}
$$

where $\bar{Q}(x)$ is the complex conjugate of $Q(x)$. Furthermore, it can be shown that the order of $\operatorname{Re}[Q(x)]=P_{1, m}(x)$ is $m$, while the order of $\operatorname{Im}[Q(x)]=P_{2, m-1}(x)$ is $m-1$ at most. Hence, we can express the GSNP as:

$$
f_{G S N P}\left(x ; \boldsymbol{\nu}_{1}, \boldsymbol{\nu}_{2}\right)=\phi(x)\left[P_{1, m}^{2}(x)+P_{2, m-1}^{2}(x)\right]
$$

where $P_{i, m_{i}}(x)=k_{i}\left[\nu_{i 0}+\nu_{i 1} H_{1}(x)+\cdots \nu_{i m_{i}} H_{m_{i}}(x)\right]$, for $i=1,2, m_{1}=m$ and $m_{2}=m-$ 1. Since this density is homogeneous of degree zero, we can chose $k_{1}=p\left(\boldsymbol{\nu}_{1}, \boldsymbol{\nu}_{2}\right) /\left(\boldsymbol{\nu}_{1}^{\prime} \boldsymbol{\nu}_{1}\right)$, and $k_{2}=\left[1-p\left(\boldsymbol{\nu}_{1}, \boldsymbol{\nu}_{2}\right)\right] /\left(\boldsymbol{\nu}_{1}^{\prime} \boldsymbol{\nu}_{1}\right)$ without lost of generality. 


\section{Proposition 11}

Given (26) for $S_{T}$ where $\kappa^{*}$ has a pdf defined in (15), and considering (17), we have that

$$
\begin{aligned}
g\left(\kappa^{*}\right) & =\phi\left(\kappa^{*}\right) \sum_{k=0}^{\infty} c_{k} H_{k}\left(\kappa^{*}\right) \\
& =\phi\left(\kappa^{*}\right)\left[1+\frac{s k}{\sqrt{3 !}} H_{3}\left(\kappa^{*}\right)+\frac{k u-3}{\sqrt{4 !}}+\sum_{k=5}^{\infty} c_{k} H_{k}\left(\kappa^{*}\right)\right] .
\end{aligned}
$$

Therefore, the call price $C_{t}^{S N P}$ can be rewritten as:

$$
\begin{aligned}
C_{t}^{S N P}= & \xi_{0}+\xi_{3} s k+\xi_{4}(k u-3)+\zeta \\
= & e^{-r \tau} \int_{\omega}^{\infty}\left(S_{T}\left(\kappa^{*}\right)-K\right) \phi\left(\kappa^{*}\right) d \kappa^{*} \\
& +\frac{s k}{\sqrt{3 !}} e^{-r \tau} \int_{\omega}^{\infty}\left(S_{T}\left(\kappa^{*}\right)-K\right) H_{3}\left(\kappa^{*}\right) \phi\left(\kappa^{*}\right) d \kappa^{*} \\
& +\frac{k u-3}{\sqrt{4 !}} e^{-r \tau} \int_{\omega}^{\infty}\left(S_{T}\left(\kappa^{*}\right)-K\right) H_{4}\left(\kappa^{*}\right) \phi\left(\kappa^{*}\right) d \kappa^{*} \\
& +e^{-r \tau} \sum_{k=5}^{\infty} c_{k} \int_{\omega}^{\infty}\left(S_{T}\left(\kappa^{*}\right)-K\right) H_{k}\left(\kappa^{*}\right) \phi\left(\kappa^{*}\right) d \kappa^{*},
\end{aligned}
$$

where $\omega$ is such that $S_{T}(\omega)=K$. Next, we will compute the values of $\xi$.

- For $\xi_{0}$ :

$$
\begin{aligned}
\xi_{0} & =e^{-r \tau} \int_{\omega}^{\infty}\left(S_{T}\left(\kappa^{*}\right)-K\right) \phi\left(\kappa^{*}\right) d \kappa^{*} \\
& =S_{t} e^{-r \tau+\bar{\mu}_{\tau}} \int_{\omega}^{\infty} e^{\sigma_{\tau} \kappa^{*}} \phi\left(\kappa^{*}\right) d \kappa^{*}-K e^{-r \tau} \Phi(-\omega) \\
& =S_{t} e^{\left(\mu^{\mathbb{Q}}-r\right) \tau} \Phi\left(d_{1}^{*}\right)-K e^{-r \tau} \Phi\left(d_{1}^{*}+\sigma_{\tau}\right),
\end{aligned}
$$

where $\bar{\mu}_{\tau}=\left(\mu^{\mathbb{Q}}-\sigma^{2} / 2\right) \tau$ and $d_{1}^{*}=\sigma_{\tau}-\omega$.

To obtain $\xi_{3}$ and $\xi_{4}$, we will use (37) and (C29). Specifically:

- For $\xi_{3}$ :

$$
\begin{gathered}
\xi_{3}=\frac{1}{\sqrt{3 !}} e^{-r \tau} \int_{\omega}^{\infty}\left(S_{T}\left(\kappa^{*}\right)-K\right) H_{3}\left(\kappa^{*}\right) \phi\left(\kappa^{*}\right) d \kappa^{*} \\
=\frac{1}{\sqrt{3 !}}\left\{S_{t} e^{-r \tau+\bar{\mu}_{\tau}} \int_{\omega}^{\infty} e^{\sigma_{\tau} \kappa^{*}} H_{3}\left(\kappa^{*}\right) \phi\left(\kappa^{*}\right) d \kappa^{*}-K e^{-r \tau} \int_{\omega}^{\infty} H_{3}\left(\kappa^{*}\right) \phi\left(\kappa^{*}\right) d \kappa^{*}\right\} \\
=\frac{1}{\sqrt{3 !}}\left\{S_{t} e^{-r \tau+\bar{\mu}_{\tau}} I_{3}^{*}\left(\sigma_{\tau}, \omega\right)-\frac{1}{\sqrt{3}} K e^{-r \tau} H_{2}(\omega) \phi(\omega)\right\}
\end{gathered}
$$

where $I_{3}^{*}\left(\sigma_{\tau}, \omega\right)$ denotes the value of $I_{k}^{*}$ for $k=3$ as a function on $\left(\sigma_{\tau}, \omega\right)$ instead of $(\lambda, d)$. Since

$$
e^{\sigma_{\tau} \omega}=\frac{K e^{-\bar{\mu}_{\tau}}}{S_{t}}
$$


then

$$
I_{3}^{*}\left(\sigma_{\tau}, \omega\right)=\frac{e^{\sigma_{\tau}^{2} / 2}}{\sqrt{3 !}}\left[\sigma_{\tau}^{3} \Phi\left(\sigma_{\tau}-\omega\right)+\frac{K e^{-\mu^{*} \tau}}{S_{t}} \phi(\omega) \sum_{j=0}^{2} \sqrt{j !} \sigma_{\tau}^{2-j} H_{j}(\omega)\right] .
$$

Plugging $I_{3}^{*}\left(\sigma_{\tau}, \omega\right)$ into equation (C32), we finally obtain

$$
\begin{aligned}
\xi_{3}= & \frac{e^{\left(\mu^{\mathbb{Q}}-r\right) \tau}}{3 !}\left[S_{t} \sigma_{\tau}^{3} \Phi\left(\sigma_{\tau}-\omega\right)+K e^{-\mu^{*} \tau} \phi(\omega) \sum_{j=0}^{2} \sqrt{j !} \sigma_{\tau}^{2-j} H_{j}(\omega)\right] \\
& -\frac{1}{\sqrt{3 !}} \frac{1}{\sqrt{3}} K e^{-r \tau} H_{2}(\omega) \phi(\omega) \\
= & \frac{e^{\left(\mu^{\mathbb{Q}}-r\right) \tau}}{3 !} S_{t} \sigma_{\tau}^{3} \Phi\left(\sigma_{\tau}-\omega\right)+\frac{K}{3 !} e^{-r \tau} \phi(\omega)\left[\sigma_{\tau}^{2}+\sigma_{\tau} \omega\right] .
\end{aligned}
$$

Following the same idea as Jurczenko, Maillet, and Negrea (2002a), we can write:

$$
\left(\sigma_{\tau}-\omega\right)^{2}=\omega^{2}+2 \log \left(S_{t} e^{\mu^{*} \tau} / K\right)
$$

so that

$$
\phi\left(\sigma_{\tau}-\omega\right)=\left(K / S_{t} e^{\mu^{*} \tau}\right) \phi(\omega),
$$

which implies that

$$
K \phi(\omega)=S_{t} e^{\mu^{*} \tau} \phi\left(\sigma_{\tau}-\omega\right) .
$$

If we substitute the above equation into (C33), we obtain:

$$
\begin{aligned}
\xi_{3} & =\frac{\sigma_{\tau}}{3 !} S_{t} e^{\left(\mu^{*}-r\right) \tau}\left[\sigma_{\tau}^{2} \Phi\left(\sigma_{\tau}-\omega\right)+\left(\sigma_{\tau}+\omega\right) \phi\left(\sigma_{\tau}-\omega\right)\right] \\
& =\frac{\sigma_{\tau}}{3 !} S_{t} e^{\left(\mu^{*}-r\right) \tau}\left[\sigma_{\tau}^{2} \Phi\left(d_{1}^{*}\right)+\left(2 \sigma_{\tau}-d_{1}^{*}\right) \phi\left(d_{1}^{*}\right)\right]
\end{aligned}
$$

- For $\xi_{4}$ :

$$
\begin{aligned}
\xi_{4} & =\frac{1}{\sqrt{4 !}} e^{-r \tau} \int_{\omega}^{\infty}\left(S_{T}\left(\kappa^{*}\right)-K\right) H_{4}\left(\kappa^{*}\right) \phi\left(\kappa^{*}\right) d \kappa^{*} \\
& =\frac{1}{\sqrt{4 !}}\left\{S_{t} e^{-r \tau+\bar{\mu}_{\tau}} I_{4}^{*}\left(\sigma_{\tau}, \omega\right)-\frac{1}{\sqrt{4}} K e^{-r \tau} H_{3}(\omega) \phi(\omega)\right\} .
\end{aligned}
$$

Following the same procedure as in $\xi_{3}$, we can show that:

$$
\xi_{4}=\frac{\sigma_{\tau}}{4 !} S_{t} e^{\left(\mu^{*}-r\right) \tau}\left[\sigma_{\tau}^{3} \Phi\left(d_{1}^{*}\right)+\left(3 \sigma_{\tau}^{2}-3 d_{1}^{*} \sigma_{\tau}+d_{1}^{* 2}-1\right) \phi\left(d_{1}^{*}\right)\right] .
$$

\section{Lemma 2}

From (30), we have

BANCO de ESPAÑA 59 DOCUMENTO de tRABAJO N. 0707

$$
\mu^{\mathbb{Q}}=r-\frac{1}{\tau} \log \left[\exp \left(\sigma_{\tau} a(\boldsymbol{\theta})+\frac{1}{2} \sigma_{\tau}^{2}\left(b^{2}(\boldsymbol{\theta})-1\right)\right) \sum_{k=0}^{2 m} \gamma_{k}(\boldsymbol{\theta}) \frac{\left(\sigma_{\tau} b(\boldsymbol{\theta})\right)^{k}}{\sqrt{k !}}\right],
$$


where

$$
\begin{gathered}
\exp \left(\sigma_{\tau} a(\boldsymbol{\theta})+\frac{1}{2} \sigma_{\tau}^{2}\left(b^{2}(\boldsymbol{\theta})-1\right)\right)=1+a(\boldsymbol{\theta}) \sigma_{\tau}+\frac{a^{2}(\boldsymbol{\theta})+b^{2}(\boldsymbol{\theta})-1}{2} \sigma_{\tau}^{2} \\
+\frac{a^{3}(\boldsymbol{\theta})+3 a(\boldsymbol{\theta}) b^{2}(\boldsymbol{\theta})-3 a(\boldsymbol{\theta})}{6} \sigma_{\tau}^{3} \\
+\frac{3 b^{4}(\boldsymbol{\theta})-6 b^{2}(\boldsymbol{\theta})+3+6 a^{2}(\boldsymbol{\theta}) b^{2}(\boldsymbol{\theta})-6 a^{2}(\boldsymbol{\theta})+a^{4}(\boldsymbol{\theta})}{24} \sigma_{\tau}^{4} \\
+o\left(\sigma_{\tau}^{4}\right) .
\end{gathered}
$$

Then, from Proposition 1 we obtain that

$$
\begin{aligned}
\gamma_{0}(\boldsymbol{\theta})= & 1, \\
\gamma_{1}(\boldsymbol{\theta})= & \mu_{x}^{\prime}(1)=\frac{-a(\boldsymbol{\theta})}{b(\boldsymbol{\theta})}, \\
\gamma_{2}(\boldsymbol{\theta})= & \frac{\mu_{x}^{\prime}(2)-1}{\sqrt{2}}=\frac{a^{2}(\boldsymbol{\theta})-b^{2}(\boldsymbol{\theta})+1}{b^{2}(\boldsymbol{\theta}) \sqrt{2}}, \\
\gamma_{3}(\boldsymbol{\theta})= & \frac{\mu_{x}^{\prime}(3)-3 \mu_{x}^{\prime}(1)}{\sqrt{3 !}} \\
= & \frac{s k(\boldsymbol{\theta})-a^{3}(\boldsymbol{\theta})-3 a(\boldsymbol{\theta})+3 a(\boldsymbol{\theta}) b^{2}(\boldsymbol{\theta})}{b^{3}(\boldsymbol{\theta}) \sqrt{3 !}} \\
\gamma_{4}(\boldsymbol{\theta})= & \frac{\mu_{x}^{\prime}(4)-6 \mu_{x}^{\prime}(2)+3}{\sqrt{4 !}} \\
= & \frac{6 a^{2}(\boldsymbol{\theta})-6 a^{2}(\boldsymbol{\theta}) b^{2}(\boldsymbol{\theta})-6 b^{2}(\boldsymbol{\theta})+3 b^{4}(\boldsymbol{\theta})+3}{b^{4}(\boldsymbol{\theta}) \sqrt{4 !}} \\
& +\frac{6 a^{2}(\boldsymbol{\theta})-6 a^{2}(\boldsymbol{\theta}) b^{2}(\boldsymbol{\theta})-6 b^{2}(\boldsymbol{\theta})+3 b^{4}(\boldsymbol{\theta})+3}{b^{4}(\boldsymbol{\theta}) \sqrt{4 !}}
\end{aligned}
$$

Next, if we use the property that $o\left(n^{p}\right) o\left(n^{q}\right)=o\left(n^{p+q}\right)$ (see Davidson and MacKinnon, 1993), we will have

$$
\begin{gathered}
\exp \left(\sigma_{\tau} a(\boldsymbol{\theta})+\frac{1}{2} \sigma_{\tau}^{2}\left(b^{2}(\boldsymbol{\theta})-1\right)\right) \underbrace{\sum_{k=0}^{2 m} \gamma_{k}(\boldsymbol{\theta}) \frac{\left(\sigma_{\tau} b(\boldsymbol{\theta})\right)^{k}}{\sqrt{k !}}}_{o\left(\sigma_{\tau}^{0}\right)}=\left[\sum_{k=0}^{4} \gamma_{k}(\boldsymbol{\theta}) \frac{\left(\sigma_{\tau} b(\boldsymbol{\theta})\right)^{k}}{\sqrt{k !}}\right] \\
\times\left[1+a(\boldsymbol{\theta}) \sigma_{\tau}+\frac{a^{2}(\boldsymbol{\theta})+b^{2}(\boldsymbol{\theta})-1}{2} \sigma_{\tau}^{2}+\frac{a^{3}(\boldsymbol{\theta})+3 a(\boldsymbol{\theta}) b^{2}(\boldsymbol{\theta})-3 a(\boldsymbol{\theta})}{6} \sigma_{\tau}^{3}\right. \\
\left.+\frac{3 b^{4}(\boldsymbol{\theta})-6 b^{2}(\boldsymbol{\theta})+3+6 a^{2}(\boldsymbol{\theta}) b^{2}(\boldsymbol{\theta})-6 a^{2}(\boldsymbol{\theta})+a^{4}(\boldsymbol{\theta})}{24} \sigma_{\tau}^{4}\right]+o\left(\sigma_{\tau}^{4}\right) .
\end{gathered}
$$

Finally, we can use tedious but otherwise straightforward algebraic operations to show that a Taylor expansion of the argument in the logarithm of (C34) around $\sigma_{\tau}=0$ yields the proposed result. 


\section{Proposition 12}

We can rewrite $C_{t}^{S N P}$ in Proposition 11 as

$$
\begin{aligned}
C_{t}^{S N P}= & S_{t} e^{\left(\mu^{\mathbb{Q}}-r\right) \tau} \Phi\left(d_{1}^{*}\right)\left[1+\frac{s k}{3 !} \sigma_{\tau}^{3}+\frac{(k u-3)}{4 !} \sigma_{\tau}^{4}\right]-K e^{-r \tau} \Phi\left(d_{1}^{*}-\sigma_{\tau}\right) \\
& +\frac{s k}{3 !} \sigma_{\tau} S_{t} e^{\left(\mu^{\mathbb{Q}}-r\right) \tau}\left(2 \sigma_{\tau}-d_{1}^{*}\right) \phi\left(d_{1}^{*}\right) \\
& +\frac{(k u-3)}{4 !} \sigma_{\tau} S_{t} e^{\left(\mu^{\mathbb{Q}}-r\right) \tau}\left(3 \sigma_{\tau}^{2}-3 d_{1}^{*} \sigma_{\tau}+d_{1}^{* 2}-1\right) \phi\left(d_{1}^{*}\right),
\end{aligned}
$$

where we have neglected $\zeta$. From lemma 2, we finally have that

$$
\begin{aligned}
\exp \left[\left(\mu^{\mathbb{Q}}-r\right) \tau\right] & =\frac{1}{1+\frac{s k}{3 !} \sigma_{\tau}^{3}+\frac{(k u-3)}{4 !} \sigma_{\tau}^{4}+o\left(\sigma_{\tau}^{4}\right)} \\
& =\frac{1}{1+\frac{s k}{3 !} \sigma_{\tau}^{3}+\frac{(k u-3)}{4 !} \sigma_{\tau}^{4}}+o\left(\sigma_{\tau}^{4}\right)
\end{aligned}
$$

because as $o\left(n^{0}\right)+o\left(n^{p}\right)=o\left(n^{0}\right)$ (see Davidson and MacKinnon, 1993), which, substituted into (C35), gives

$$
\begin{aligned}
C_{t}^{S N P}= & S_{t} \Phi\left(d_{1}^{*}\right)-K e^{-r \tau} \Phi\left(d_{1}^{*}-\sigma_{\tau}\right) \\
& +\frac{s k}{3 !} \sigma_{\tau} S_{t} \frac{\left(2 \sigma_{\tau}-d_{1}^{*}\right) \phi\left(d_{1}^{*}\right)}{1+\frac{s k}{3 !} \sigma_{\tau}^{3}+\frac{(k u-3)}{4 !} \sigma_{\tau}^{4}} \\
& +\frac{(k u-3)}{4 !} \sigma_{\tau} S_{t} \frac{\left(3 \sigma_{\tau}^{2}-3 d_{1}^{*} \sigma_{\tau}+d_{1}^{* 2}-1\right) \phi\left(d_{1}^{*}\right)}{1+\frac{s k}{3 !} \sigma_{\tau}^{3}+\frac{(k u-3)}{4 !} \sigma_{\tau}^{4} .}
\end{aligned}
$$

Then, using again lemma 2, we can obtain the relationship

$$
d_{1}^{*}=d^{*}+o\left(\sigma_{\tau}^{4}\right)
$$

which, once introduced in (C36), yields the Corrado-Su modified formula after neglecting the terms $o\left(\sigma_{\tau}^{4}\right)$.

\section{Proposition 13}

Expanding $d_{1}^{*}$ around $d_{1}$, we have

$$
\begin{aligned}
d_{1}^{*}= & d_{1}-\frac{1}{\sigma \sqrt{\tau}} \log \left(1+\frac{s k}{3 !} \sigma_{\tau}^{3}+\frac{(k u-3)}{4 !} \sigma_{\tau}^{4}+o\left(\sigma_{\tau}^{4}\right)\right) \\
= & d_{1}-\frac{s k}{3 !} \sigma_{\tau}^{2}-\frac{(k u-3)}{4 !} \sigma_{\tau}^{3}+o\left(\sigma_{\tau}^{3}\right) \\
\Phi\left(d_{1}^{*}\right) & =\Phi\left(d_{1}\right)-\phi\left(d_{1}\right) \frac{s k}{3 !} \sigma_{\tau}^{2}+o\left(\sigma_{\tau}^{2}\right) \\
\Phi\left(d_{1}^{*}-\sigma_{\tau}\right) & =\Phi\left(d_{1}-\sigma_{\tau}\right)-\phi\left(d_{1}-\sigma_{\tau}\right) \frac{s k}{3 !} \sigma_{\tau}^{2}+o\left(\sigma_{\tau}^{2}\right) \\
& =\Phi\left(d_{1}-\sigma_{\tau}\right)-\phi\left(d_{1}\right) \frac{s k}{3 !} \sigma_{\tau}^{2}+o\left(\sigma_{\tau}^{2}\right)
\end{aligned}
$$




$$
\begin{aligned}
\frac{s k}{3 !} \sigma_{\tau} S_{t} \frac{\left(2 \sigma_{\tau}-d_{1}^{*}\right) \phi\left(d_{1}^{*}\right)}{1+\frac{s k}{3 !} \sigma_{\tau}^{3}+\frac{(k u-3)}{4 !} \sigma_{\tau}^{4}} & =\frac{s k}{3 !} \frac{\sigma_{\tau} S_{t}\left(2 \sigma_{\tau}-d_{1}\right) \phi\left(d_{1}\right)+o\left(\sigma_{\tau}^{2}\right)}{1+o\left(\sigma_{\tau}^{2}\right)} \\
& =\frac{s k}{3 !} \sigma_{\tau} S_{t}\left(2 \sigma_{\tau}-d_{1}\right) \phi\left(d_{1}\right)+o\left(\sigma_{\tau}^{2}\right)
\end{aligned}
$$

and

$$
\frac{(k u-3)}{4 !} \sigma_{\tau} S_{t} \frac{\left(3 \sigma_{\tau}^{2}-3 d_{1}^{*} \sigma_{\tau}+d_{1}^{* 2}-1\right) \phi\left(d_{1}^{*}\right)}{1+\frac{s k}{3 !} \sigma_{\tau}^{3}+\frac{(k u-3)}{4 !} \sigma_{\tau}^{4}}=\frac{(k u-3)}{4 !} \sigma_{\tau} S_{t}\left(d_{1}^{2}-3 d_{1} \sigma_{\tau}-1\right) \phi\left(d_{1}\right) .
$$

Then, we can easily take a Taylor series expansion of (C36) around $\sigma_{\tau}=0$. If we only retain the terms in $\sigma_{\tau}^{k}$, for $k=0,1,2$, we finally obtain the desired result.

\section{Proposition 14}

$\Psi$ is the implied volatility that equates the call market price $C_{t}$ to the Black-Scholes formula, i.e. $C_{t}=C_{t}^{B S}(\Psi)$ where $C_{t}^{B S}($.$) is the Black-Scholes formula. Following$ Jurczenko, Maillet, and Negrea (2002a), we can take a linear approximation of the BlackScholes formula around the true volatility $\sigma_{\tau}$ of the underlying asset

$$
C_{t}=C_{t}^{B S}(\Psi)=C_{t}^{B S}\left(\sigma_{\tau}\right)+\left.\frac{\partial C_{t}^{B S}(x)}{\partial x}\right|_{x=\sigma_{\tau}}\left(\Psi-\sigma_{\tau}\right)
$$

Since

$$
\left.\frac{\partial C_{t}^{B S}(x)}{\partial x}\right|_{x=\sigma_{\tau}}=K \phi\left[d_{1}\left(\sigma_{\tau}\right)-\sigma_{\tau}\right]=S_{t} e^{r \tau} \phi\left[d_{1}\left(\sigma_{\tau}\right)\right]
$$

then

$$
C_{t} \simeq C_{t}^{B S}\left(\sigma_{\tau}\right)+S_{t} \phi\left[d_{1}\left(\sigma_{\tau}\right)\right]\left(\Psi-\sigma_{\tau}\right)
$$

Finally, if the call market price follows the SNP model, i.e. $C_{t}=C_{t}^{S N P}$, we can equate (A2) and (C37) to obtain the approximation to $\Psi$ given in (A3). 


\section{BANCO DE ESPAÑA PUBLICATIONS}

\section{WORKING PAPERS ${ }^{1}$}

0601 ARTURO GALINDO, ALEJANDRO IZQUIERDO AND JOSÉ MANUEL MONTERO: Real exchange rates, dollarization and industrial employment in Latin America.

0602 JUAN A. ROJAS AND CARLOS URRUTIA: Social security reform with uninsurable income risk and endogenous borrowing constraints.

0603 CRISTINA BARCELÓ: Housing tenure and labour mobility: a comparison across European countries.

0604 FRANCISCO DE CASTRO AND PABLO HERNÁNDEZ DE COS: The economic effects of exogenous fiscal shocks in Spain: a SVAR approach.

0605 RICARDO GIMENO AND CARMEN MARTÍNEZ-CARRASCAL: The interaction between house prices and loans for house purchase. The Spanish case.

0606 JAVIER DELGADO, VICENTE SALAS AND JESÚS SAURINA: The joint size and ownership specialization in banks' lending.

0607 ÓSCAR J. ARCE: Speculative hyperinflations: When can we rule them out?

0608 PALOMA LÓPEZ-GARCÍA AND SERGIO PUENTE: Business demography in Spain: determinants of firm survival.

0609 JUAN AYUSO AND FERNANDO RESTOY: House prices and rents in Spain: Does the discount factor matter?

0610 ÓSCAR J. ARCE AND J. DAVID LÓPEZ-SALIDO: House prices, rents, and interest rates under collateral constraints.

0611 ENRIQUE ALBEROLA AND JOSÉ MANUEL MONTERO: Debt sustainability and procyclical fiscal policies in Latin America.

0612 GABRIEL JIMÉNEZ, VICENTE SALAS AND JESÚS SAURINA: Credit market competition, collateral and firms' finance.

0613 ÁNGEL GAVILÁN: Wage inequality, segregation by skill and the price of capital in an assignment model.

0614 DANIEL PÉREZ, VICENTE SALAS AND JESÚS SAURINA: Earnings and capital management in alternative loan loss provision regulatory regimes.

0615 MARIO IZQUIERDO AND AITOR LACUESTA: Wage inequality in Spain: Recent developments.

0616 K. C. FUNG, ALICIA GARCÍA-HERRERO, HITOMI IIAKA AND ALAN SUI: Hard or soft? Institutional reforms and infraestructure spending as determinants of foreign direct investment in China.

0617 JAVIER DÍAZ-CASSOU, ALICIA GARCÍA-HERRERO AND LUIS MOLINA: What kind of capital flows does the IMF catalyze and when?

0618 SERGIO PUENTE: Dynamic stability in repeated games.

0619 FEDERICO RAVENNA: Vector autoregressions and reduced form representations of DSGE models.

0620 AITOR LACUESTA: Emigration and human capital: Who leaves, who comes back and what difference does it make?

0621 ENRIQUE ALBEROLA AND RODRIGO CÉSAR SALVADO: Banks, remittances and financial deepening in receiving countries. A model.

0622 SONIA RUANO-PARDO AND VICENTE SALAS-FUMÁS: Morosidad de la deuda empresarial bancaria en España, 1992-2003. Modelos de la probabilidad de entrar en mora, del volumen de deuda en mora y del total de deuda bancaria, a partir de datos individuales de empresa.

0623 JUAN AYUSO AND JORGE MARTíNEZ: Assessing banking competition: an application to the Spanish market for (quality-changing) deposits.

0624 IGNACIO HERNANDO AND MARÍA J. NIETO: Is the Internet delivery channel changing banks' performance? The case of Spanish banks.

0625 JUAN F. JIMENO, ESTHER MORAL AND LORENA SAIZ: Structural breaks in labor productivity growth: The United States vs. the European Union.

0626 CRISTINA BARCELÓ: A Q-model of labour demand.

0627 JOSEP M. VILARRUBIA: Neighborhood effects in economic growth.

0628 NUNO MARTINS AND ERNESTO VILLANUEVA: Does limited access to mortgage debt explain why young adults live with their parents?

0629 LUIS J. ÁLVAREZ AND IGNACIO HERNANDO: Competition and price adjustment in the euro area.

0630 FRANCISCO ALONSO, ROBERTO BLANCO AND GONZALO RUBIO: Option-implied preferences adjustments, density forecasts, and the equity risk premium.

1. Previously published Working Papers are listed in the Banco de España publications catalogue. 
0631 JAVIER ANDRÉS, PABLO BURRIEL AND ÁNGEL ESTRADA: BEMOD: A dsge model for the Spanish economy and the rest of the Euro area.

0632 JAMES COSTAIN AND MARCEL JANSEN: Employment fluctuations with downward wage rigidity: The role of moral hazard.

0633 RUBÉN SEGURA-CAYUELA: Inefficient policies, inefficient institutions and trade.

0634 RICARDO GIMENO AND JUAN M. NAVE: Genetic algorithm estimation of interest rate term structure.

0635 JOSÉ MANUEL CAMPA, JOSÉ M. GONZÁLEZ-MÍNGUEZ AND MARÍA SEBASTIÁ-BARRIEL: Non-linear adjustment of import prices in the European Union.

0636 AITOR ERCE-DOMÍNGUEZ: Using standstills to manage sovereign debt crises.

0637 ANTON NAKOV: Optimal and simple monetary policy rules with zero floor on the nominal interest rate.

0638 JOSÉ MANUEL CAMPA AND ÁNGEL GAVILÁN: Current accounts in the euro area: An intertemporal approach.

0639 FRANCISCO ALONSO, SANTIAGO FORTE AND JOSÉ MANUEL MARQUÉS: Implied default barrier in credit default swap premia. (The Spanish original of this publication has the same number.)

0701 PRAVEEN KUJAL AND JUAN RUIZ: Cost effectiveness of R\&D and strategic trade policy.

0702 MARÍA J. NIETO AND LARRY D. WALL: Preconditions for a successful implementation of supervisors' prompt corrective action: Is there a case for a banking standard in the EU?

0703 PHILIP VERMEULEN, DANIEL DIAS, MAARTEN DOSSCHE, ERWAN GAUTIER, IGNACIO HERNANDO, ROBERTO SABBATINI AND HARALD STAHL: Price setting in the euro area: Some stylised facts from individual producer price data.

0704 ROBERTO BLANCO AND FERNANDO RESTOY: Have real interest rates really fallen that much in Spain?

0705 OLYMPIA BOVER AND JUAN F. JIMENO: House prices and employment reallocation: International evidence.

0706 ENRIQUE ALBEROLA AND JOSE M. ${ }^{a}$ SERENA: Global financial integration, monetary policy and reserve accumulation. Assessing the limits in emerging economies.

0707 ÁNGEL LEÓN, JAVIER MENCÍA AND ENRIQUE SENTANA: Parametric properties of semi-nonparametric distributions, with applications to option valuation

\begin{tabular}{|r|c|}
\hline & Unidad de Publicaciones \\
BANCODEESPAÑ & Alcalá, 522; 28027 Madrid \\
Eurosistema & Teléfono +34 913386363. Fax +34 913386488 \\
& Correo electrónico: publicaciones@bde.es \\
& www.bde.es \\
\hline
\end{tabular}

